\title{
An upwind vertex centred Finite Volume solver for Lagrangian solid dynamics
}

\author{
Miquel Aguirre, Antonio J. Gil ${ }^{1}$, Javier Bonet, Chun Hean Lee \\ Zienkiewicz Centre for Computational Engineering, College of Engineering \\ Swansea University, Singleton Park, SA2 8PP, United Kingdom
}

\begin{abstract}
A vertex centred Jameson-Schmidt-Turkel (JST) finite volume algorithm was recently introduced by the authors (Aguirre et al., 2014 [1]) in the context of fast solid isothermal dynamics. The spatial discretisation scheme was constructed upon a Lagrangian two-field mixed (linear momentum and the deformation gradient) formulation presented as a system of conservation laws [2-4]. In this paper, the formulation is further enhanced by introducing a novel upwind vertex centred finite volume algorithm with three key novelties. First, a conservation law for the volume map is incorporated into the existing two-field system to extend the range of applications towards the incompressibility limit (Gil et al., 2014 [5]). Second, the use of a linearised Riemann solver and reconstruction limiters is derived for the stabilisation of the scheme together with an efficient edge-based implementation. Third, the treatment of thermo-mechanical processes through a Mie-Grüneisen equation of state is incorporated in the proposed formulation. For completeness, the study of the eigenvalue structure of the resulting system of conservation laws is carried out to demonstrate hyperbolicity and obtain the correct time step bounds for non-isothermal processes. A series of numerical examples are presented in order to assess the robustness of the proposed methodology. The overall scheme shows excellent behaviour in shock and bending dominated nearly incompressible scenarios without spurious pressure oscillations, yielding second order of convergence for both velocities and stresses.
\end{abstract}

Keywords: Fast dynamics, Mie-Grüneisen, Finite Volume Method, Riemann Solver, Incompressible, Locking, Shock Capturing

\footnotetext{
${ }^{1}$ Corresponding author: a.j.gil@swansea.ac.uk
} 


\section{Introduction}

In practical engineering applications involving extremely complex geometries, meshing typically represents a large portion of the overall design and analysis time. In the computational mechanics community, the ability to perform calculations on tetrahedral meshes has become increasingly important. For these reasons, the automated tetrahedral mesh generators by means of Delaunay and advancing front techniques [6] have recently received increasing attention in a number of important application areas, such as cardiovascular tissue modelling [7], crash impact simulation [8], blast and fracture mechanics and complex multi-physics problems [9-12].

Unfortunately, modern tetrahedral element technology in solid mechanics (e.g. ANSYS AUTODYN, LS-DYNA, ABAQUS/Explicit, Altair HyperCrash), typically based on the use of the traditional Finite Element based second order displacement formulation $[13,14]$, possesses several distinct disadvantages, namely: (1) Reduced order of convergence for strains and stresses $[15,16] ;$ (2) High frequency noise in the vicinity of shocks [17-20]; (3) Stability issues associated to shear locking, volumetric locking [21] and pressure checkerboard instabilities [22].

To address the shortcomings mentioned above, a wide variety of enhanced discretisation technologies have been developed. As an example, for the case of nearly incompressible materials, the mean dilatational hexahedral formulation [23-25] where constant interpolation is used for the calculation of volumetric stresses [26] has attracted industrial interest, as the modifications associated to the classical displacement based formulation are minor. High order elements [27-29] (also known as p-refinement) can alternatively be used. However, the increase in the number of integration points can drastically reduce the computational efficiency of these schemes in comparison with low order approaches [30], specially when either complex constitutive laws (i.e. anisotropic visco-elastic models are often used in the medical field [31]) or contact surfaces [32], or a combination of both, must be modelled.

The success of nodally integrated tetrahedral elements was first reported in [33], where the volumetric strain energy functional was approximated through averaged nodal pressures. Extensive efforts have since been made to further develop this class of averaged nodal strain technologies with the use of various types of stabilisation [34-39]. Despite exhibiting geometric 
locking-free behaviours, the resulting formulation still suffers from spurious hydrostatic pressure fluctuations when simulating nearly incompressible materials.

Several authors have presented an alternative $\boldsymbol{p}$ - $\boldsymbol{F}$ mixed formulations in both Lagrangian solid and gas dynamics [1-4, 40-42]. Specifically, in references $[1,3,40,41]$, the authors presented a mixed conservation law for applications in Lagrangian fast solid dynamics, which are spatially discretised using tailor-made CFD technology. The use of a mixed approach proved to be very efficient in large strain solid dynamics, circumventing the above-mentioned drawbacks for the traditional displacement based techniques. Early attempts at applying CFD-like numerical techniques in the context of displacement based computational solid dynamics are reported in references [2, 43-48]. Eulerian Finite Volume Godunov methods, typically used for modelling compressible gas dynamics, were employed to model plastic flows in solid dynamics [49-51]. Furthermore, this methodology was also adapted to a Lagrangian framework by several authors [52, 53], but restricted to two dimensions.

The use of total Lagrangian description of the motion has clear advantages in the context of solid dynamics. Firstly, all the calculations are carried out based on undeformed mesh leading to a simple algorithm. Secondly, the Lagrangian settting follows the evolution of any material particle, which is of paramount importance in history dependent constitutive laws. Finally, the imposition of free surface boundary conditions is straightforward. On the contrary, the accuracy of the scheme would be adversely affected when experiencing very large deformations. This can be circumvented by employing adaptive remeshing techniques.

More recently, the $\boldsymbol{p}-\boldsymbol{F}$ formulation was improved in [5] for the case of nearly incompressible materials, by means of an additional conservation law for the Jacobian of the deformation $J$ (widely known as volume map conservation law [42, 54-57]), providing extra flexibility for the calculation of the volumetric stress. This innovative idea extended the range of use of the formulation to nearly and fully incompressible media. Moreover, further enhancement of the framework has recently been reported by the authors [58], when considering materials governed by a polyconvex constitutive law [59], enabling the symmetrisation of the resulting hyperbolic system of equations.

In this paper, the mixed $\boldsymbol{p}-\boldsymbol{F}-J$ is discretised via an adapted upwind vertex centred Finite Volume Method (FVM) for linear tetrahedral meshes [60]. One clear advantage of using the upwind method is the ability to introduce 
physically-based numerical dissipation into the formulation derived from the Rankine-Hugoniot jump conditions. In addition, modern shock capturing techniques can be easily incorporated taking advantage of the conservative formulation. This can dramatically improve the performance of the algorithm in the vicinity of sharp spatial gradients. In this paper, a Total Variation Dimishing (TVD) space-time approach [3] is used, combining suitable slope limiters with a one-step two-stage explicit TVD Runge-Kutta time integrator.

Furthermore, the current paper extends the applicability of the formulation to include the consideration of thermo-mechanical processes. This requires the inclusion of the first law of thermodynamics (or known as conservation of the total energy $E$ ) and the satisfaction of the second law through the entropy production. The fully coupled mixed $\boldsymbol{p}-\boldsymbol{F}-J-E$ system will then be supplemented with the simplest possible thermal-mechanical constitutive law for solids, namely Mie-Grüneisen equation of state [61]. For completeness and ease of understanding, the paper will present an eigenvalue analysis of the complete set of mixed system to ensure the satisfaction of the hyperbolicity, and thus material stability. A series of numerical examples will be examined to assess the robustness and capabilities of the mixed algorithm, yielding second order of convergence for velocitites and stresses.

The outline of the present paper is as follows. Section 2 introduces a set of generalised governing equations for large strain non-isothermal fast dynamics, supplemented with appropriate mechanical constitutive models and equations of state. This section ends with the study of the eigenstructure of the problem. Section 3 describes the methodology of edge-based vertex centred FVM. Linear reconstruction, slope limiter and Riemann solver are also presented. Section 4 introduces the TVD Runge-Kutta time integrator used for temporal discretisation and some necessary numerical corrections to preserve the angular momentum. Section 6 summarises the solution procedure of the proposed methodology. In section 7, an extensive set of numerical examples is presented to assess the performance of the proposed method and to draw some comparisons against previous results published by the authors $[3,5]$. Finally, section 8 summarises some concluding remarks and current directions of research. 


\section{Governing equations}

\subsection{Conservation law formulation}

Consider the motion of a continuum which occupies a volume $V$ in the reference configuration and a volume $v$ in the deformed configuration. The motion is defined through a deformation mapping $\boldsymbol{x}=\boldsymbol{x}(\boldsymbol{X}, t)$ which, in a mixed formulation, can be described through the following extended set of conservation laws $[5,58]$ :

$$
\begin{aligned}
\frac{\partial \boldsymbol{p}}{\partial t}-\mathrm{DIV} \boldsymbol{P} & =\rho_{0} \boldsymbol{b}, \\
\frac{\partial \boldsymbol{F}}{\partial t}-\mathrm{DIV}\left(\frac{1}{\rho_{0}} \boldsymbol{p} \otimes \boldsymbol{I}\right) & =\mathbf{0}, \\
\frac{\partial J}{\partial t}-\mathrm{DIV}\left(\frac{1}{\rho_{0}} \boldsymbol{H}_{\boldsymbol{F}}^{T} \boldsymbol{p}\right) & =0 \\
\frac{\partial E}{\partial t}-\mathrm{DIV}\left(\frac{1}{\rho_{0}} \boldsymbol{P}^{T} \boldsymbol{p}-\boldsymbol{Q}\right) & =s
\end{aligned}
$$

The material divergence DIV of a second order two-point tensor is defined by the contraction:

$$
(\operatorname{DIV} \boldsymbol{A})_{i}=\frac{\partial A_{i I}}{\partial X_{I}}
$$

where Einstein's summation convention is implied for repeated indices. Note that $\boldsymbol{p}=\rho_{0} \boldsymbol{v}$ is the linear momentum, $\rho_{0}$ is the density in the undeformed configuration, $\boldsymbol{v}=\frac{\partial \boldsymbol{x}(\boldsymbol{X}, t)}{\partial t}$ is the velocity field, $\boldsymbol{P}$ is the first Piola-Kirchhoff stress tensor, $\boldsymbol{b}$ is the body force vector per unit of mass, $\boldsymbol{F}=\frac{\partial \boldsymbol{x}(\boldsymbol{X}, t)}{\partial \boldsymbol{X}}$ is the deformation gradient (also known as fibre map), $J$ is the Jacobian of the deformation (also known as volume map), $E$ is the total energy per unit of undeformed volume, $\boldsymbol{Q}$ the heat flux and $s$ the heat source term. In addition, expression $\boldsymbol{H}_{\boldsymbol{F}}$ represents the cofactor of the deformation gradient $\boldsymbol{F}$ defined as $\boldsymbol{H}_{\boldsymbol{F}}:=J_{\boldsymbol{F}} \boldsymbol{F}^{-T}$ where $J_{\boldsymbol{F}}:=\operatorname{det} \boldsymbol{F}$. Additionally, the system of equations (1a) to (1d) need to satisfy Dirichlet boundary conditions (imposed linear momentum $\boldsymbol{p}$ ) on $\partial_{u} V$ and Neumann boundary conditions (imposed traction per unit undeformed area $\left.\boldsymbol{t}_{0}\right)$ on $\partial_{t} V$, where $\partial_{t} V \cup \partial_{u} V=\partial V$ and $\partial_{t} V \cap \partial_{u} V=\emptyset$. Different types of boundary conditions are represented in Figure 1.

By virtue of equations (1b) and (1c), $\boldsymbol{F}$ and $J$ are treated as independent variables not derived directly from the material gradient of a displacement 


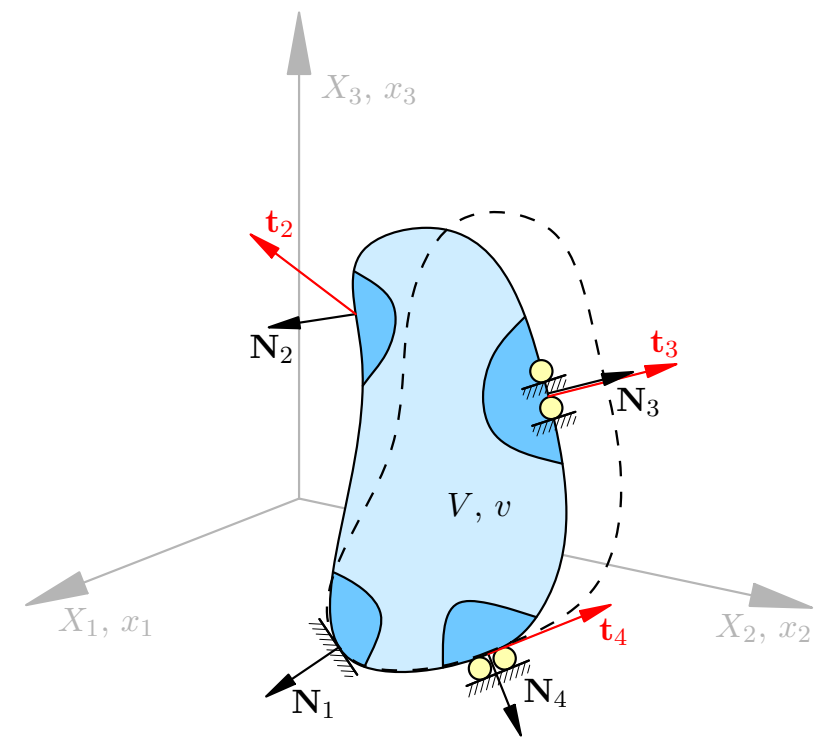

Figure 1: Different boundary conditions to be imposed. The continuous line represents the body at the reference (undeformed) configuration, while the discontinuous line the body at the spatial (deformed) configuration. Four different types of boundary conditions are considered: Fix (condition 1), Free (condition 2), Skew symmetric (condition 3) and Symmetric (condition $4)$. 
field, contrary to the classical displacement based formulation [33, 35, 3739]. Crucially, the evolution of the fibre map (1b) must be advanced in time satisfying a set of compatibility conditions (known as involutions [62]), namely

$$
\operatorname{CURL}(\boldsymbol{F})=\mathbf{0} \text {. }
$$

This ensures that $\boldsymbol{F}$ corresponds to the material gradient of a real mapping $[2,3,42]$. Notice that the material CURL of a second order two-point tensor is defined in the usual fashion by:

$$
(\mathrm{CURL} \boldsymbol{A})_{i I}=\mathcal{E}_{I J K} \frac{\partial A_{i K}}{\partial X_{J}}
$$

Equations (1a-1d) can be combined into a system of equations as

$$
\frac{\partial \mathcal{U}}{\partial t}+\operatorname{DIV} \mathcal{F}=\mathcal{S}
$$

where $\mathcal{U}$ is the vector of conservation variables, $\mathcal{F}$ denotes the flux matrix and $\mathcal{S}$ the source term, namely

$$
\mathcal{U}=\left[\begin{array}{c}
\boldsymbol{p} \\
\boldsymbol{F} \\
J \\
E
\end{array}\right], \mathcal{F}=-\left[\begin{array}{c}
\boldsymbol{P} \\
\frac{1}{\rho_{0}} \boldsymbol{p} \otimes \boldsymbol{I} \\
\frac{1}{\rho_{0}} \boldsymbol{H}_{\boldsymbol{F}}^{T} \boldsymbol{p} \\
\frac{1}{\rho_{0}} \boldsymbol{P}^{T} \boldsymbol{p}-\boldsymbol{Q}
\end{array}\right], \mathcal{S}=\left[\begin{array}{c}
\rho_{0} \boldsymbol{b} \\
\mathbf{0} \\
0 \\
s
\end{array}\right]
$$

The above conservation laws (5) accept weak solutions with discontinuities (or shocks) travelling at certain propagation speeds through the medium. Within the undeformed domain $V$, a material interface $\Gamma$ defined by the outward unit normal vector $\boldsymbol{N}$ can experience a jump in the conservation variables $\llbracket \mathcal{U} \rrbracket=\mathcal{U}^{+}-\mathcal{U}^{-}$travelling with speed $U$. The upper indices $\{-,+\}$ indicate both sides of the interface and $\boldsymbol{N}$ is defined pointing from - to + . Following a similar methodology to that in [3], a full set of Rankine-Hugoniot jump conditions for conservation variables of interest in this paper, this time 
with the inclusion of volume map conservation law, is as follows:

$$
\begin{aligned}
U \llbracket \boldsymbol{p} \rrbracket & =-\llbracket \boldsymbol{P} \rrbracket \boldsymbol{N} \\
U \llbracket \boldsymbol{F} \rrbracket & =-\frac{1}{\rho_{0}} \llbracket \boldsymbol{p} \rrbracket \otimes \boldsymbol{N} \\
U \llbracket J \rrbracket & =-\frac{1}{\rho_{0}} \llbracket \boldsymbol{p} \rrbracket \cdot \boldsymbol{H}_{\boldsymbol{F}} \boldsymbol{N} \\
U \llbracket E \rrbracket & =-\frac{1}{\rho_{0}} \llbracket \boldsymbol{P}^{T} \boldsymbol{p} \rrbracket \cdot \boldsymbol{N}+\llbracket \boldsymbol{Q} \rrbracket \cdot \boldsymbol{N},
\end{aligned}
$$

By virtue of (7c), it has been taken into account that $\boldsymbol{H}_{\boldsymbol{F}}$ has no jump in

the normal direction. This is due to the fact that $\mathbf{D I V} \boldsymbol{H}_{\boldsymbol{F}}=\mathbf{0}$ [58], and thus leads to $\llbracket \boldsymbol{H}_{\boldsymbol{F}} \rrbracket \boldsymbol{N}=\mathbf{0}$.

For the closure of above system (6), an appropriate constitutive law satisfying both the principle of objectivity and thermodynamic consistency (see Colemann-Noll procedure [63]) is supplemented, which will relate $\boldsymbol{P}$ with $\boldsymbol{F}$ and $J$.

\subsection{Isothermal processes}

In the case of isothermal process, equation (1d) is decoupled and does not need to be solved, except to possibly evaluate the numerical dissipation of the final algorithm.

\subsubsection{Compressible Mooney-Rivlin model}

The strain energy functional describing this constitutive model can be formulated as:

$W_{\mathrm{MR}}=\alpha \boldsymbol{F}: \boldsymbol{F}+\beta \boldsymbol{H}_{\boldsymbol{F}}: \boldsymbol{H}_{\boldsymbol{F}}+f(J) ; \quad f(J)=-4 \beta J-2 \alpha \ln J+\frac{\lambda}{2}(J-1)^{2}$,

where $\alpha$ and $\beta$ are positive material parameters, with $\alpha+\beta=\frac{\mu}{2}$, being $\lambda$ and $\mu$ the Lamé coefficients. The first Piola-Kirchhoff stress tensor can be simply derived by taking the derivative of the above functional $W_{\mathrm{MR}}(8)$ with respect to $\boldsymbol{F}$ and $J$ to give $[58,64]$

$$
\boldsymbol{P}=\frac{\partial W_{\mathrm{MR}}}{\partial \boldsymbol{F}}+f^{\prime}(J) \boldsymbol{H}_{\boldsymbol{F}} ; \quad f^{\prime}(J)=-4 \beta-\frac{2 \alpha}{J}+\lambda(J-1),
$$

where

$$
\frac{\partial W_{\mathrm{MR}}}{\partial \boldsymbol{F}}=2 \alpha \boldsymbol{F}+2 \beta\left[\frac{1}{J_{\boldsymbol{F}}}\left(\boldsymbol{H}_{\boldsymbol{F}}: \boldsymbol{H}_{\boldsymbol{F}}\right) \boldsymbol{H}_{\boldsymbol{F}}-J_{\boldsymbol{F}} \boldsymbol{H}_{\boldsymbol{F}} \boldsymbol{C}^{-1}\right] ; \quad \boldsymbol{C}=\boldsymbol{F}^{T} \boldsymbol{F} .
$$


In the particular case where $\beta=0$, the first Piola-Kirchhoff stress tensor of a compressible Neo-Hookean $(\mathrm{NH})$ model is simply recovered, given by [26]

$$
\boldsymbol{P}=\mu\left(\boldsymbol{F}-\frac{1}{J} \boldsymbol{H}_{\boldsymbol{F}}\right)+\lambda(J-1) \boldsymbol{H}_{\boldsymbol{F}} .
$$

The energy functional defined by (8) satisfies the so-called polyconvexity condition [59], ensuring hence rank one convexity, also known as the Legendre and Hadamard condition [63], which guarantees the existence of travelling waves with real wave speeds.

\subsubsection{Nearly incompressible Neo-Hookean model}

The energy functional describing this constitutive model is formulated (see for example $[26,31]$ ) with an additive decomposition into the summation

of a deviatoric strain energy $W_{\mathrm{NH}}^{\text {dev }}\left(J_{\boldsymbol{F}}^{-1 / 3} \boldsymbol{F}\right)$ and a volumetric strain energy $W_{\mathrm{NH}}^{\text {vol }}(J)$ defined by

$$
W_{\mathrm{NH}}^{d e v}=\frac{1}{2} \mu\left(J_{\boldsymbol{F}}^{-2 / 3}(\boldsymbol{F}: \boldsymbol{F})-3\right) ; \quad W_{\mathrm{NH}}^{v o l}=\frac{1}{2} \kappa(J-1)^{2},
$$

where $\kappa$ is the bulk modulus. The corresponding first Piola-Kirchhoff stress tensor can now be obtained as

$$
\boldsymbol{P}=\boldsymbol{P}^{d e v}+\boldsymbol{P}^{v o l}, \quad \boldsymbol{P}^{d e v}=\frac{\partial W_{\mathrm{NH}}^{d e v}}{\partial \boldsymbol{F}}, \quad \boldsymbol{P}^{v o l}=\frac{d W_{\mathrm{NH}}^{v o l}}{d J} \boldsymbol{H}_{\boldsymbol{F}}=p \boldsymbol{H}_{\boldsymbol{F}},
$$

where the deviatoric stress $\boldsymbol{P}^{d e v}$ and the pressure $p$ are

$$
\boldsymbol{P}^{d e v}=\mu J_{\boldsymbol{F}}^{-2 / 3}\left(\boldsymbol{F}-\frac{1}{3}(\boldsymbol{F}: \boldsymbol{F}) \boldsymbol{F}^{-T}\right), \quad p=\kappa(J-1) .
$$

It is possible to demonstrate that the above model satisfies the rank one convexity condition [5].

\subsection{Irreversible processes}

Two different types of irreversible processes are considered: strictly thermoelastic processes and isothermal elasto-plastic processes. The first one is implemented using the well-known Mie-Grüneisen equation of state, whereas the second a rate-independent von Mises plasticity model. 


\subsubsection{Mie-Grüneisen equation of state}

The total energy $E$ can be additively decomposed into a kinetic energy contribution $\frac{1}{2 \rho_{0}} \boldsymbol{p} \cdot \boldsymbol{p}$, a potential energy contribution $W_{\text {ext }}$ due to the presence of body forces $\rho_{0} \boldsymbol{b}$ and an internal energy contribution $e$ which includes strain and heat effects

$$
E=\frac{1}{2 \rho_{0}} \boldsymbol{p} \cdot \boldsymbol{p}+W_{e x t}+e ; \quad W_{e x t}=-\rho_{0} \boldsymbol{b} \cdot \boldsymbol{x} .
$$

Substitution of equations (1a) and (15) into (1d) enables the rate of internal energy $e$ to be obtained in non-conservative form as

$$
\frac{\partial e}{\partial t}=\boldsymbol{P}: \nabla_{0}\left(\frac{\boldsymbol{p}}{\rho_{0}}\right)-\mathrm{DIV} \boldsymbol{Q}+s
$$

where $\nabla_{0}$ is the material gradient operator described by $\left[\nabla_{0}\right]_{I} \equiv \frac{\partial}{\partial X_{I}}$.

In the case of strict thermoelasticity ${ }^{2}$, the internal energy $e$ can be defined in terms of the fibre map $\boldsymbol{F}$, the volume map $J$ and the specific entropy per unit of undeformed volume $\eta$, namely $e=e(\boldsymbol{F}, J, \eta)$. Moreover, the temperature $\theta$ can now be introduced as the state variable conjugate of the specific entropy $\eta$ as $\theta=\left.\frac{\partial e}{\partial \eta}\right|_{\boldsymbol{F}, J}{ }^{3}$. Considering the conservation laws for the fibre map $\boldsymbol{F}(1 \mathrm{~b})$ and the volume map $J(1 \mathrm{c})$, it is now possible to expand the time rate of the internal energy $e$ as,

$$
\frac{\partial e}{\partial t}=\left[\left.\frac{\partial e}{\partial \boldsymbol{F}}\right|_{J, \eta}+\left.\frac{\partial e}{\partial J}\right|_{\boldsymbol{F}, \eta} \boldsymbol{H}_{\boldsymbol{F}}\right]: \nabla_{0}\left(\frac{\boldsymbol{p}}{\rho_{0}}\right)+\theta \frac{\partial \eta}{\partial t} .
$$

Comparison of (16) and (17) leads to expressions for the Piola-Kirchhoff stress tensor and the evolution of the specific entropy in time, as follows,

$$
\boldsymbol{P}=\left.\frac{\partial e}{\partial \boldsymbol{F}}\right|_{J, \eta}+\left.\frac{\partial e}{\partial J}\right|_{\boldsymbol{F}, \eta} \boldsymbol{H}_{\boldsymbol{F}} ; \quad \frac{\partial \eta}{\partial t}=-\frac{1}{\theta} \operatorname{DIV} \boldsymbol{Q}+\frac{s}{\theta}
$$

\footnotetext{
${ }^{2}$ In the absence of other state variables such as plastic strain.

${ }^{3}$ For completeness, and in order to avoid ambiguity, the variables that remain fixed whilst carrying out the partial differentiation have been included, namely $\left.\frac{\partial e}{\partial \eta}\right|_{\boldsymbol{F}, J} \equiv$ $\frac{\partial e(\boldsymbol{F}, J, \eta)}{\partial \eta}$.
} 
By means of the Legendre transform, the Helmholtz's free energy per unit of undeformed volume $W=W(\boldsymbol{F}, J, \theta)$ can be introduced as

$$
W(\boldsymbol{F}, J, \theta)=e(\boldsymbol{F}, J, \eta)-\theta \eta
$$

leading to stress-strain relations at constant temperature

$$
\boldsymbol{P}=\left.\frac{\partial W}{\partial \boldsymbol{F}}\right|_{J, \theta}+\left.\frac{\partial W}{\partial J}\right|_{\boldsymbol{F}, \theta} \boldsymbol{H}_{\boldsymbol{F}} \quad \text { and } \quad \eta=-\left.\frac{\partial W}{\partial \theta}\right|_{\boldsymbol{F}, J} .
$$

It is possible to provide a relationship between the internal energy $e$ and the temperature $\theta$ through the specific heat coefficient at constant deformation, $C_{F}$, defined by the amount of energy to heat up a unit mass of material by a unit amount of temperature, as:

$$
\rho_{0} C_{F}=\left.\frac{\partial e}{\partial \theta}\right|_{\boldsymbol{F}, J}
$$

where, for simplicity, the specific heat coefficient $C_{F}$ is assumed to be temperature independent ${ }^{4}$. In this case, the internal energy $e$ can be obtained as:

$$
e(\boldsymbol{F}, J, \theta)=e_{0}(\boldsymbol{F}, J)+\rho_{0} C_{F} \Delta \theta ; \quad \Delta \theta=\theta-\theta_{0} ; \quad e_{0}(\boldsymbol{F}, J)=e\left(\boldsymbol{F}, J, \theta_{0}\right),
$$

where $\theta_{0}$ denotes a reference temperature in the undeformed configuration and $e_{0}(\boldsymbol{F}, J)$ represents the internal energy as a function of the deformation for a fixed reference temperature $\theta_{0}$. It is now possible to express the increment in temperature $\Delta \theta$ in terms of the internal energy of the system as

$\Delta \theta=\frac{1}{\rho_{0} C_{F}}\left[e(\boldsymbol{F}, J, \theta)-W_{0}(\boldsymbol{F}, J)-\theta_{0} \eta_{0}(\boldsymbol{F}, J)\right] ; \quad \eta_{0}(\boldsymbol{F}, J)=\eta\left(\boldsymbol{F}, J, \theta_{0}\right)$,

where use has been made of the Legendre transform (19) in the reference temperature state $\theta_{0}$, namely $W_{0}(\boldsymbol{F}, J)=e_{0}(\boldsymbol{F}, J)-\theta_{0} \eta_{0}(\boldsymbol{F}, J)$. In this case, $W_{0}(\boldsymbol{F}, J)$ and $\eta_{0}(\boldsymbol{F}, J)$ represent the Helmholtz's free energy and the

\footnotetext{
${ }^{4}$ Notice that in equation (21), the internal energy has been re-defined in terms of the variables $\boldsymbol{F}, J, \theta$. Hence, technically speaking, a different function $\tilde{e}$ should have been introduced, namely $e(\boldsymbol{F}, J, \eta) \equiv \tilde{e}(\boldsymbol{F}, J, \theta)$.
} 
entropy of the system as a function of the deformation for a fixed reference temperature $\theta_{0}$. Knowledge of expressions for $W_{0}(\boldsymbol{F}, J)$ and $\eta_{0}(\boldsymbol{F}, J)$ enable the computation of $\Delta \theta$, once the internal energy of the system is obtained via equations (1d) and (15).

Moreover, making use of the chain rule, equation (21) can be re-written as:

$$
\rho_{0} C_{F}=\left.\left.\frac{\partial e}{\partial \eta}\right|_{\boldsymbol{F}, J} \frac{\partial \eta}{\partial \theta}\right|_{\boldsymbol{F}, J}=\left.\theta \frac{\partial \eta}{\partial \theta}\right|_{\boldsymbol{F}, J},
$$

or

$$
\left.\frac{\partial \eta}{\partial \theta}\right|_{\boldsymbol{F}, J}=\frac{\rho_{0} C_{F}}{\theta}
$$

Above equation (25) can be integrated to give an expression for the entropy $\eta$ of the system, for a constant specific heat coefficient,

$$
\eta(\boldsymbol{F}, J, \theta)=\eta_{0}(\boldsymbol{F}, J)+\rho_{0} C_{F} \ln \left(\frac{\theta}{\theta_{0}}\right)
$$

Recalling equation (20b), further integration of above equation (26), yields

$W(\boldsymbol{F}, J, \theta)=W_{0}(\boldsymbol{F}, J)-\eta_{0}(\boldsymbol{F}, J) \Delta \theta+T(\theta) ; \quad T(\theta)=\rho_{0} C_{F}\left[\Delta \theta-\theta \ln \left(\frac{\theta}{\theta_{0}}\right)\right]$.

It is clear from above equation (27), that the Helmholtz's free energy function $W(\boldsymbol{F}, J, \theta)$ is decomposed additively into three terms: a purely mechanical term $W_{0}(\boldsymbol{F}, J)$, a purely thermal term $T(\theta)$ and a coupled term $\eta_{0}(\boldsymbol{F}, J)$. The term $W_{0}(\boldsymbol{F}, J)$ is usually defined via a standard hyperelastic constitutive model (see equations (8) or (12)) and thus, the complete closure of the system requires the definition of the entropy function $\eta_{0}(\boldsymbol{F}, J)$. In its simplest form, $\eta_{0}$ can be assumed to depend only on the Jacobian of the motion $J$, that is $\eta_{0}(\boldsymbol{F}, J)=\eta_{0}(J)$. Using (20a) and (27), the corresponding first Piola-Kirchoff stress tensor becomes

$$
\boldsymbol{P}(\boldsymbol{F}, J, \theta)=\boldsymbol{P}_{0}^{\operatorname{dev}}(\boldsymbol{F})+p(J, \theta) \boldsymbol{H}_{\boldsymbol{F}},
$$

with

$$
\boldsymbol{P}_{0}^{\operatorname{dev}}(\boldsymbol{F})=\frac{\partial W_{0}^{\operatorname{dev}}(\boldsymbol{F})}{\partial \boldsymbol{F}} ; \quad p(J, \theta)=p_{0}(J)-\Delta \theta \eta_{0}^{\prime}(J)
$$


where $p_{0}(J)$ is already defined in $(14 \mathrm{~b})$. A well known equation of state for solids that satisfies the condition $\eta_{0}=\eta_{0}(J)$ is the Mie-Grüneisen equation of state $[61,65]$, in which the Mie-Grüneisen coefficient $\Gamma$ is defined as

$$
\Gamma(J)=-\left.J \frac{\partial p}{\partial e}\right|_{J} ; \quad \Gamma(J)=\Gamma_{0} J^{q} .
$$

$\Gamma_{0}$ is a material parameter and the coefficient $q$ is usually taken to be 1 for solids and 0 for fluids. Application of the chain rule to equation (30) yields:

$$
\begin{aligned}
\Gamma(J) & =-\left.\left.J \frac{\partial p}{\partial \theta}\right|_{J} \frac{\partial \theta}{\partial e}\right|_{J} \\
& =-\left.J \frac{\partial p}{\partial \theta}\right|_{J} \frac{1}{\left.\frac{\partial e}{\partial \theta}\right|_{J}} \\
& =-\left.\frac{J}{\rho_{0} C_{F}} \frac{\partial p}{\partial \theta}\right|_{J} .
\end{aligned}
$$

Substitution of (29b) into (31) results in

$$
\eta_{0}^{\prime}(J)=\rho_{0} C_{F} \Gamma_{0} J^{(q-1)} .
$$

Using equation (32), the entropy at the reference temperature can now be integrated to give

$$
\eta_{0}(J)= \begin{cases}\rho_{0} C_{F} \Gamma_{0} \frac{\left(J^{q}-1\right)}{q} & \text { if } q>0 \\ \rho_{0} C_{F} \Gamma_{0} \ln J & \text { if } q=0,\end{cases}
$$

where the value of entropy at the reference temperature and undeformed configuration $\eta_{0}(J=1)$ has been taken as zero. Finally, substitution of equation (32) into (29b), the explicit expression for pressure becomes

$$
p(J, \theta)=p_{0}(J)-\rho_{0} C_{F} \Gamma_{0} J^{q-1} \Delta \theta,
$$

which evaluates the pressure in terms of the Jacobian of the deformation $J$ and the temperature increase $\Delta \theta$, the latter obtained via equations (23). Above equation (34) reduces in the case of an ideal gas to

$$
p(J, \theta)=-\rho_{0} C_{F} \Gamma_{0} J^{-1} \Delta \theta,
$$

based on two assumptions, namely $q=0$ and $W_{0}(\boldsymbol{F}, J)=0$. 
Another possible reference state is the locus of states behind a shock, named the Hugoniot locus. This locus is obtained by taking the conservation of mass, linear momentum and energy in Eulerian form and using the Rankine-Hugoniot jump conditions in the direction of the shock (see [50, 6567]) which yields

$$
\begin{aligned}
v_{H} & =c_{p}(1-J), \\
p_{H} & =-\rho_{0} c_{p} v_{H}, \\
e_{H} & =\frac{1}{2} \frac{\rho_{0}}{J} v_{H}^{2} .
\end{aligned}
$$

where the subscript $H$ refers to the state behind a shock and $c_{p}$ is the velocity of the shock. These equations are usually complemented with experimental data that relates the shock velocity $c_{p}$ to the particle velocity $v_{H}$ behind the shock in the form $c_{p}=c_{p}\left(v_{H}\right)$. For metals in the absence of phase transitions, it is proved that this relation is linear [65] and given by

$$
c_{p}=c_{p, 0}+s v_{H},
$$

where $c_{p, 0}$ is the volumetric wave speed of the material at rest and $s$ is a material depending parameter. Using $p_{H}$ and $e_{H}$ as reference presssure and energy and the Mie-Grüneisen formalism, the pressure behind a shock can be formulated as [50]

$$
p(e, J)=\frac{\rho_{0} c_{p, 0}^{2}(J-1)}{(1-s(1-J))^{2}}-\frac{\Gamma(J)}{J}\left[e-\frac{1}{2} \rho_{0} c_{p, 0}^{2}\left(\frac{(J-1)}{1-s(1-J)}\right)^{2}\right],
$$

The pressure associated to an adiabatic process (14), namely $p=\rho_{0} c_{p, 0}^{2}(J-$ 1 ), can be simply recovered when using $s=\Gamma_{0}=0$. With regard to gas dynamics, where $\kappa=q=0$, above equation (40) simplifies to

$$
p(J, e)=-\Gamma_{0} J^{-1} e .
$$

which can also be recovered from $(35)^{5}$.

\footnotetext{
${ }^{5}$ Notice the sign convention adopted in this paper that a negative value of pressure indicates compression.
} 


\subsubsection{Hyperelastic plastic model}

In order to model plastic behaviour, a rate-independent von Mises plasticity model with isotropic hardening, such as that presented in [26, 68], is used. The deformation gradient tensor $\boldsymbol{F}$ is multiplicatively decomposed into an elastic component $\boldsymbol{F}_{e}$ and a plastic component $\boldsymbol{F}_{p}$ as

$$
\boldsymbol{F}=\boldsymbol{F}_{e} \boldsymbol{F}_{p} ; \quad \boldsymbol{b}_{e}=\boldsymbol{F} \boldsymbol{C}_{p}^{-1} \boldsymbol{F}^{T} ; \quad \boldsymbol{C}_{p}=\boldsymbol{F}_{p}^{T} \boldsymbol{F}_{p}
$$

In addition, a strain energy functional in terms of the elastic principal stretches $\left(\lambda_{e, 1}, \lambda_{e, 2}, \lambda_{e, 3}\right)$ is defined as

$$
\psi\left(\lambda_{e, 1}, \lambda_{e, 2}, \lambda_{e, 3}\right)=\psi_{\text {dev }}\left(J^{-1 / 3} \lambda_{e, 1}, J^{-1 / 3} \lambda_{e, 2}, J^{-1 / 3} \lambda_{e, 3}\right)+\psi_{\text {vol }}(J)
$$

where

$$
\psi_{\text {dev }}=\mu\left[\left(\ln \lambda_{e, 1}\right)^{2}+\left(\ln \lambda_{e, 2}\right)^{2}+\left(\ln \lambda_{e, 3}\right)^{2}\right]-\frac{1}{3} \mu(\ln J)^{2}
$$

and

$$
\psi_{v o l}=\frac{1}{2} \kappa(\ln J)^{2} ; \quad \ln J=\ln \lambda_{e, 1}+\ln \lambda_{e, 2}+\ln \lambda_{e, 3}
$$

The algorithm to update the plastic strain $\boldsymbol{C}_{p}$ is summarised in Algorithm 
$2.1[26]$.

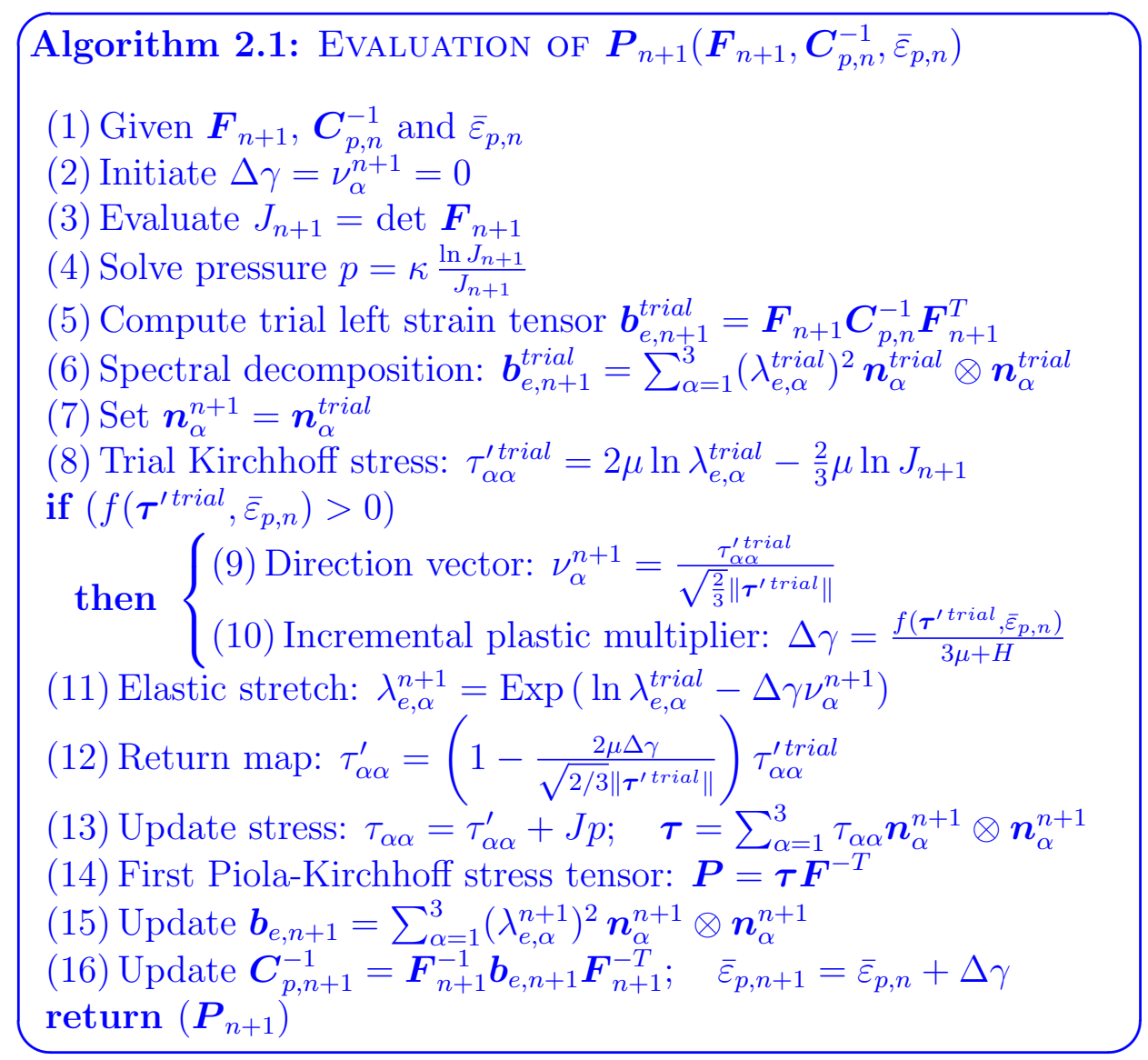

\subsection{Eigenvalue structure}

Satisfaction of rank one convexity [63] is equivalent to the existence of travelling waves with real wave speeds. Therefore, the study of the eigenvalue structure of the system of conservation laws becomes of paramount importance. The study of the eigenstructure of the hyperbolic system has been presented previously by other authors [2,3,5]. Extension of the analysis to include the conservation law of Jacobian and the consideration of thermo-mechanical processes is presented in this section.

The eigenvalues and eigenvectors of the system (5) can be evaluated con- 
sidering plane wave solutions of the form

$$
\mathcal{U}=\phi\left(\boldsymbol{X} \cdot \boldsymbol{N}-c_{\alpha} t\right) \overline{\mathcal{U}}_{\alpha}=\phi\left(\boldsymbol{X} \cdot \boldsymbol{N}-c_{\alpha} t\right)\left[\begin{array}{c}
\boldsymbol{p}_{\alpha} \\
\boldsymbol{F}_{\alpha} \\
J_{\alpha} \\
E_{\alpha}
\end{array}\right]
$$

where $c_{\alpha}$ are the wave speeds corresponding to the eigenvector $\overline{\mathcal{U}}_{\alpha}$. The resulting eigen-system is of the form

$$
c_{\alpha} \overline{\mathcal{U}}_{\alpha}=\mathcal{A}_{N} \overline{\mathcal{U}}_{\alpha} ; \quad \mathcal{A}_{N}=\mathcal{A}_{I} N_{I} ; \quad \mathcal{A}_{I}=\frac{\partial \mathcal{F}_{I}}{\partial \mathcal{U}}
$$

where $\mathcal{F}_{I}$ is the flux vector in the material direction $I$ and $\mathcal{A}_{N}$ is the flux Jacobian matrix of the system given by

$$
\boldsymbol{A}_{\boldsymbol{N}}=\left[\begin{array}{cccc}
-\frac{\partial(\boldsymbol{P} \boldsymbol{N})}{\partial \boldsymbol{p}} & -\frac{\partial(\boldsymbol{P} \boldsymbol{N})}{\partial \boldsymbol{F}} & -\frac{\partial(\boldsymbol{P N})}{\partial J} & -\frac{\partial(\boldsymbol{P} \boldsymbol{N})}{\partial E} \\
-\frac{\partial\left(\frac{1}{\rho_{0}} \boldsymbol{p} \otimes \boldsymbol{N}\right)}{\partial \boldsymbol{p}} & -\frac{\partial\left(\frac{1}{\rho_{0}} \boldsymbol{p} \otimes \boldsymbol{N}\right)}{\partial \boldsymbol{F}} & -\frac{\partial\left(\frac{1}{\rho_{0}} \boldsymbol{p} \otimes \boldsymbol{N}\right)}{\partial J} & -\frac{\partial\left(\frac{1}{\rho_{0}} \boldsymbol{p} \otimes \boldsymbol{N}\right)}{\partial E} \\
-\frac{\partial\left(\frac{1}{\rho_{0}} \boldsymbol{p} \cdot \boldsymbol{H}_{\boldsymbol{F}} \boldsymbol{N}\right)}{\partial \boldsymbol{p}} & -\frac{\partial\left(\frac{1}{\rho_{0}} \boldsymbol{p} \cdot \boldsymbol{H}_{\boldsymbol{F}} \boldsymbol{N}\right)}{\partial \boldsymbol{F}} & -\frac{\partial\left(\frac{1}{\rho_{0}} \boldsymbol{p} \cdot \boldsymbol{H}_{\boldsymbol{F}} \boldsymbol{N}\right)}{\partial J} & -\frac{\partial\left(\frac{1}{\rho_{0}} \boldsymbol{p} \cdot \boldsymbol{H}_{\boldsymbol{F}} \boldsymbol{N}\right)}{\partial E} \\
-\frac{\partial\left(\frac{1}{\rho_{0}} \boldsymbol{p} \cdot \boldsymbol{P} \boldsymbol{N}\right)}{\partial \boldsymbol{p}} & -\frac{\partial\left(\frac{1}{\rho_{0}} \boldsymbol{p} \cdot \boldsymbol{P} \boldsymbol{N}\right)}{\partial \boldsymbol{F}} & -\frac{\partial\left(\frac{1}{\rho_{0}} \boldsymbol{p} \cdot \boldsymbol{P} \boldsymbol{N}\right)}{\partial J} & -\frac{\partial\left(\frac{1}{\rho_{0}} \boldsymbol{p} \cdot \boldsymbol{P} \boldsymbol{N}\right)}{\partial E}
\end{array}\right] .
$$

A thorough study of the eigenstructure analysis of the three-field $\boldsymbol{p}$ - $\boldsymbol{F}$ $J$ system was carried out in detail in [5] for isothermal elasticity. In what follows, the eigen-analysis will be extended to the consideration of thermoelasticity together with a Mie-Grüneisen equation of state. Upon substitution of equations (29) and (33) into (48), it yields

$$
\mathcal{A}_{\boldsymbol{N}}=\left[\begin{array}{cccc}
{\left[\mathcal{A}_{\boldsymbol{N}}\right]_{\boldsymbol{p}, \boldsymbol{p}}} & {\left[\boldsymbol{\mathcal { A }}_{\boldsymbol{N}}\right]_{\boldsymbol{p}, \boldsymbol{F}}} & {\left[\boldsymbol{\mathcal { A }}_{\boldsymbol{N}}\right]_{\boldsymbol{p}, J}} & {\left[\boldsymbol{\mathcal { A }}_{\boldsymbol{N}}\right]_{\boldsymbol{p}, E}} \\
-\frac{1}{\rho_{0}} \boldsymbol{I}_{\boldsymbol{N}} & \mathbf{0}_{3 \times 3 \times 3 \times 3} & \mathbf{0}_{3 \times 3} & \mathbf{0}_{3 \times 3} \\
-\frac{1}{\rho_{0}} \boldsymbol{H}_{\boldsymbol{F}} \boldsymbol{N} & -\left(\frac{1}{\rho_{0}} \boldsymbol{p} \otimes \boldsymbol{N}: \frac{\partial \boldsymbol{H}_{\boldsymbol{F}}}{\partial \boldsymbol{F}}\right) & 0 & 0 \\
-\frac{1}{\rho_{0}} \boldsymbol{P} \boldsymbol{N}+\frac{1}{\rho_{0}} \boldsymbol{p}^{T}\left[\mathcal{A}_{\boldsymbol{N}}\right]_{\boldsymbol{p}, \boldsymbol{p}} & \frac{1}{\rho_{0}} \boldsymbol{p}^{T}\left[\mathcal{A}_{\boldsymbol{N}}\right]_{\boldsymbol{p}, \boldsymbol{F}} & \frac{1}{\rho_{0}} \boldsymbol{p} \cdot\left[\boldsymbol{\mathcal { A }}_{\boldsymbol{N}}\right]_{\boldsymbol{p}, J} & \frac{1}{\rho_{0}} \boldsymbol{p} \cdot\left[\mathcal{A}_{\boldsymbol{N}}\right]_{\boldsymbol{p}, E}
\end{array}\right]
$$


where

$$
\begin{aligned}
& {\left[\mathcal{A}_{\boldsymbol{N}}\right]_{\boldsymbol{p}, \boldsymbol{p}}=\eta_{0}^{\prime}(J)\left(\boldsymbol{H}_{\boldsymbol{F}} \boldsymbol{N}\right) \otimes\left(\frac{\partial \Delta \theta}{\partial \boldsymbol{p}}\right)} \\
& {\left[\mathcal{A}_{\boldsymbol{N}}\right]_{\boldsymbol{p}, \boldsymbol{F}}=-\mathcal{C}_{\boldsymbol{N}}+\eta_{0}^{\prime}(J)\left(\boldsymbol{H}_{\boldsymbol{F}} \boldsymbol{N}\right) \otimes \frac{\partial \Delta \theta}{\partial \boldsymbol{F}}+\Delta \theta \eta_{0}^{\prime}(J) \frac{\partial\left(\boldsymbol{H}_{\boldsymbol{F}} \boldsymbol{N}\right)}{\partial \boldsymbol{F}}} \\
& {\left[\mathcal{A}_{\boldsymbol{N}}\right]_{\boldsymbol{p}, J}=-p_{0}^{\prime}(J)\left(\boldsymbol{H}_{\boldsymbol{F}} \boldsymbol{N}\right)+\eta_{0}^{\prime \prime}(J) \Delta \theta\left(\boldsymbol{H}_{\boldsymbol{F}} \boldsymbol{N}\right)+\eta_{0}^{\prime}(J) \frac{\partial \Delta \theta}{\partial J}\left(\boldsymbol{H}_{\boldsymbol{F}} \boldsymbol{N}\right)} \\
& {\left[\mathcal{A}_{\boldsymbol{N}}\right]_{\boldsymbol{p}, E}=\eta_{0}^{\prime}(J) \frac{\partial \Delta \theta}{\partial E}\left(\boldsymbol{H}_{\boldsymbol{F}} \boldsymbol{N}\right)} \\
& {\left[\mathcal{C}_{\boldsymbol{N}}\right]_{i j J}=[\mathcal{C}]_{i I j J} N_{I} ; \quad[\mathcal{C}]_{i I j J}=\frac{\partial[\boldsymbol{P}]_{i I}}{\partial[\boldsymbol{F}]_{j J}} ; \quad\left[\boldsymbol{I}_{\boldsymbol{N}}\right]_{i J k}=\delta_{i k} N_{J} .}
\end{aligned}
$$

Substituion of the expression for $\mathcal{A}_{\boldsymbol{N}}$ derived above into (47a), and particularisation for each of the conservation variables, gives

$$
\begin{aligned}
& c_{\alpha} \boldsymbol{p}_{\alpha}= {\left[\eta_{0}^{\prime}(J)\left(\boldsymbol{H}_{\boldsymbol{F}} \boldsymbol{N}\right) \otimes\left(\frac{\partial \Delta \theta}{\partial \boldsymbol{p}}\right)\right] \boldsymbol{p}_{\alpha} } \\
&+ {\left[-\mathcal{C}_{\boldsymbol{N}}+\eta_{0}^{\prime}(J)\left(\boldsymbol{H}_{\boldsymbol{F}} \boldsymbol{N}\right) \otimes \frac{\partial \Delta \theta}{\partial \boldsymbol{F}}+\Delta \theta \eta_{0}^{\prime}(J) \frac{\partial\left(\boldsymbol{H}_{\boldsymbol{F}} \boldsymbol{N}\right)}{\partial \boldsymbol{F}}\right]: \boldsymbol{F}_{\alpha} } \\
&+ {\left[-p_{0}^{\prime}(J)\left(\boldsymbol{H}_{\boldsymbol{F}} \boldsymbol{N}\right)+\eta_{0}^{\prime \prime}(J) \Delta \theta\left(\boldsymbol{H}_{\boldsymbol{F}} \boldsymbol{N}\right)+\eta_{0}^{\prime}(J) \frac{\partial \Delta \theta}{\partial J}\left(\boldsymbol{H}_{\boldsymbol{F}} \boldsymbol{N}\right)\right] J_{\alpha} } \\
&+ {\left[\eta_{0}^{\prime}(J) \frac{\partial \Delta \theta}{\partial E}\left(\boldsymbol{H}_{\boldsymbol{F}} \boldsymbol{N}\right)\right] E_{\alpha} } \\
& c_{\alpha} \boldsymbol{F}_{\alpha}=-\frac{1}{\rho_{0}} \boldsymbol{p}_{\alpha} \otimes \boldsymbol{N}, \\
& c_{\alpha} J_{\alpha}=-\frac{1}{\rho_{0}}(\boldsymbol{H} \boldsymbol{F}) \cdot \boldsymbol{p}_{\alpha}-\frac{1}{\rho_{0}}\left[\boldsymbol{p} \otimes \boldsymbol{N}: \frac{\partial \boldsymbol{H}_{\boldsymbol{F}}}{\partial \boldsymbol{F}}: \boldsymbol{F}_{\alpha}\right] \\
& c_{\alpha} E_{\alpha}=-\frac{1}{\rho_{0}} \boldsymbol{P} \boldsymbol{N} \cdot \boldsymbol{p}_{\alpha}+\frac{c_{\alpha}}{\rho_{0}} \boldsymbol{p} \cdot \boldsymbol{p}_{\alpha} .
\end{aligned}
$$

Using equation (51b) and some algebra, it is possible to show that the squared bracket term on the right hand side of (51c) simply vanishes. Substituting (51b), (51c) and (51d) into (51a), together with the aid of (23), renders

$\rho_{0} c_{\alpha}^{2} \boldsymbol{p}_{\alpha}=\left[\mathcal{C}_{\boldsymbol{N} N}+p_{0}^{\prime}(J) \boldsymbol{m} \otimes \boldsymbol{m}\right] \boldsymbol{p}_{\alpha}+\left[\left(\frac{1}{\rho_{0} C_{F}} \theta\left(\eta_{0}^{\prime}(J)\right)^{2}-\eta_{0}^{\prime \prime}(J) \Delta \theta\right) \boldsymbol{m} \otimes \boldsymbol{m}\right] \boldsymbol{p}_{\alpha}$, 
where $\boldsymbol{m}=\boldsymbol{H}_{\boldsymbol{F}} \boldsymbol{N}$. Instead of deriving a close form solution for the expression above (52), it is just sufficient to obtain bounds of the wave speeds by assuming $\boldsymbol{N}$ is a principal direction. In this particular case, $\boldsymbol{m}=\frac{J_{\boldsymbol{F}}}{\lambda} \boldsymbol{n}$ where $\lambda$ represents stretch in the spatial direction $\boldsymbol{n}$. This would yield

$\rho_{0} c_{\alpha}^{2} \boldsymbol{p}_{\alpha}=\left[\mathcal{C}_{\boldsymbol{N} \boldsymbol{N}}+\left(p_{0}^{\prime}(J)+\frac{1}{\rho_{0} C_{F}} \theta\left(\eta_{0}^{\prime}(J)\right)^{2}-\eta_{0}^{\prime \prime}(J) \Delta \theta\right)\left(\frac{J_{F}}{\lambda}\right)^{2} \boldsymbol{n} \otimes \boldsymbol{n}\right] \boldsymbol{p}_{\alpha}$.

The above system contains three pairs of non-zero eigenvalues that correspond to the volumetric and shear waves as

$$
c_{1,2}= \pm c_{p}, \quad c_{p}=\sqrt{c_{0, p}^{2}-\frac{\eta_{0}^{\prime \prime}(J) \Delta \theta J_{F}^{2}}{\rho_{0} \lambda^{2}}+\frac{\left(\eta_{0}^{\prime}(J)\right)^{2} \theta J_{F}^{2}}{\rho_{0}^{2} C_{F} \lambda^{2}}}, \quad c_{3,4}=c_{5,6}= \pm c_{s, 0}
$$

with eigenvectors

$$
\begin{gathered}
\mathcal{U}_{1,2}=\left[\begin{array}{c}
\boldsymbol{n} \\
\pm \frac{1}{\rho_{0} c_{p}} \boldsymbol{n} \otimes \boldsymbol{N} \\
\pm \frac{1}{\rho_{0} c_{p}} \frac{J_{F}}{\lambda} \\
\pm \frac{1}{\rho_{0} c_{\alpha}} \boldsymbol{P} \cdot \boldsymbol{n}+\frac{1}{\rho_{0}} \boldsymbol{p} \cdot \boldsymbol{n}
\end{array}\right], \quad \boldsymbol{U}_{3,4}=\left[\begin{array}{c}
\boldsymbol{t}_{1} \\
\pm \frac{1}{\rho_{0} c_{s}} \boldsymbol{t}_{1} \otimes \boldsymbol{N} \\
0 \\
\pm \frac{1}{\rho_{0} c_{s}} \boldsymbol{P} \boldsymbol{N} \cdot \boldsymbol{t}_{1}+\frac{1}{\rho_{0}} \boldsymbol{p} \cdot \boldsymbol{t}_{1}
\end{array}\right] \\
\boldsymbol{U}_{5,6}=\left[\begin{array}{c} 
\pm \frac{1}{\rho_{0} c_{s}} \boldsymbol{t}_{2} \otimes \boldsymbol{N} \\
0 \\
\pm \frac{1}{\rho_{0} c_{s}} \boldsymbol{P} \boldsymbol{N} \cdot \boldsymbol{t}_{2}+\frac{1}{\rho_{0}} \boldsymbol{p} \cdot \boldsymbol{t}_{2}
\end{array}\right] .
\end{gathered}
$$

Note that $\boldsymbol{t}_{1}, \boldsymbol{t}_{2}$ are two arbitrary tangential vectors orthogonal to $\boldsymbol{n}$ while $c_{0, p}$ and $c_{0, s}$ are the wave speeds of (49) at reference temperature (see [5], equation (55), pg. 671), given by

$$
\rho_{0} c_{0, p}^{2} \boldsymbol{n}=\left[\mathcal{C}_{\boldsymbol{N} \boldsymbol{N}}+p_{0}^{\prime}(J)\left(\frac{J_{F}}{\lambda}\right)^{2} \boldsymbol{I}\right] \boldsymbol{n} ; \quad \rho_{0} c_{0, s}^{2} \boldsymbol{t}_{1,2}=\mathcal{C}_{\boldsymbol{N} \boldsymbol{N}} \boldsymbol{t}_{1,2} .
$$

Insofar as the Mie-Grüneisen equation is considered, the volumetric wave speed becomes:

$$
c_{p}=\sqrt{c_{0, p}^{2}+\frac{C_{F} \Gamma_{0} J_{F}^{2} J^{q-2}}{\lambda^{2}}\left[(1-q) \Delta \theta+\Gamma_{0} J^{q} \theta\right]} .
$$


To ensure the hyperbolicity of the system, the following inequality needs to be satisfied

$$
(1-q) \Delta \theta+\Gamma_{0} J^{q} \theta>0 .
$$

This is easy to prove noticing that all coefficients are positive since $0 \leq q \leq 1$.

\section{Finite volume spatial discretisation}

\subsection{Dual mesh and area vectors}

This section will present the spatial discretisation of a first order hyperbolic system of equations (5) using an edge-based upwind vertex centred Finite Volume Method (FVM). Following references [1, 69, 70], the upwind FVM requires the definition of a dual mesh, which will be constructed using the median dual approach [71].

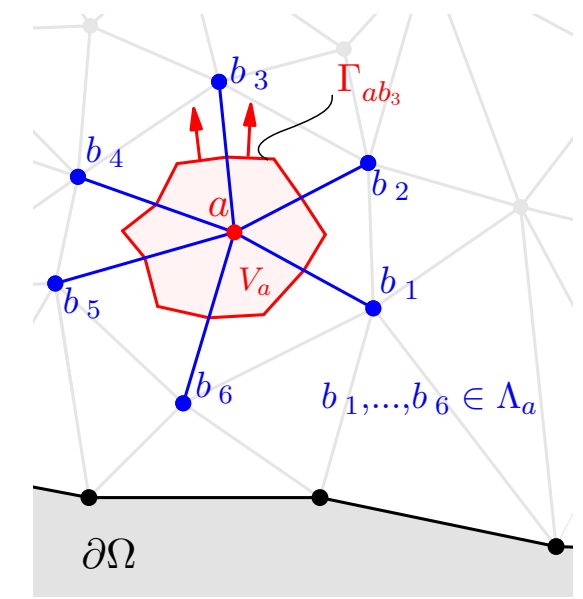

(a)

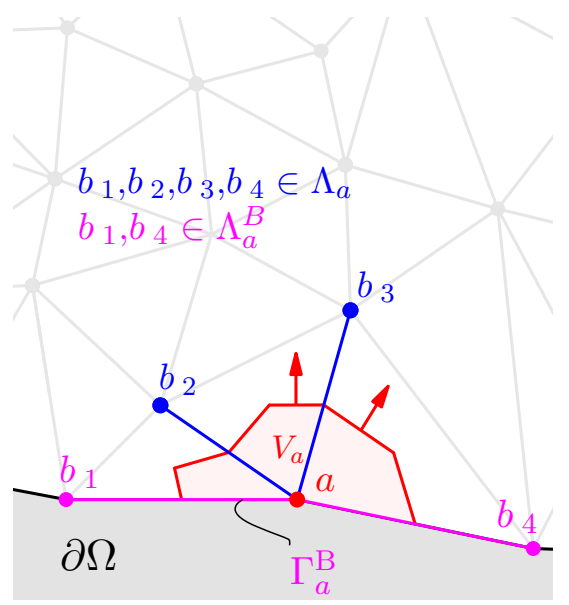

(b)

Figure 2: Control volume for (a) an interior node and (b) a boundary node using the median dual approach in a two-dimensional triangular mesh. The red shaded area is the control volume associated to node $a$. The blue lines are the edges connecting node $a$ to its neighbouring nodes $b_{i}$, that is, the set $\Lambda_{a}$. The magenta lines are the boundary edges connecting node $a$ to its neighbouring nodes $b_{i}$, that is, the subset $\Lambda_{a}^{B}$.

The dual mesh is constructed by connecting edge midpoints with element centroids in two dimensions (see Figure 2a,b) and edge midpoints with face 
centroids and element centroids in three dimensions. For a given node $a$, the set of nodes connected to it through an edge is denoted by $\Lambda_{a}$ (see Figure 2a) and the subset of nodes connected to $a$ through a boundary edge is written as $\Lambda_{a}^{B}$ (see Figure $2 \mathrm{~b}$ ). For a given edge connecting nodes $a$ and $b$, an area vector is then defined as

$$
\boldsymbol{C}_{a b}=\sum_{k \in \Gamma_{a b}} A_{k} \boldsymbol{N}_{k}
$$

where $\Gamma_{a b}$ is the set of facets belonging to edge $a b, A_{k}$ is the area of a given facet $k$ and $\boldsymbol{N}_{k}$ its outward unit normal. Due to the definition of the dual mesh, the area vectors satisfy $\boldsymbol{C}_{a b}=-\boldsymbol{C}_{b a}$. These area vectors enable a substantial reduction in the computational cost when computing the boundary integral used in the Green Gauss divergence theorem, since they save an additional loop on facets per edge $a b$. In the case of a boundary edge, the contribution of the boundary facets to which it belongs has to be taken into account. This set of faces will be defined as $\Gamma_{a}^{B}$ (see Figure $2 b$ ).

By using this approach, the discretisation of the fluxes using centred differences is equivalent to a classical Bubnov Galerkin spatial discretisation with linear elements [72-74]. This type of dual mesh also allows for the computation of the gradients by means of the Green-Gauss approach [75].

\subsection{Integration of the fluxes}

Consider a hyperbolic system of conservation laws (5) where $\mathcal{U}$ is the vector of conserved variables $(6 a), \mathcal{F}$ represents the flux matrix $(6 \mathrm{~b})$ and the source term $\mathcal{S}$ has been neglected for simplicity. This expression is integrated within a given control volume $a$ followed by the divergence theorem to give,

$$
V_{a} \frac{d \mathcal{U}_{a}}{d t}=-\int_{\partial V_{a}} \mathcal{F} \boldsymbol{N} d A
$$

where $\mathcal{U}_{a}$ is the average value of the variable within the control volume $V_{a}$ and $\boldsymbol{N}$ is the outward unit normal vector associated to the boundary $\partial V_{a}$. Equation (59) can be spatially approximated by means of an upwind $\mathrm{FVM}^{6}$ :

$$
V_{a} \frac{d \mathcal{U}_{a}}{d t}=-\sum_{b \in \Lambda_{a}} \sum_{k \in \Gamma_{a b}} \mathcal{F}_{\boldsymbol{N}_{k}}^{C} A_{k}-\sum_{\gamma \in \Gamma_{a}^{B}} \mathcal{F}_{a}^{\gamma} \boldsymbol{N}_{\gamma} \frac{A_{\gamma}}{3}
$$

\footnotetext{
${ }^{6}$ Note that the boundary contributions associated to node $a$ are discretised using a mesh of linear 4-noded tetrahedral elements whose partial nodal area is one-third of the surface area $A_{\gamma}[60]$.
} 
where $\mathcal{F}_{\boldsymbol{N}_{k}}^{C}=\mathcal{F}_{\boldsymbol{N}}^{C}\left(\mathcal{U}_{a}, \mathcal{U}_{b}, \boldsymbol{N}_{k}\right)$ is the normal contact flux at facet $k$. This contact flux is obtained as the solution of the Riemann problem established by the contact discontinuity at each of the facets. In the specific case of a physical interface (i.e. several materials present in a unique solid), this would have to be treated as a new numerical interface. It would result into duplication of nodes at each side, creation of their respective control volumes and the enforcement of continuity via a Riemann solver.

A wide variety of approximate Riemann solvers $[60,76]$ exist in the literature, being the ones of Roe [77] and Osher [78] the most popular. For (small) numerical jumps, it is also possible to derive a linearised acoustic Riemann solver by means of the Rankine-Hugoniot jump conditions, where the shock speed $U$ in (7) is simply replaced by the speed of sound in a material $[3,79]$. This will be the approach followed in this paper.

By virtue of (60), the spatial discretisation requires the computation of a contact flux per each of the facets $k$ associated to a given edge $a b$. This is not necessary when using linear elements [80] and the solution may be assumed to be constant for all the facets grouped at a particular edge. With the aid of a normalised area vector (58), defined as $\boldsymbol{N}_{a b}=\frac{\boldsymbol{C}_{a b}}{\left\|\boldsymbol{C}_{a b}\right\|}$, a unique normal contact flux at the midpoint of each edge $\mathcal{F}_{\boldsymbol{N}_{a b}}^{C}$ can be obtained. This would simplify equation (60) to

$$
V_{a} \frac{d \mathcal{U}_{a}}{d t}=-\sum_{b \in \Lambda_{a}} \mathcal{F}_{\boldsymbol{N}_{a b}}^{C}\left\|\boldsymbol{C}_{a b}\right\|-\sum_{\gamma \in \Gamma_{a}^{B}} \mathcal{F}_{a}^{\gamma} \boldsymbol{N}_{\gamma} \frac{A_{\gamma}}{3}
$$

where $\mathcal{F}_{\boldsymbol{N}_{a b}}^{C}=\mathcal{F}_{\boldsymbol{N}}^{C}\left(\mathcal{F}\left(\mathcal{U}_{a}\right), \mathcal{F}\left(\mathcal{U}_{b}\right), \boldsymbol{N}_{a b}\right)$ is the normal contact flux associated to edge $a b$. Comparing equation (60) and equation (61), the former requires to store the complex facet stencil grouped at each edge, whereas the latter requires to store the nodal variables and the edge structure. Hence, expression (61) can be solved using a unique loop on edges, resulting into a computationally more efficient discretisation. More importantly, the use of this simplification does not compromise the overall accuracy of the scheme (see for example [81], where a unique Roe Riemann Solver is used per area vector).

It is worth noting that equation (61) would only lead to a first order solution in space [76] since $\mathcal{U}_{a}$ and $\mathcal{U}_{b}$ are modelled following a piecewise constant representation. To improve this, a suitable reconstruction procedure over each control volume will be discussed in the next section. 


\subsection{Piecewise linear reconstruction}

To obtain second order accuracy in space, the numerical contact fluxes, $\mathcal{F}_{\boldsymbol{N}_{a b}}^{C}$ need to be evaluated based upon the piecewise linear reconstructed variables of interest in this paper. Particularly, the numerical contact flux at each edge depends on the actual fluxes of the conservation variables at its two ends. This reconstruction can be achieved using two different strategies. The first standard approach is to reconstruct the conservation variables $\left(\mathcal{U}_{a b}^{-}\right.$and $\left.\mathcal{U}_{a b}^{+}\right)$, followed by the computation of the corresponding fluxes at each contact regions $\left(\mathcal{F}\left(\mathcal{U}_{a b}^{-}\right)\right.$and $\left.\mathcal{F}\left(\mathcal{U}_{a b}^{+}\right)\right)$. The second strategy is to first compute the fluxes at each control volume $\left(\mathcal{F}\left(\mathcal{U}_{a}\right)\right.$ and $\left.\mathcal{F}\left(\mathcal{U}_{b}\right)\right)$ and then reconstruct the computed fluxes to the contact surface $\left(\mathcal{F}_{a b}^{-}\right.$and $\left.\mathcal{F}_{a b}^{+}\right)$. The latter is of particular interest since it will require less evaluations of the variable fluxes, and complex constitutive models are typically used in solid mechanics, resulting in a faster algorithm.

The numerical flux evaluation expressed in the first term on the righthand side of (61) can now be written in terms of the reconstructed fluxes on either side of the edge:

$$
\mathcal{F}_{\boldsymbol{N}_{a b}}^{C}=\mathcal{F}_{\boldsymbol{N}}^{C}\left(\mathcal{F}_{a b}^{-}, \mathcal{F}_{a b}^{+}, \boldsymbol{N}_{a b}\right)
$$

where

$$
\mathcal{F}_{a b}^{-}=\mathcal{F}\left(\mathcal{U}_{a}\right)+\frac{1}{2} \phi_{a} \boldsymbol{G}_{a} \Delta \boldsymbol{X} ; \quad \mathcal{F}_{a b}^{+}=\mathcal{F}\left(\mathcal{U}_{b}\right)-\frac{1}{2} \phi_{b} \boldsymbol{G}_{b} \Delta \boldsymbol{X} .
$$

Here, $\Delta \boldsymbol{X}=\boldsymbol{X}_{b}-\boldsymbol{X}_{a}, \phi_{\{a, b\}}$ are the slope limiters [75] and the gradient $\boldsymbol{G}$ is approximated through the use of Green-Gauss approach [75] defined as:

$$
V_{a} \boldsymbol{G}_{a}=\sum_{b \in \Lambda_{a}} \frac{\mathcal{F}\left(\mathcal{U}_{a}\right)+\mathcal{F}\left(\mathcal{U}_{b}\right)}{2} \otimes \boldsymbol{C}_{a b}+\sum_{\gamma \in \Gamma_{a}^{B}}\left(\mathcal{F}\left(\mathcal{U}_{a}\right) \otimes \boldsymbol{N}_{\gamma}\right) \frac{A_{\gamma}}{3}
$$

The remaining unknown to be determined in (62) is the definition of numerical contact flux at mid-edge $\mathcal{F}_{\boldsymbol{N}_{a b}}^{C}$, which will be discussed in the following section.

Observe that the solutions exhibit undershoots and overshoots in the vicinity of sharp spatial gradients if $\phi=1$. The inclusion of the slope limiter is to prevent the creation of new local extrema at flux integration points, fulfilling the principle of local maximum [82].

Following is the Barth and Jespersen limiter [83] used in this paper: 
1. Find the smallest and largest averaged values among adjacent control volumes $V_{b}$ and the current control volume $V_{a}$ :

$$
\mathcal{U}_{a}^{\min }=\min _{b \in \Lambda_{a}}\left(\mathcal{U}_{a}, \mathcal{U}_{b}\right) \quad \text { and } \quad \mathcal{U}_{a}^{\max }=\max _{b \in \Lambda_{a}}\left(\mathcal{U}_{a}, \mathcal{U}_{b}\right)
$$

2. Compute an unlimited reconstructed value at each flux integration point, namely

$$
\mathcal{U}_{a b}^{-}=\mathcal{U}_{a}+\frac{1}{2} \boldsymbol{G}_{a} \Delta \boldsymbol{X}
$$

3. Obtain a maximum allowable value of $\phi_{a b}$ for each edge $a b$.

$$
\phi_{a b}=\left\{\begin{array}{cl}
\min \left(1, \frac{\mathcal{U}_{a}^{\max }-\mathcal{U}_{a}}{\mathcal{U}_{a b}^{-}-\mathcal{U}_{a}}\right), & \text { if } \quad \mathcal{U}_{a b}^{-}-\mathcal{U}_{a}>0 \\
\min \left(1, \frac{\mathcal{U}_{a}^{\text {min }}-\mathcal{U}_{a}}{\mathcal{U}_{a b}^{-}-\mathcal{U}_{a}}\right), & \text { if } \quad \mathcal{U}_{a b}^{-}-\mathcal{U}_{a}<0 \\
1, & \text { if } \quad \mathcal{U}_{a b}^{-}-\mathcal{U}_{a}=0
\end{array}\right.
$$

4. Select $\phi_{a}=\min _{b \in \Lambda_{a}}\left(\phi_{a b}\right)$.

\subsection{Numerical contact flux: linearised Riemann solver}

The upwind finite volume spatial discretisation (61) involving the definition of the numerical contact flux can be particularised into a set of conservation variables of interest in this paper, as follows:

$$
\begin{aligned}
V_{a} \frac{d \boldsymbol{p}_{a}}{d t} & =\sum_{b \in \Lambda_{a}} \boldsymbol{t}^{C}\left\|\boldsymbol{C}_{a b}\right\|+\sum_{\gamma \in \Gamma_{a}^{B}} \boldsymbol{t}_{a}^{\gamma} \frac{A_{\gamma}}{3} ; \\
V_{a} \frac{d \boldsymbol{F}_{a}}{d t} & =\sum_{b \in \Lambda_{a}} \frac{1}{\rho_{0}} \boldsymbol{p}^{C} \otimes \boldsymbol{C}_{a b}+\sum_{\gamma \in \Gamma_{a}^{B}} \frac{1}{\rho_{0}}\left(\boldsymbol{p}_{a}^{\gamma} \otimes \boldsymbol{N}_{\gamma}\right) \frac{A_{\gamma}}{3} ; \\
V_{a} \frac{d J_{a}}{d t} & =\sum_{b \in \Lambda_{a}} \frac{1}{\rho_{0}}\left(\boldsymbol{H}_{\boldsymbol{F}_{a}}^{T} \boldsymbol{p}^{C}\right) \cdot \boldsymbol{C}_{a b}+\sum_{\gamma \in \Gamma_{a}^{B}} \frac{1}{\rho_{0}}\left(\boldsymbol{H}_{\boldsymbol{F}_{a}}^{T} \boldsymbol{p}_{a}^{\gamma}\right) \cdot \boldsymbol{N}_{\gamma} \frac{A_{\gamma}}{3} ; \\
V_{a} \frac{d E_{a}}{d t} & =\sum_{b \in \Lambda_{a}} \frac{1}{\rho_{0}}\left(\boldsymbol{t}^{C} \cdot \boldsymbol{p}^{C}\right)\left\|\boldsymbol{C}_{a b}\right\|+\sum_{\gamma \in \Gamma_{a}^{B}} \frac{1}{\rho_{0}}\left(\boldsymbol{t}_{a}^{\gamma} \cdot \boldsymbol{p}_{a}^{\gamma}\right) \frac{A_{\gamma}}{3},
\end{aligned}
$$

where $\boldsymbol{t}^{C}$ and $\boldsymbol{p}^{C}$ are the contact traction and contact linear momentum, yet to be defined. In applying the contact linear momentum $\boldsymbol{p}^{C}$ in $(65 \mathrm{~b})$, the deformation gradient $\boldsymbol{F}$ will no longer be a discrete gradient of a continuous 


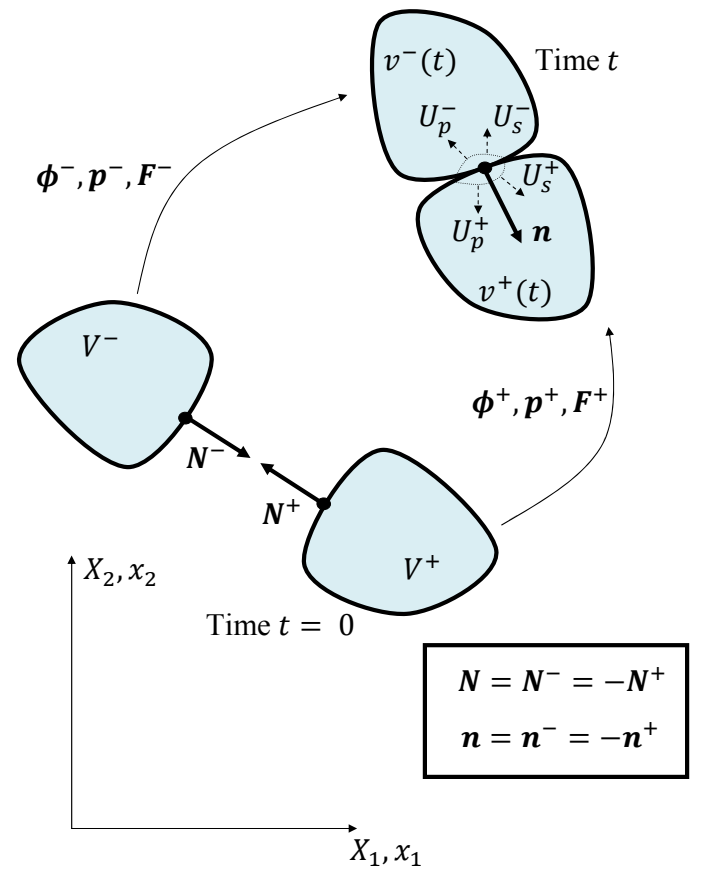

Figure 3: Contact conditions

velocity field. This implies that the involutions described by equation (3) are no longer enforced. For this reason, the contact linear momentum should only be applied to the third and fourth equations above for $J$ and $E$. The central difference approximation (equivalent to a linear Galerkin discretisation [7274]) for the linear momentum is used in expression (65b) to give:

$$
V_{a} \frac{d \boldsymbol{F}_{a}}{d t}=\sum_{b \in \Lambda_{a}} \frac{1}{2 \rho_{0}}\left(\boldsymbol{p}_{a}+\boldsymbol{p}_{b}\right) \otimes \boldsymbol{C}_{a b}+\sum_{\gamma \in \Gamma_{a}^{B}} \frac{1}{\rho_{0}}\left(\boldsymbol{p}_{a}^{\gamma} \otimes \boldsymbol{N}_{\gamma}\right) \frac{A_{\gamma}}{3} .
$$

It can be clearly seen that the above equation updates the deformation gradient $\boldsymbol{F}_{a}$ using a discrete gradient of the velocities. This will ensure the satisfaction of the involutions (3) in a discrete manner [84].

Given an edge $a b$, its normalised area vector $\boldsymbol{N}_{a b}$ and the reconstructed values of the fluxes at its two sides (namely, $\boldsymbol{p}^{-,+}$and $\boldsymbol{P}^{-,+}$), an acoustic Riemann solver can be derived via the Rankine-Hugoniot jump conditions. This will provide two contact fluxes $\boldsymbol{p}^{C}$ and $\boldsymbol{t}^{C}$ to be used in computing the nunerical fluxes [3, 41].

To achieve this, the contact fluxes are decomposed into their normal and 
tangential components. The former relates to frictionless contact, whereas the latter associates with infinite friction contact. Given the redundant character of the system (1), only the jump condition for the linear momentum (equation (7a)) is considered.

In the case of frictionless contact, the generated shock waves will travel with volumetric wave speed $c_{p}$. The normal components of momentum and traction after contact can be written as

$$
\begin{aligned}
& c_{p}^{-}\left(p_{n}^{-}-p_{n}^{C}\right)=t_{n}^{-}-t_{n}^{C}, \\
& c_{p}^{+}\left(p_{n}^{+}-p_{n}^{C}\right)=-\left(t_{n}^{+}-t_{n}^{C}\right) .
\end{aligned}
$$

After some algebraic manipulations, equation (67) gives

$$
\begin{aligned}
p_{n}^{C} & =\frac{c_{p}^{-} p_{n}^{-}+c_{p}^{+} p_{n}^{+}}{c_{p}^{-}+c_{p}^{+}}+\frac{t_{n}^{+}-t_{n}^{-}}{c_{p}^{-}+c_{p}^{+}}, \\
t_{n}^{C} & =\frac{c_{p}^{-} c_{p}^{+}}{c_{p}^{-}+c_{p}^{+}}\left(\frac{t_{n}^{-}}{c_{p}^{-}}+\frac{t_{n}^{+}}{c_{p}^{+}}\right)+\frac{c_{p}^{-} c_{p}^{+}}{c_{p}^{-}+c_{p}^{+}}\left(p_{n}^{+}-p_{n}^{-}\right) .
\end{aligned}
$$

Here, $p_{n}^{-}$and $p_{n}^{+}$denote the left and right normal components of the linear momentum before contact, that is, $p_{n}^{-,+}=\boldsymbol{p}^{-,+} \cdot \boldsymbol{n}$. Analogously, $t_{n}^{-,+}$describe the normal components of the traction vector before contact, that is, $t_{n}^{-,+}=$ $\boldsymbol{n} \cdot\left(\boldsymbol{P}^{-,+} \boldsymbol{N}\right)$. Note that the surface normal is defined outwards for the $(-)$ body and inwards for the $(+)$ body (see Figure 3 ).

Additional shock waves will propogate with shear wave speed $c_{s}$ under infinite friction contact. An analogous derivation can now be followed for the tangential components of the traction and linear momentum:

$$
\begin{aligned}
c_{s}^{-}\left(\boldsymbol{p}_{t}^{-}-\boldsymbol{p}_{n}^{C}\right) & =\boldsymbol{t}_{t}^{-}-\boldsymbol{t}_{t}^{C}, \\
c_{s}^{+}\left(\boldsymbol{p}_{t}^{+}-\boldsymbol{p}_{n}^{C}\right) & =-\left(\boldsymbol{t}_{t}^{+}-\boldsymbol{t}_{t}^{C}\right) .
\end{aligned}
$$

The tangential contact fluxes can then be obtained:

$$
\begin{aligned}
\boldsymbol{p}_{t}^{C} & =\frac{c_{s}^{-} \boldsymbol{p}_{t}^{-}+c_{s}^{+} \boldsymbol{p}_{t}^{+}}{c_{s}^{-}+c_{s}^{+}}+\frac{\boldsymbol{t}_{t}^{+}-\boldsymbol{t}_{t}^{-}}{c_{s}^{-}+c_{s}^{+}} \\
\boldsymbol{t}_{t}^{C} & =\frac{c_{s}^{-} c_{s}^{+}}{c_{s}^{-}+c_{s}^{+}}\left(\frac{\boldsymbol{t}_{t}^{-}}{c_{s}^{-}}+\frac{\boldsymbol{t}_{t}^{+}}{c_{s}^{+}}\right)+\frac{c_{s}^{-} c_{s}^{+}}{c_{s}^{-}+c_{s}^{+}}\left(\boldsymbol{p}_{t}^{+}-\boldsymbol{p}_{t}^{-}\right)
\end{aligned}
$$


With the aid of above expressions (68) and (70), the complete contact momentum and traction vectors are defined by

$$
\boldsymbol{p}^{C}=\boldsymbol{p}_{t}^{C}+p_{n}^{C} \boldsymbol{n} ; \quad \boldsymbol{t}^{C}=\boldsymbol{t}_{t}^{C}+t_{n}^{C} \boldsymbol{n} .
$$

The spatial unit outward normal is defined as

$$
\boldsymbol{n}=\frac{\left[\frac{1}{2}\left(\boldsymbol{F}_{a}+\boldsymbol{F}_{b}\right)\right]^{-T} \boldsymbol{N}}{\left\|\left[\frac{1}{2}\left(\boldsymbol{F}_{a}+\boldsymbol{F}_{b}\right)\right]^{-T} \boldsymbol{N}\right\|},
$$

where the central difference approximation for $\boldsymbol{F}$ is used. The linear reconstruction of linear momentum and first Piola at both sides of the interface, namely $\boldsymbol{p}^{-,+}$and $\boldsymbol{P}^{-,+}$, can be easily obtained with the aid of (63).

\section{Time integration}

The spatial semidiscretisation leaves a system of Ordinary Differential Equations (ODE's) in time for each node $a$ written as,

$$
V_{a} \frac{d \mathcal{U}_{a}}{d t}=-\mathcal{R}_{a}\left(\mathcal{U}_{a}, t\right)
$$

where $\boldsymbol{\mathcal { R }}_{a}\left(\boldsymbol{U}_{a}, t\right)$ represents the right hand side of the discretised system (see (65a), (65c), (65d), and (66)) at node $a$.

Equation (73) is discretised using a Total Variation Diminishing RungeKutta (TVD-RK) time integrator as proposed by Shu and Osher [85, 86]. The Runge-Kutta method computes the solution at time step $t^{n+1}$ from the solution at time step $t^{n}$ as

$$
\begin{aligned}
\mathcal{U}_{a}^{*} & =\mathcal{U}_{a}^{n}-\Delta t \boldsymbol{R}_{a}\left(\mathcal{U}_{a}^{n}, t^{n}\right) \\
\mathcal{U}_{a}^{* *} & =\mathcal{U}_{a}^{*}-\Delta t \boldsymbol{R}_{a}\left(\mathcal{U}_{a}^{*}, t^{n+1}\right) \\
\mathcal{U}_{a}^{n+1} & =\frac{1}{2}\left(\mathcal{U}_{a}^{n}+\mathcal{U}_{a}^{* *}\right)
\end{aligned}
$$

The maximum time step is governed by a standard Courant-Friedrichs-Lewy (CFL) condition [76, 87] given as

$$
\Delta t \leq \alpha_{C F L} \min _{a}\left(\frac{h_{a}}{c_{p, a}^{n}}\right),
$$


where $h_{a}$ and $c_{p, a}^{n}$ are the minimum length and the volumetric wave speed (56) associated to node $a$ at the reference domain and $\alpha_{C F L}$ is the CFL stability number. For a linear advection problem, the combination of a Finite Volume technique and a two-step Runge-Kutta time integrator has a stability region given by $0 \leq \alpha_{C F L} \leq 1$ [76]. For the test cases presented in this paper, a value of $\alpha_{C F L}=0.4$ has been chosen, which is sufficient to ensure accuracy and stability.

In addition, the displacements are integrated in time using the trapezoidal rule as,

$$
\boldsymbol{x}_{a}^{n+1}=\boldsymbol{x}_{a}^{n}+\frac{\Delta t}{2}\left(\boldsymbol{v}_{a}^{n}+\boldsymbol{v}_{a}^{n+1}\right)
$$

\section{Discrete angular momentum conserving algorithm}

As presented in $[1,3,40]$, a Lagrangian correction procedure for a set of internal forces $\boldsymbol{f}_{k}=\boldsymbol{t}^{C}\left\|\boldsymbol{C}_{a b}\right\|$ needs to be carried out to ensure the preservation of angular momentum. To achieve this, a minimization procedure will be carried out such that the internal forces are minimally modified while ensuring the discrete conservation of angular momentum. Moreover, given the conservative properties of the numerical scheme, the use of a modified contact traction will not affect the conservation of linear momentum and total energy. The procedure is detailed in what follows.

In the absence of external tractions, the conservation of the discrete angular momentum after a time step can be written as

$$
\sum_{a=1}^{N_{\text {nodes }}} \boldsymbol{x}_{a}^{n+1} \times m_{a} \boldsymbol{v}_{a}^{n+1}-\sum_{a=1}^{N_{\text {nodes }}} \boldsymbol{x}_{a}^{n} \times m_{a} \boldsymbol{v}_{a}^{n}=\mathbf{0} .
$$

By taking into account the trapezoidal rule for the time integration of the positions (see equation (76)), the above equation can be rewritten as

$$
\sum_{a=1}^{N_{\text {nodes }}} \boldsymbol{x}_{a}^{n+1 / 2} \times m_{a} \Delta \boldsymbol{v}_{a}=\mathbf{0} ; \quad \Delta \boldsymbol{v}_{a}=\boldsymbol{v}_{a}^{n+1}-\boldsymbol{v}_{a}^{n} ; \quad \boldsymbol{x}_{a}^{n+1 / 2}=\boldsymbol{x}_{a}^{n}+\frac{\Delta t}{2} \boldsymbol{v}_{a}^{n}
$$

Considering the TVD Runge-Kutta time integration as presented in equation (74), the velocity increment reads 


$$
\Delta \boldsymbol{v}_{a}=-\frac{\Delta t}{2 \rho_{0}}\left(\boldsymbol{\mathcal { R }}_{a}^{n}\left(\boldsymbol{p}_{a}^{n}, t^{n}\right)+\boldsymbol{\mathcal { R }}_{a}^{*}\left(\boldsymbol{p}_{a}^{*}, t^{*}\right)\right),
$$

where $\boldsymbol{\mathcal { R }}_{a}\left(\boldsymbol{p}_{a}, t\right)$ corresponds to the right hand side of the nodal linear momentum (65a). Substituting (79) into (78), the following equation is obtained

$$
\sum_{e=1}^{N_{\text {nodes }}} \boldsymbol{x}_{a}^{n+1 / 2} \times m_{a}\left(-\frac{\Delta t}{2 \rho_{0}}\left(\boldsymbol{\mathcal { R }}_{a}^{n}\left(\boldsymbol{p}_{a}^{n}, t^{n}\right)+\boldsymbol{\mathcal { R }}_{a}^{*}\left(\boldsymbol{p}_{a}^{*}, t^{*}\right)\right)\right)=\mathbf{0} .
$$

Sufficient conditions to satisfy expression (80) is described as

$$
\sum_{a=1}^{N_{\text {nodes }}} \boldsymbol{x}_{a}^{n+1 / 2} \times m_{a} \boldsymbol{\mathcal { R }}_{a}^{\alpha}\left(\boldsymbol{p}_{a}^{\alpha}, t^{\alpha}\right)=\mathbf{0},
$$

where the equation above needs to be fulfilled at each stage of the RungeKutta time integrator (i.e., $\forall \alpha \in\{n, *\}$ ). Substituting the right hand side of equation (65a) into (81) and omitting the time superindex for simplicity, to give:

$$
\sum_{a=1}^{N_{\text {nodes }}} \boldsymbol{x}_{a} \times \frac{m_{a}}{\rho_{0} V_{a}}\left(\sum_{\alpha \in \Lambda_{a}} \boldsymbol{t}^{C}\left\|\boldsymbol{C}_{a b}\right\|+\sum_{\gamma \in \Gamma_{a}^{B}} \hat{\boldsymbol{t}}_{a}^{\gamma} \frac{A^{\gamma}}{3}\right)=\mathbf{0} .
$$

Assuming a free boundary traction (that could otherwise contribute to an external torque) and rearranging the term into a summation over edges:

$$
\sum_{k=1}^{N_{e d_{i n t}}} \boldsymbol{f}_{k} \times \Delta \boldsymbol{x}=\mathbf{0} ; \quad \Delta \boldsymbol{x}=\boldsymbol{x}_{b}^{n+1 / 2}-\boldsymbol{x}_{a}^{n+1 / 2}
$$

where $\boldsymbol{N}_{a b}=-\boldsymbol{N}_{b a}$ and $\boldsymbol{f}_{k}=\boldsymbol{t}^{C}\left\|\boldsymbol{C}_{a b}\right\|$ is the force related to edge $k$.

A Lagrangian minimisation procedure is used to obtain a modified set of internal forces $\hat{\boldsymbol{f}}_{k}$. This can be achieved by minimising the following functional

$$
\Pi_{f}\left(\hat{\boldsymbol{f}}_{k}, \boldsymbol{\lambda}_{f}\right)=\left(\frac{1}{2} \sum_{k=1}^{N_{e d_{i n t}}}\left(\hat{\boldsymbol{f}}_{k}-\boldsymbol{f}_{k}\right) \cdot\left(\hat{\boldsymbol{f}}_{k}-\boldsymbol{f}_{k}\right)\right)+\boldsymbol{\lambda}_{f} \cdot \sum_{k=1}^{N_{e d_{i n t}}} \hat{\boldsymbol{f}}_{k} \times \Delta \boldsymbol{x}_{k}
$$


After some algebraic manipulations, a modified set of internal forces $\hat{\boldsymbol{f}}_{k}$ is obtained as

$$
\hat{\boldsymbol{f}}_{k}=\boldsymbol{f}_{k}+\boldsymbol{\lambda}_{f} \times \Delta \boldsymbol{x}_{k} .
$$

The Lagrange multiplier $\boldsymbol{\lambda}_{f}$ is the solution of the following $3 \times 3$ system of equations

$$
\boldsymbol{K}_{f} \boldsymbol{\lambda}_{f}=\boldsymbol{b}_{f},
$$

where

$$
\boldsymbol{K}_{f}=\sum_{k=1}^{N_{e d_{i n t}}}\left(\Delta \boldsymbol{x}_{k} \cdot \Delta \boldsymbol{x}_{k}\right) \boldsymbol{I}-\Delta \boldsymbol{x}_{k} \otimes \Delta \boldsymbol{x}_{k} ; \quad \boldsymbol{b}_{f}=\sum_{k=1}^{N_{e d_{i n t}}} \boldsymbol{f}_{k} \times \Delta \boldsymbol{x}_{k} .
$$

\section{Solution procedure}

The algorithm 6.1 summarises the solution procedure of the upwind vertex centred FVM (see section 3) in conjunction with the one-step two-stage 
explicit TVD-RK time integrator (see section 4):

Algorithm 6.1: Evaluation of $\mathcal{U}_{n+1}\left(\mathcal{U}_{n}\right)$

(1) GIVEN $\mathcal{U}_{a}^{n}=\left(\boldsymbol{p}_{a}^{n}, \boldsymbol{F}_{a}^{n}, J_{a}^{n}, E_{a}^{n}\right)^{T}, \boldsymbol{x}_{a}^{n}$

(2) LOOP over Runge-Kutta stages (to compute $\mathcal{U}_{a}^{*}, \boldsymbol{U}_{a}^{* *}$ )

(2.1)LOOP over edges $k(a b)$

$$
\begin{aligned}
& \boldsymbol{G}_{a}^{\boldsymbol{p}}:=\boldsymbol{G}_{a}^{\boldsymbol{p}}+\frac{1}{2 V_{a}}\left(\boldsymbol{p}_{a}+\boldsymbol{p}_{b}\right) \otimes \boldsymbol{C}_{a b} \\
& \boldsymbol{G}_{a}^{\boldsymbol{P}}:=\boldsymbol{G}_{a}^{\boldsymbol{P}}+\frac{1}{2 V_{a}}\left(\boldsymbol{P}_{a}+\boldsymbol{P}_{b}\right) \otimes \boldsymbol{C}_{a b}
\end{aligned}
$$

(proceed equivalently for node $b$ )

(2.2)LOOP over boundary faces $\gamma(a b c)$

$\boldsymbol{G}_{a}^{p}:=\boldsymbol{G}_{a}^{p}+\frac{1}{V_{a}}\left(\boldsymbol{p}_{a}^{\gamma} \otimes \boldsymbol{N}_{\gamma}\right) \frac{A_{\gamma}}{3}$

$\boldsymbol{G}_{a}^{\boldsymbol{P}}:=\boldsymbol{G}_{a}^{\boldsymbol{P}}+\frac{1}{V_{a}}\left(\boldsymbol{P}_{a}^{\gamma} \otimes \boldsymbol{N}_{\gamma}\right) \frac{A_{\gamma}}{3}$

(proceed equivalently for nodes $b, c$ )

(2.3)LOOP over edges $k(a b)$

$$
\begin{aligned}
& \boldsymbol{f}_{k}^{n}=\boldsymbol{t}^{C, n}\left\|\boldsymbol{C}_{a b}\right\| \\
& \boldsymbol{b}_{f}:=\boldsymbol{b}_{f}+\boldsymbol{f}_{k}^{n} \times \Delta \boldsymbol{x}_{k}^{n+1 / 2} \\
& \boldsymbol{K}_{f}:=\boldsymbol{K}_{f}+\left(\Delta \boldsymbol{x}_{k}^{n+1 / 2} \cdot \Delta \boldsymbol{x}_{k}^{n+1 / 2}\right) \boldsymbol{I}-\Delta \boldsymbol{x}_{k}^{n+1 / 2} \otimes \Delta \boldsymbol{x}_{k}^{n+1 / 2}
\end{aligned}
$$

(2.4)COMPUTE $\boldsymbol{\lambda}_{f}=\boldsymbol{K}_{f}^{-1} \boldsymbol{b}_{f}$

(2.5)LOOP over edges $k(a b)$

$$
\begin{aligned}
& \hat{\boldsymbol{f}}_{k}^{n}=\boldsymbol{f}_{k}^{n}+\boldsymbol{\lambda}_{f} \times \Delta \boldsymbol{x}_{k}^{n+1 / 2} \\
& \mathcal{R}_{\boldsymbol{p}_{a}}^{n}:=\boldsymbol{\mathcal { R }}_{\boldsymbol{p}_{a}}^{n}+\hat{\boldsymbol{f}}_{k}^{n} ; \quad \boldsymbol{\mathcal { R }}_{\boldsymbol{F}_{a}}^{n}:=\boldsymbol{\mathcal { R }}_{\boldsymbol{F}_{a}}^{n}+\frac{1}{2}\left(\boldsymbol{v}_{a}^{n}+\boldsymbol{v}_{b}^{n}\right) \otimes \boldsymbol{C}_{a b} \\
& \mathcal{R}_{J_{a}}^{n}:=\mathcal{R}_{J_{a}}^{n}+\left(\boldsymbol{H}_{\boldsymbol{F}_{a}^{n}}^{T} \boldsymbol{v}^{C, n}\right) \cdot \boldsymbol{C}_{a b} \\
& \mathcal{R}_{E_{a}}^{n}:=\mathcal{R}_{E_{a}}^{n}+\left(\boldsymbol{t}^{C, n} \cdot \boldsymbol{v}^{C, n}\right)\left\|\boldsymbol{C}_{a b}\right\|
\end{aligned}
$$

(proceed equivalently for node $b$ )

(2.6)LOOP over boundary faces $\gamma(a b c)$ according to B.C.

$$
\begin{aligned}
& \mathcal{R}_{\boldsymbol{p}_{a}}^{n}:=\mathcal{R}_{\boldsymbol{p}_{a}}^{n}+\boldsymbol{t}_{a}^{\gamma} \frac{A_{\gamma}}{3} ; \quad \mathcal{R}_{\boldsymbol{F}_{a}}^{n}:=\mathcal{R}_{\boldsymbol{F}_{a}}^{n}+\left(\boldsymbol{v}_{a}^{\gamma} \otimes \boldsymbol{N}_{\gamma}\right) \frac{A_{\gamma}}{3} \\
& \mathcal{R}_{J_{a}}^{n}:=\mathcal{R}_{J_{a}}^{n}+\left(\boldsymbol{H}_{\boldsymbol{F}_{a}^{n}}^{T} \boldsymbol{v}_{a}^{\gamma}\right) \cdot \boldsymbol{N}_{\gamma} \frac{A_{\gamma}}{3} \\
& \mathcal{R}_{E_{a}}^{n}:=\mathcal{R}_{E_{a}}^{n}+\left(\boldsymbol{t}_{a}^{\gamma} \cdot \boldsymbol{v}_{a}^{\gamma}\right) \frac{A_{\gamma}}{3}
\end{aligned}
$$

(proceed equivalently for nodes $b, c$ )

(2.7)UPDATE conservation variables at stage

$$
\mathcal{U}_{a}^{*}=\mathcal{U}_{a}^{n}+\frac{1}{V_{a}} \boldsymbol{\mathcal { R }}_{\mathcal{U}_{a}}^{n}
$$

(2.8)EVALUATE $\boldsymbol{P}_{a}^{*}=\boldsymbol{P}\left(\boldsymbol{F}_{a}^{*}, J_{a}^{*}, E_{a}^{*}\right)$ (only after stage 1)

(2.9)APPLY strong BC

(3) UPDATE conservation variables and positions

$$
\boldsymbol{U}_{a}^{n+1}=\frac{1}{2}\left(\mathcal{U}_{a}^{n}+\mathcal{U}_{a}^{* *}\right) ; \quad \boldsymbol{x}_{a}^{n+1}=\boldsymbol{x}^{n}+\frac{\Delta t}{2}\left(\boldsymbol{v}_{a}^{n}+\boldsymbol{v}_{a}^{n+1}\right)
$$

(4) EVALUATE $\boldsymbol{P}_{a}^{n+1}=\boldsymbol{P}\left(\boldsymbol{F}_{a}^{n+1}, J_{a}^{n+1}, E_{a}^{n+1}\right)$

(5) APPLY strong BC 


\section{Numerical examples}

\subsection{Low dispersion cube}

This example is a three dimensional extension of the swinging plate presented in $[3,40]$ and was previously assessed in $[1,5]$. A cube of unit side has symmetric boundary conditions (roller supports) at the faces $X=0, Y=0$ and $Z=0$ and skew-symmetric boundary conditions (restricted tangential displacement) at the faces $X=1, Y=1$ and $Z=1$ (see Figure 1 for a schematic representation of the different types of boundary conditions). For small deformations, the problem has an analytical displacement field of the form

$$
\boldsymbol{u}(\boldsymbol{X}, t)=U_{0} \cos \left(\frac{\sqrt{3}}{2} c_{d} \pi t\right)\left[\begin{array}{l}
A \sin \left(\frac{\pi X_{1}}{2}\right) \cos \left(\frac{\pi X_{2}}{2}\right) \cos \left(\frac{\pi X_{3}}{2}\right) \\
B \cos \left(\frac{\pi X_{1}}{2}\right) \sin \left(\frac{\pi X_{2}}{2}\right) \cos \left(\frac{\pi X_{3}}{2}\right) \\
C \cos \left(\frac{\pi X_{1}}{2}\right) \cos \left(\frac{\pi X_{2}}{2}\right) \sin \left(\frac{\pi X_{3}}{2}\right)
\end{array}\right] .
$$

Parameters $\{A, B, C\}$ are user-defined arbitrary constants such that $A+$ $B+C=0$ ensuring no contribution from volumetric deformation. For values of $U_{0}$ below $0.001 \mathrm{~m}$, the solution can be considered to be linear and the closed-form expression (86) holds. The problem is initialised with a known deformation gradient field $\boldsymbol{F}(\boldsymbol{X}, 0)=\boldsymbol{I}+\boldsymbol{\nabla}_{0} \boldsymbol{u}$, and the initial Jacobian $J(\boldsymbol{X}, 0)=\operatorname{det} \boldsymbol{F}$ is similarly obtained. In this particular example, a linear elastic material is chosen with a Poisson's ratio of $\nu=(1-\mu / \kappa) / 2=0.45$, Young's modulus $E=1.7 \times 10^{7} \mathrm{~Pa}$ and density $\rho_{0}=1.1 \times 10^{3} \mathrm{~kg} / \mathrm{m}^{3}$. The solution parameters are set as $A=1, B=1, C=-2$ and $U_{0}=5 \times 10^{-4} \mathrm{~m}$.

The main aim of this example is to assess the convergence behaviour of the upwind FVM. Tables 1 to 4 show a global convergence analysis (e.g. $L^{1}$ and $L^{2}$ norm errors) of the linear momentum $\boldsymbol{p}$ and the first Piola $\boldsymbol{P}$ as compared to the analytical solution given in (86). Figure 4 shows these results graphically. As expected, the upwind method shows a second order convergence pattern with a lower translation error that that of JamesonSchmidt-Turkel (JST) algorithm. Given the same target error for all norms and variables, the upwind method requires less computational time compared to the JST algorithm, as shown in Figure (5).

\subsection{One dimensional Sod shock tube}

This example was first presented in [88] and later used by many authors $[55,60,89]$. The example is designed to assess the shock capturing capabilities of the method. A gas, governed by the ideal gas law (41), remains at rest 
Table 1: Low dispersion cube. Numerical values for the relative error of the linear momentum as compared to the exact solution, measured with the $L^{1}$ norm. Comparison between the upwind and JST FVM. Convergence rate calculated using the results of the two finest meshes

\begin{tabular}{cccc}
\hline$h$ & JST, $\kappa^{(4)}=1 / 128$ & JST, $\kappa^{(4)}=1 / 256$ & $\mathrm{UW}$ \\
\hline \hline $1 / 3$ & $2.123 \times 10^{-1}$ & $1.894 \times 10^{-1}$ & $9.912 \times 10^{-2}$ \\
$1 / 6$ & $9.891 \times 10^{-2}$ & $7.597 \times 10^{-2}$ & $1.871 \times 10^{-2}$ \\
$1 / 12$ & $3.433 \times 10^{-2}$ & $2.294 \times 10^{-2}$ & $3.022 \times 10^{-3}$ \\
$1 / 24$ & $9.829 \times 10^{-3}$ & $6.159 \times 10^{-3}$ & $7.605 \times 10^{-4}$ \\
\hline conv. rate & 1.805 & 1.897 & 1.991 \\
\hline
\end{tabular}

Table 2: Low dispersion cube. Numerical values for the relative error of the linear momentum as compared to the exact solution, measured with the $L^{2}$ norm. Comparison between the upwind and JST FVM. Convergence rate calculated using the results of the two finest meshes

\begin{tabular}{cccc}
\hline$h$ & JST, $\kappa^{(4)}=1 / 128$ & $\mathrm{JST}, \kappa^{(4)}=1 / 256$ & $\mathrm{UW}$ \\
\hline \hline $1 / 3$ & $2.236 \times 10^{-1}$ & $1.965 \times 10^{-1}$ & $1.000 \times 10^{-1}$ \\
$1 / 6$ & $1.003 \times 10^{-1}$ & $7.565 \times 10^{-2}$ & $1.939 \times 10^{-2}$ \\
$1 / 12$ & $3.409 \times 10^{-2}$ & $2.298 \times 10^{-2}$ & $3.284 \times 10^{-3}$ \\
$1 / 24$ & $1.056 \times 10^{-2}$ & $6.656 \times 10^{-3}$ & $8.232 \times 10^{-4}$ \\
\hline conv. rate & 1.691 & 1.788 & 1.996 \\
\hline
\end{tabular}


Table 3: Low dispersion cube. Numerical values for the relative error of the stress as compared to the exact solution, measured with the $L^{1}$ norm. Comparison between the upwind and JST FVM. Convergence rate calculated using the results of the two finest meshes

Error values stress, $L^{1}$ norm

\begin{tabular}{cccc}
\hline$h$ & JST, $\kappa^{(4)}=1 / 128$ & JST, $\kappa^{(4)}=1 / 256$ & UW \\
\hline \hline $1 / 3$ & $2.200 \times 10^{-3}$ & $1.964 \times 10^{-3}$ & $1.028 \times 10^{-3}$ \\
$1 / 6$ & $9.617 \times 10^{-4}$ & $7.386 \times 10^{-4}$ & $1.819 \times 10^{-4}$ \\
$1 / 12$ & $3.295 \times 10^{-4}$ & $2.201 \times 10^{-4}$ & $2.900 \times 10^{-5}$ \\
$1 / 24$ & $9.399 \times 10^{-5}$ & $5.889 \times 10^{-5}$ & $7.272 \times 10^{-6}$ \\
\hline conv. rate & 1.810 & 1.902 & 1.996 \\
\hline
\end{tabular}

Table 4: Low dispersion cube. Numerical values for the relative error of the stress as compared to the exact solution, measured with the $L^{2}$ norm. Comparison between the upwind and JST FVM. Convergence rate calculated using the results of the two finest meshes

Error values stress, $L^{2}$ norm

\begin{tabular}{cccc}
\hline$h$ & JST, $\kappa^{(4)}=1 / 128$ & JST, $\kappa^{(4)}=1 / 256$ & UW \\
\hline \hline $1 / 3$ & $2.555 \times 10^{-1}$ & $2.271 \times 10^{-1}$ & $1.912 \times 10^{-1}$ \\
$1 / 6$ & $1.302 \times 10^{-1}$ & $1.018 \times 10^{-1}$ & $6.280 \times 10^{-2}$ \\
$1 / 12$ & $5.597 \times 10^{-2}$ & $3.781 \times 10^{-2}$ & $1.797 \times 10^{-2}$ \\
$1 / 24$ & $1.820 \times 10^{-2}$ & $1.156 \times 10^{-2}$ & $5.173 \times 10^{-3}$ \\
\hline conv. rate & 1.621 & 1.709 & 1.800 \\
\hline
\end{tabular}




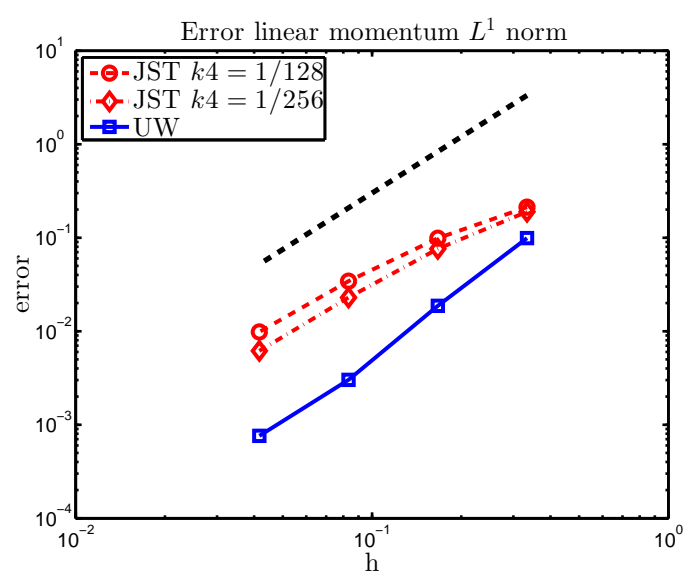

(a)

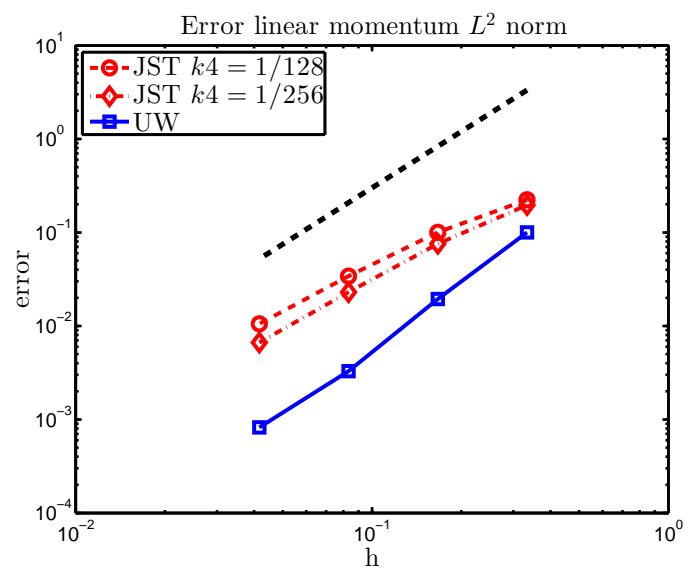

(c)

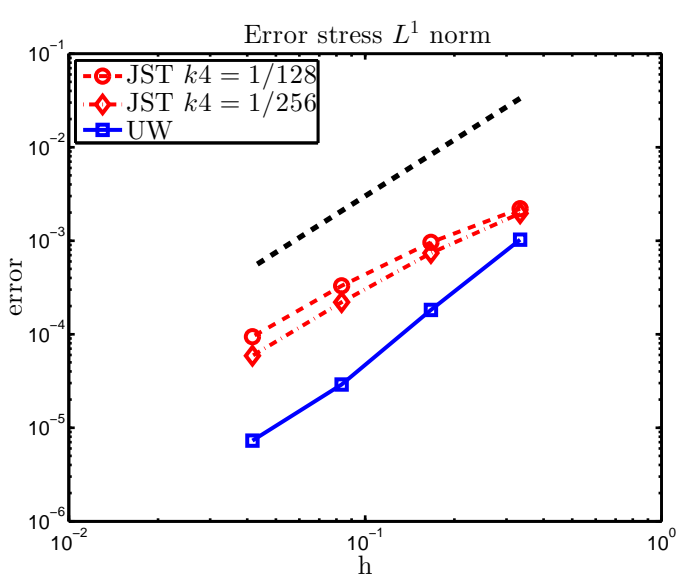

(b)

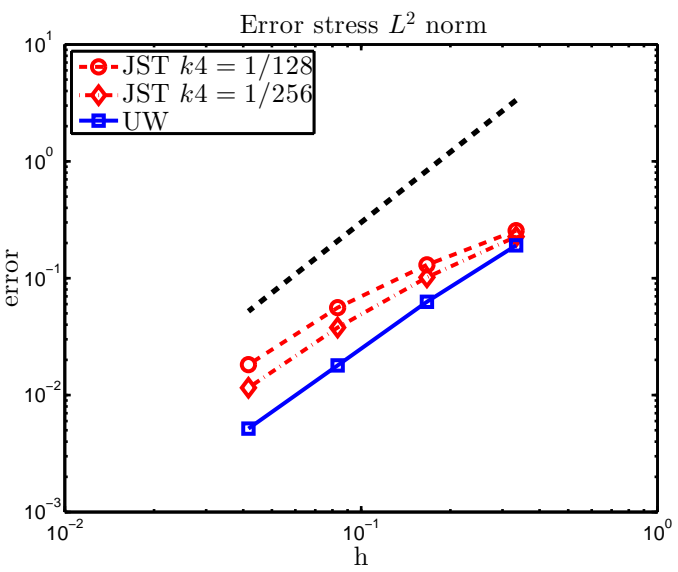

(d)

Figure 4: Low dispersion cube. Convergence of the $L^{1}$ and $L^{2}$ norms for linear momentum and stress at time $t=0.004 \mathrm{~s}$. Comparison of the order of convergence between the upwind and the JST FVM. Linear elastic material with Poisson's ratio $\nu=(1-\mu / \kappa) / 2=0.45$, Young's modulus $E=1.7 \times$ $10^{7} \mathrm{~Pa}$ and density $\rho_{0}=1.1 \times 10^{3} \mathrm{~kg} / \mathrm{m}^{3}$. 


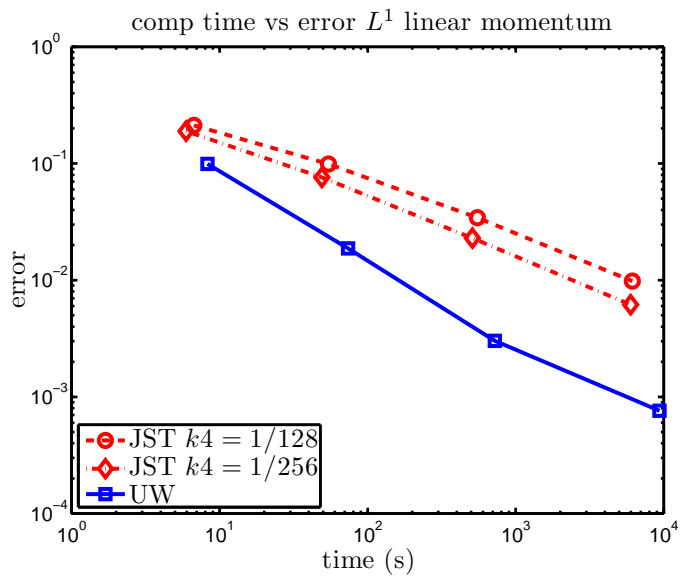

(a)

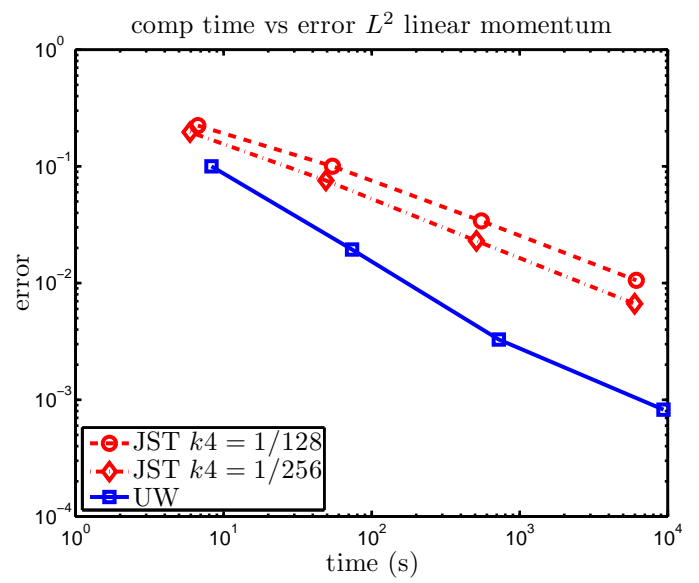

(c)

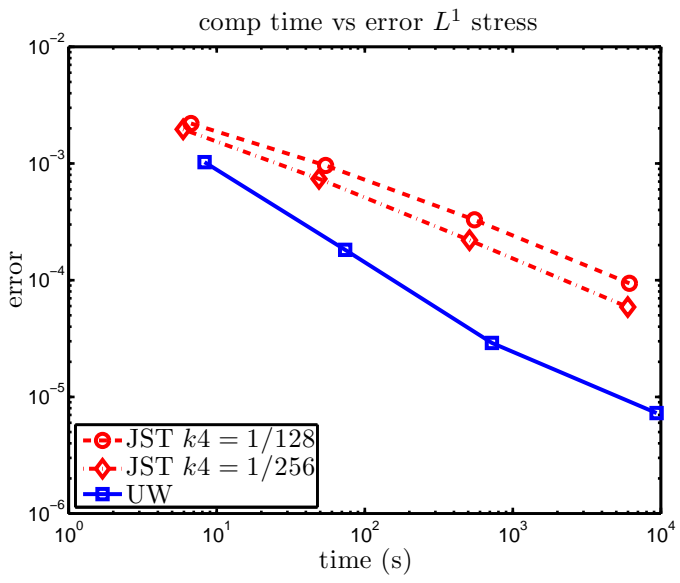

(b)

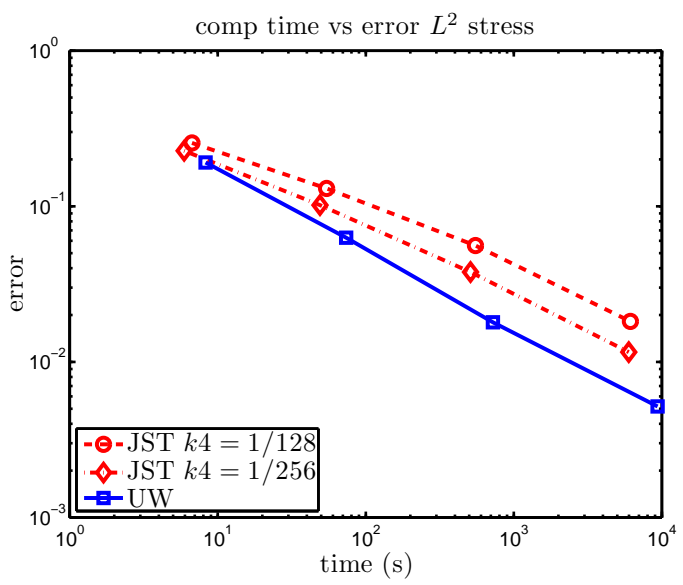

(d)

Figure 5: Low dispersion cube. Computational time against $L^{1}$ and $L^{2}$ norm errors for linear momentum and stress at time $t=0.004 \mathrm{~s}$. Comparison between the upwind and the JST FVM. Linear elastic material with Poisson's ratio $\nu=(1-\mu / \kappa) / 2=0.45$, Young's modulus $E=1.7 \times 10^{7} \mathrm{~Pa}$ and density $\rho_{0}=1.1 \times 10^{3} \mathrm{~kg} / \mathrm{m}^{3}$. 


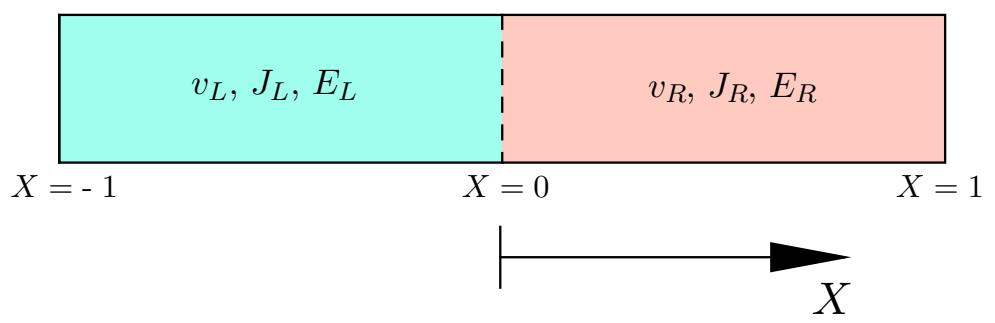

Figure 6: Setup shock tube.

inside a tube of $2 \mathrm{~m}$ length which is divided into two halves by a diaphragm (see Figure 6). The gas has a Grüneisen coefficient of $\Gamma_{0}=\frac{2}{5}$ and a density at the reference temperature of $\rho_{0}=1 \mathrm{~kg} / \mathrm{m}^{3}$. At time $t=0$, the gas on the left half side of the domain remains undeformed, whilst the gas on the right half side is expanded from a reference state where it was occupying one eighth of the current volume. Hence, the Jacobian of the deformation on the left half is $J_{L}=1$ whilst $J_{R}=8$ on the right half (or equivalently, $\rho_{L}=1 \mathrm{~kg} / \mathrm{m}^{3}$ and $\rho_{R}=0.125 \mathrm{~kg} / \mathrm{m}^{3}$ ). In addition, there is a jump in pressure between the left and right domain, $p_{L}=-1 \mathrm{~Pa}$ and $p_{R}=-0.1 \mathrm{~Pa}$, which can be imposed through a jump in the total energy $\left(E_{L}=2.5 \mathrm{~J}\right.$ and $\left.E_{R}=2 \mathrm{~J}\right)$. At time $t=0$, the diaphragm is removed allowing the gas particles of the whole domain interact with each other. The predicted solution is compared against an analytical solution [60] at a particular time $t=0.25 \mathrm{~s}$.

Figures 7 and 8 show the solution profiles for the velocity, Jacobian of the deformation, total energy and pressure against the analytical solution using a discretisation of 100 elements and $\alpha_{C F L}=0.4$. Figure 7 corresponds to the JST solution [1] by activating a tailor-made discontinuity switch (e.g. $\kappa^{(2)}=1 / 4$ and $\kappa^{(4)}=1 / 16$ ), whereas Figure 8 corresponds to the solution obtained with the upwind FVM imposing limiter reconstruction (without the need to tune stabilising parameters). It is clear that the upwind FVM with slope limiter provides a relatively better resolution in the vicinity of sharp spatial gradients.

\subsection{One dimensional Woodward-Colella blast test}

Similar to the previous Sod shock tube problem (see Section 7.2), this example [90] is a severe numerical test to assess the performance of a shock capturing algorithm in an extreme scenario. A shock tube has an ideal gas 
with material properties $\rho_{0}=1 \mathrm{~kg} / \mathrm{m}^{3}$ and $\Gamma_{0}=\frac{2}{5}$ (see Figure 6). The gas is initially at rest but has a rapid jump in pressure between the left and right domains. The pressure on the left domain is $p_{L}=-1000 \mathrm{~Pa}$, whilst on the right domain $p_{R}=-0.01 \mathrm{~Pa}$. These conditions can be imposed through the total energy, namely $E_{L}=2.5 \times 10^{3} \mathrm{~Pa}$ and $E_{R}=2.5 \times 10^{-2} \mathrm{~Pa}$. At time $t=0$, the diaphragm is opened and the solution allowed to evolve in time. Figure 9 shows the solutions obtained from upwind FVM at time $t=1.2 \times 10^{-2} \mathrm{~s}$, using 100 elements and $\alpha_{C F L}=0.4$, as compared to the analytical solutions [60]. The predicted solution is relatively smooth, but a small overshoot can be seen at the vicinity of the shock in three of the variables $(J, E$ and $p)$. This is most likely due to the fact that an acoustic Riemann solver is used. In order to improve the shock capturing capabilities of the scheme, a nonlinear Riemann solver should be implemented [60].

\subsection{Two dimensional Woodward-Colella blast test}

This example is an extension of the one dimensional Colella blast test presented in section 7.3. The one dimensional shock tube is solved using an axisymmetric domain (as it was done for the Noh test in [91]), and therefore the one dimensional analytical solution of [60] remains still valid. Figure 10 shows the setup of the problem. The solution is obtained using $2 \times 64 \times 64$ triangular elements and $\alpha_{C F L}=0.4$. A series of snapshots are shown in Figure 11 for four different variables at a particular time: velocity, Jacobian of the deformation, total energy and pressure. Figure 12 shows the spatial distribution of the same variables, but this time plot as a function of the radius. It can be clearly seen that the upwind FVM imposing suitable slope limiter is very efficient in this shock-dominated problem.

\subsection{Elastic vibration of a Beryllium plate}

The main aim of this example is to evaluate the dissipation properties of the method in the linear elastic regime [1,48, 92]. A plate of $6 \mathrm{~cm}$ length and $1 \mathrm{~cm}$ width is made of Beryllium [1,48] using a compressible Neo-Hookean material with properties $\rho_{0}=1845 \mathrm{~kg} / \mathrm{m}^{3}, E=3.1827 \times 10^{11} \mathrm{~Pa}$ and $\nu=$ 0.05390. The plate is free of constraints and has an initial velocity that excites the first flexural mode (see Figure 13) described by

$$
\boldsymbol{v}_{0}=\left(0, v\left(X_{1}\right)\right)^{T} \mathrm{~m} / \mathrm{s},
$$



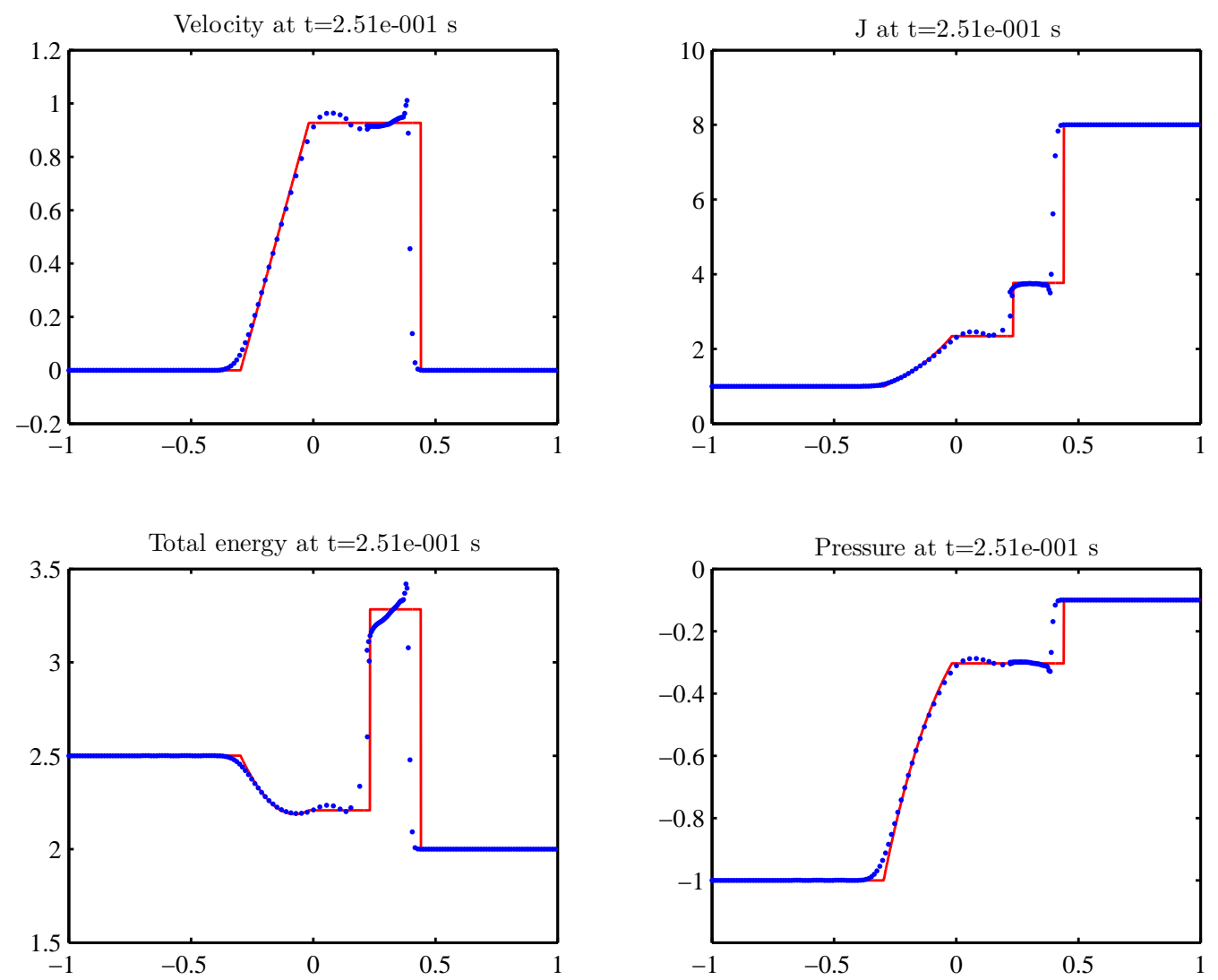

Figure 7: Sod's shock tube. Comparison of the profiles for the velocity, Jacobian of the deformation, total energy and pressure against the analytical solution at time $\mathrm{t}=0.25 \mathrm{~s}$. Ideal gas with $\Gamma_{0}=\frac{2}{5}$. Solutions obtained using the JST FVM with 100 elements imposing $\kappa^{(4)}=1 / 16$ and $\kappa^{(2)}=1 / 4$. Temporal discretisation using $\alpha_{C F L}=0.4$. 

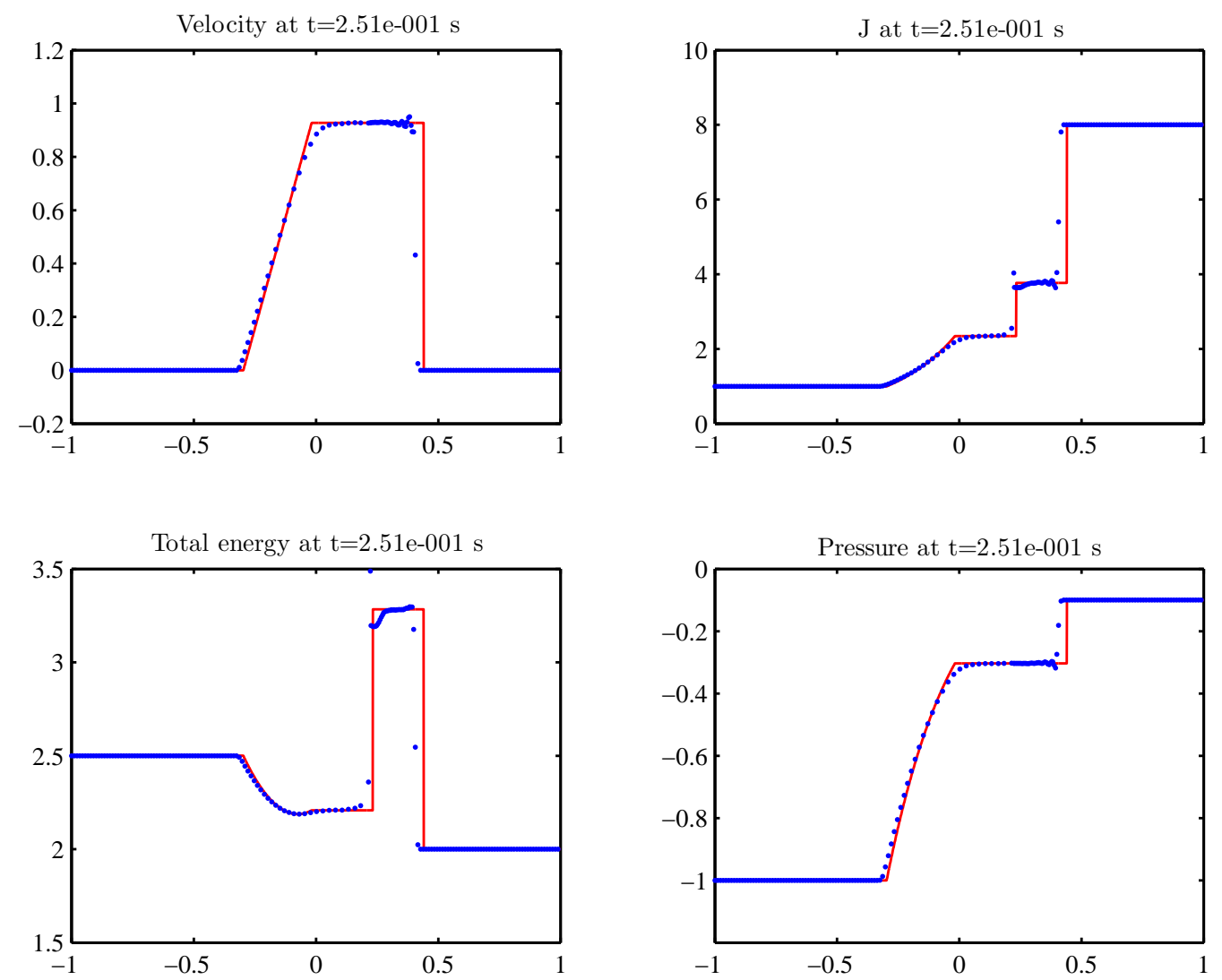

Figure 8: Sod's shock tube. Comparison of the profiles for the velocity, Jacobian of the deformation, total energy and pressure against the analytical solution at time $\mathrm{t}=0.25 \mathrm{~s}$. Ideal gas with $\Gamma_{0}=\frac{2}{5}$. Solutions obtained using the upwind FVM with 100 elements and Barth-Jespersen limiter. Temporal discretisation using $\alpha_{C F L}=0.4$. 

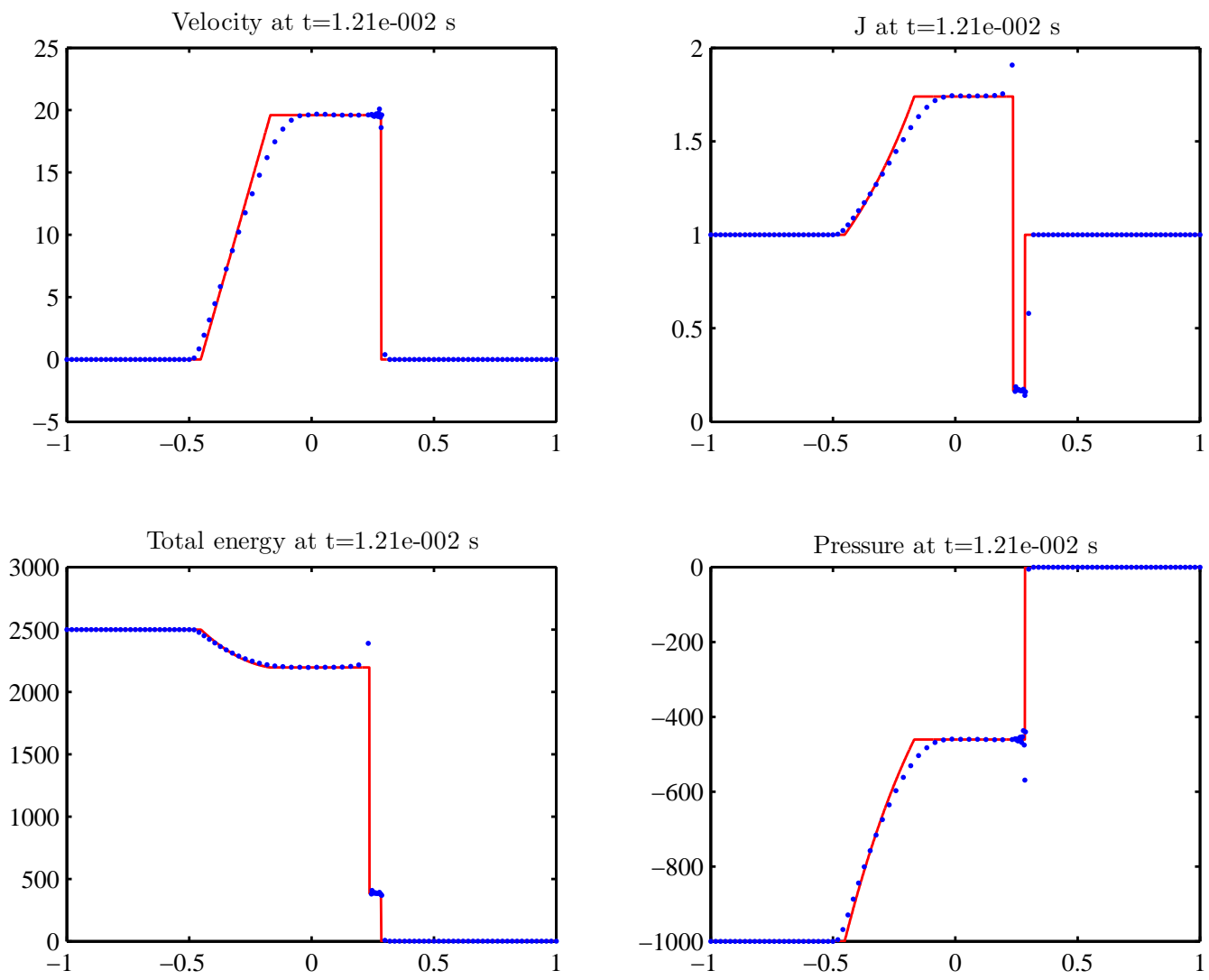

Figure 9: Woodward-Colella blast test. Comparison of the profiles for the velocity, Jacobian of the deformation, total energy and pressure against the analytical solution at time $\mathrm{t}=0.012 \mathrm{~s}$. Ideal gas with $\Gamma_{0}=\frac{2}{5}$. Solutions obtained using the upwind FVM with 100 elements and Barth-Jespersen limiter. Temporal discretisation using $\alpha_{C F L}=0.4$. 


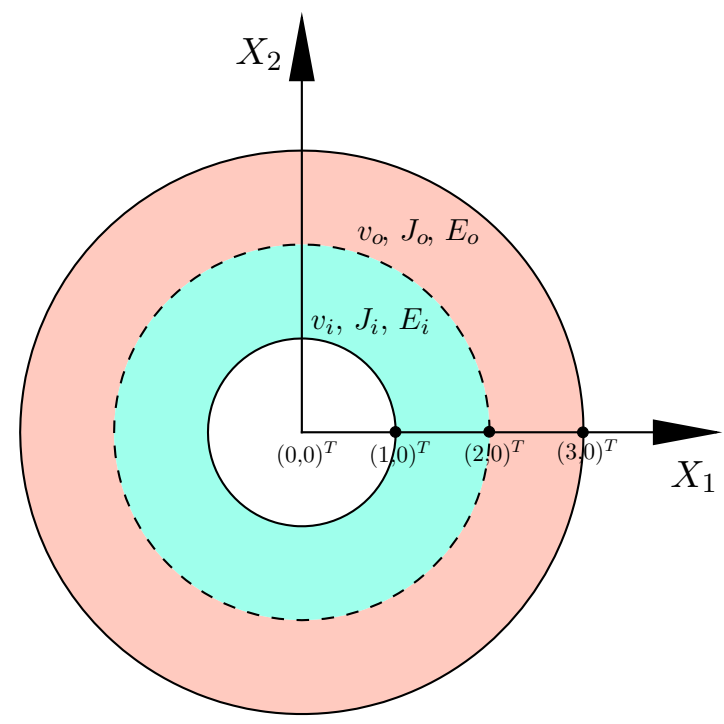

Figure 10: Setup for the axisymmetric Woodward-Colella blast test.

where $v\left(X_{1}\right)=A \omega\left[g_{1}(\sinh (\Omega l)+\sin (\Omega l))-g_{2}(\cosh (\Omega l)+\cos (\Omega l))\right]$. The parameters are given as $[48,92]$

$$
\begin{aligned}
& g_{1}=56.637, \quad g_{2}=57.646, \quad \omega=2.3597 \times 10^{5} \mathrm{~s}^{-1}, \quad A=4.3369 \times 10^{-5} \mathrm{~m} \\
& \Omega=78.834 \mathrm{~m}^{-1}, \quad l=X_{1}+0.03 .
\end{aligned}
$$

The plate is left oscillating at time $t=0$. Figure 14 shows the velocity distribution at two different instants of time when using $2 \times 50 \times 12$ linear triangular elements and $\alpha_{C F L}=0.4$. The solutions compare very well against those provided in [1, 92].

The dissipation of the solution is analysed in Figure 15. The evolution of the kinetic, internal and (numerical) total energies are plotted against the conserved total energy measured from the first law of thermodynamics (1d). For isothermal processes, the difference between the numerical total energy and the conserved total energy is the actual dissipation of the algorithm. It can be seen that the numerical dissipation is clearly reduced as the mesh is refined. The frequency and amplitude of the oscillations are in good agreement with the results reported in $[1,48]$. Comparison of the numerical total energy obtained using two CFD-like methodologies, namely JST and upwind FVM, is shown in Figure 16. Observe that the upwind FVM is less dissipative and thus provides a better resolution than the JST method. 


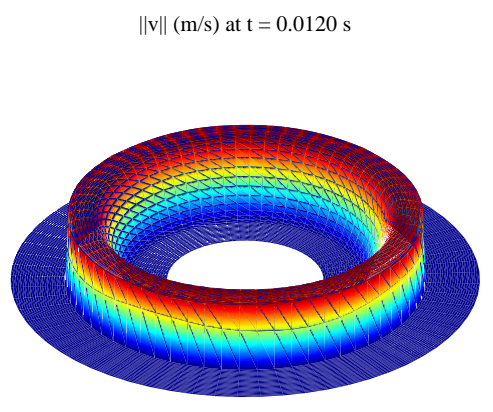

(a)

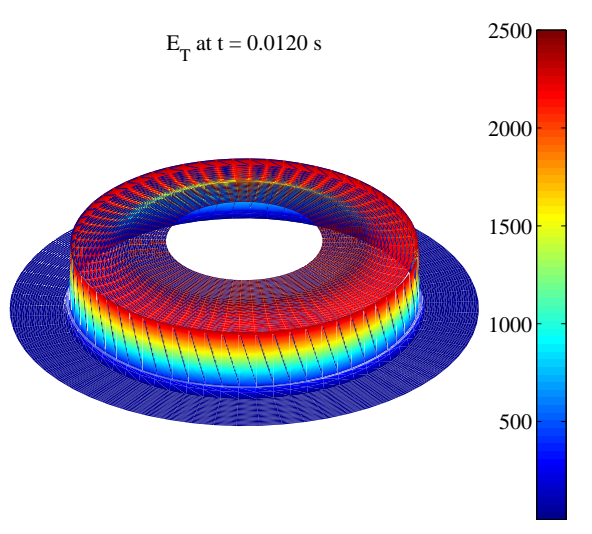

(c)
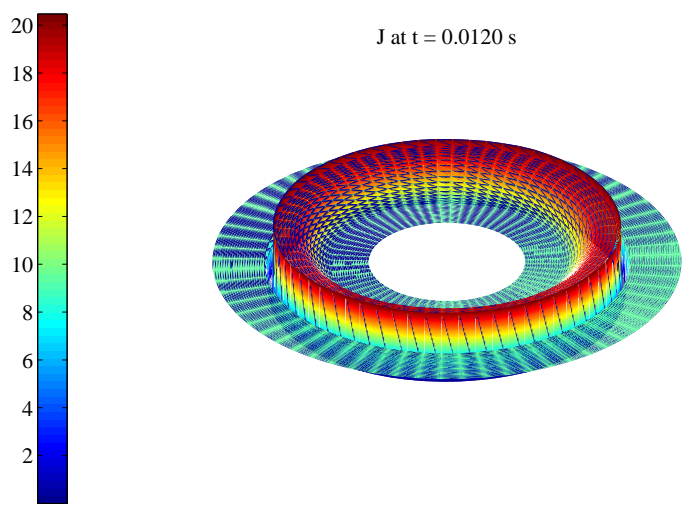

(b)

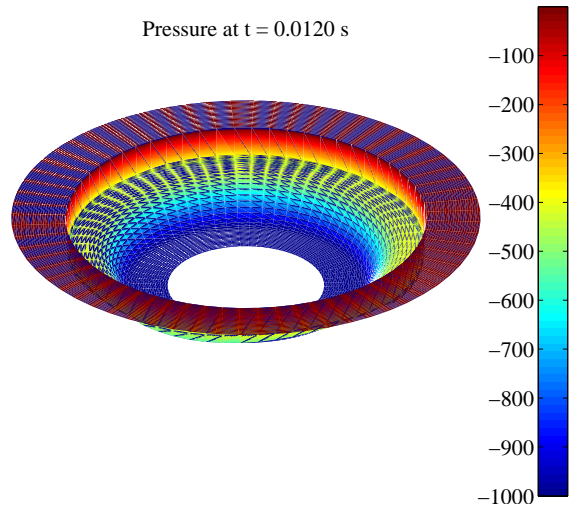

(d)

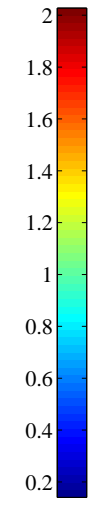

Figure 11: Axisymmetric Woodward-Colella blast test. Elevation plots for the velocity, Jacobian of the deformation, total energy and pressure at time $\mathrm{t}=0.012 \mathrm{~s}$. Ideal gas with $\Gamma_{0}=\frac{2}{5}$. Spatial discretisation using the upwind FVM with $2 \times 64 \times 64$ linear triangular elements and Barth-Jespersen limiter. Temporal discretisation using $\alpha_{C F L}=0.4$. 

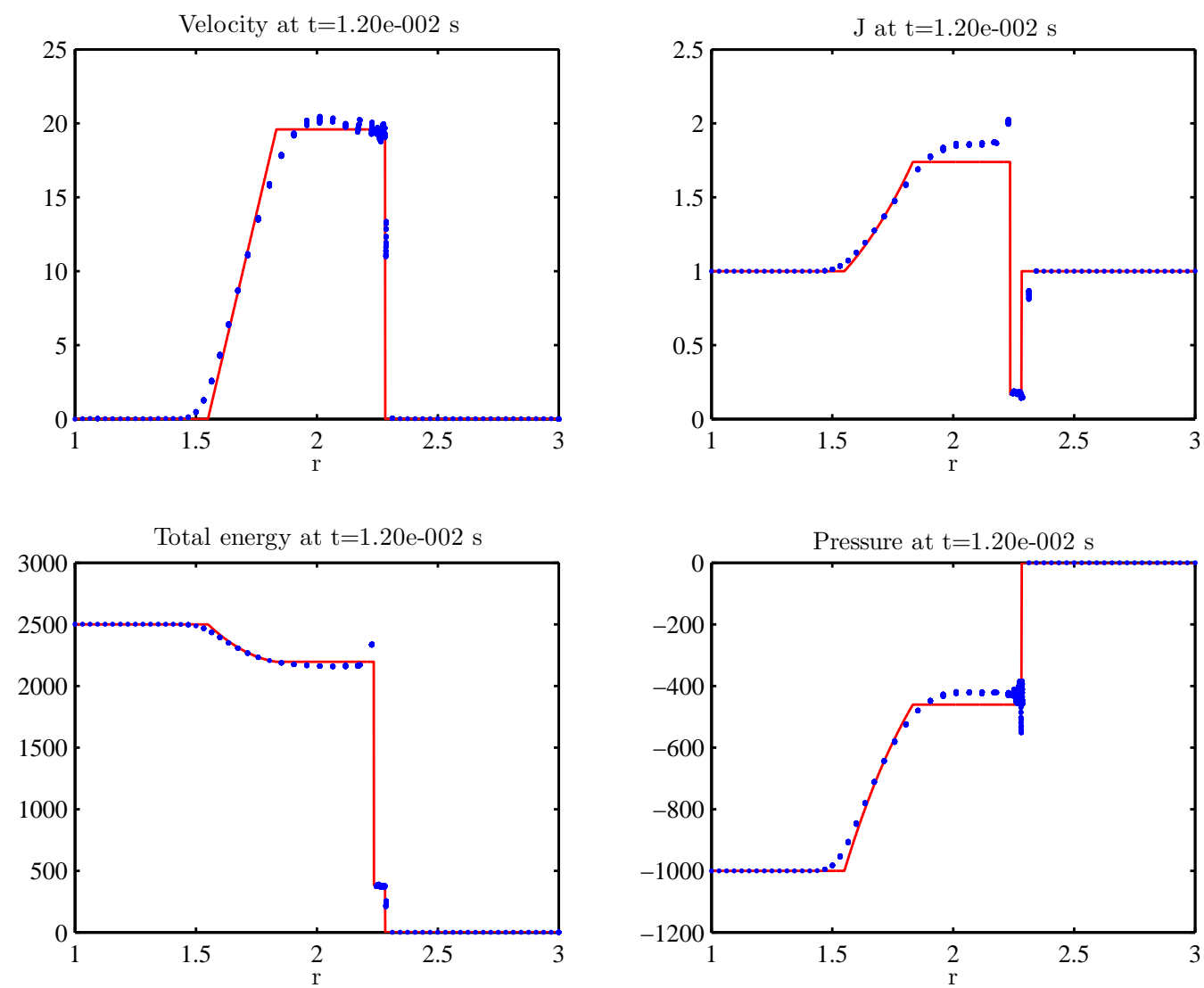

Figure 12: Axisymmetric Woodward-Colella blast test. Radial profiles for the velocity, Jacobian of the deformation, total energy and pressure at time $\mathrm{t}=0.012 \mathrm{~s}$. Ideal gas with $\Gamma_{0}=\frac{2}{5}$. Spatial discretisation using the upwind with $2 \times 64 \times 64$ linear triangular elements and Barth-Jespersen limiter. Temporal discretisation using $\alpha_{C F L}=0.4$. 


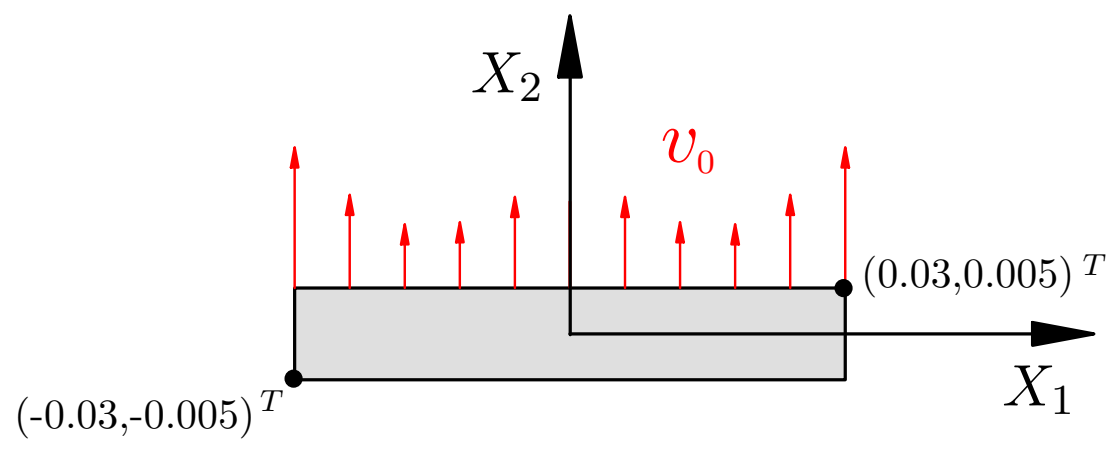

Figure 13: Beryllium plate initial configuration.

\subsection{Collapse of a thick-walled cylindrical Beryllium shell}

This test is designed to assess the ability of the algorithm in the modelling of plastic flows. It was initially proposed in [52] and later implemented in $[1,2,53]$. A Beryllium shell, of an inner radius $R_{i}=80 \cdot 10^{-3} \mathrm{~m}$ and an outer radius $R_{o}=100 \cdot 10^{-3} \mathrm{~m}$, has an initial radial velocity field. The shell collapses towards its centre until all the kinetic energy is transformed into its plastic dissipation. That instant is called the stopping time. Accordingly, the inner and outer radii at the stopping time are called the stopping radii.

In this particular case, the material is modelled using a hyperelasticplastic constitutive model (see [68] or algorithm 6.1 in [1]) together with the use of a Mie-Gruneisen shock-related equation of state (40). Its material parameters are defined as follows: density $\rho_{0}=1845 \mathrm{~kg} / \mathrm{m}^{3}$, Grüneisen coefficient $\Gamma_{0}=2, c_{p, 0}=12870 \mathrm{~m} / \mathrm{s}, s=1.124$, shear modulus $\mu=151.9 \times 10^{9}$ $\mathrm{Pa}$, yield strength $Y^{0}=330 \times 10^{6} \mathrm{~Pa}$ and hardening modulus $H=0 \mathrm{~Pa}$ (perfectly plastic material).

The initial velocity field is given by

$$
\boldsymbol{v}\left(\boldsymbol{X}, t_{0}\right)=-V_{0} \frac{R_{i}}{\left\|X_{1}^{2}+X_{2}^{2}\right\|^{2}}\left(X_{1}, X_{2}\right)^{T} \mathrm{~m} / \mathrm{s}
$$

and the exterior pressure is defined as $p=1 \times 10^{-6} \mathrm{~Pa}$. The shell is simulated using symmetric boundary conditions or roller supports (see Figure 17).

For completeness, this problem is simulated using three different initial velocities (e.g. $417.1 \mathrm{~m} / \mathrm{s}, 454.7 \mathrm{~m} / \mathrm{s}$ and $490.2 \mathrm{~m} / \mathrm{s}$ ) with various level of 


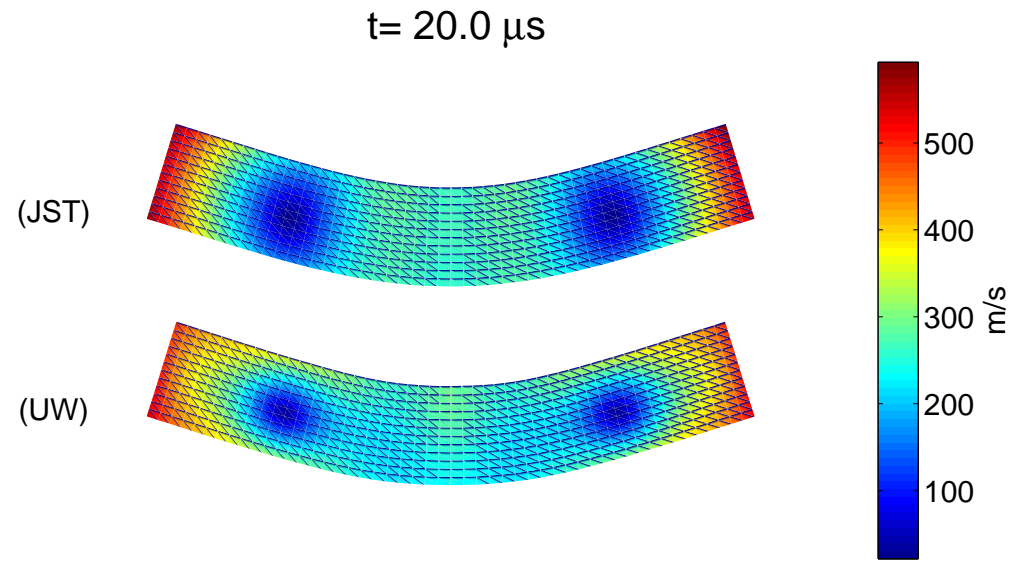

(a)

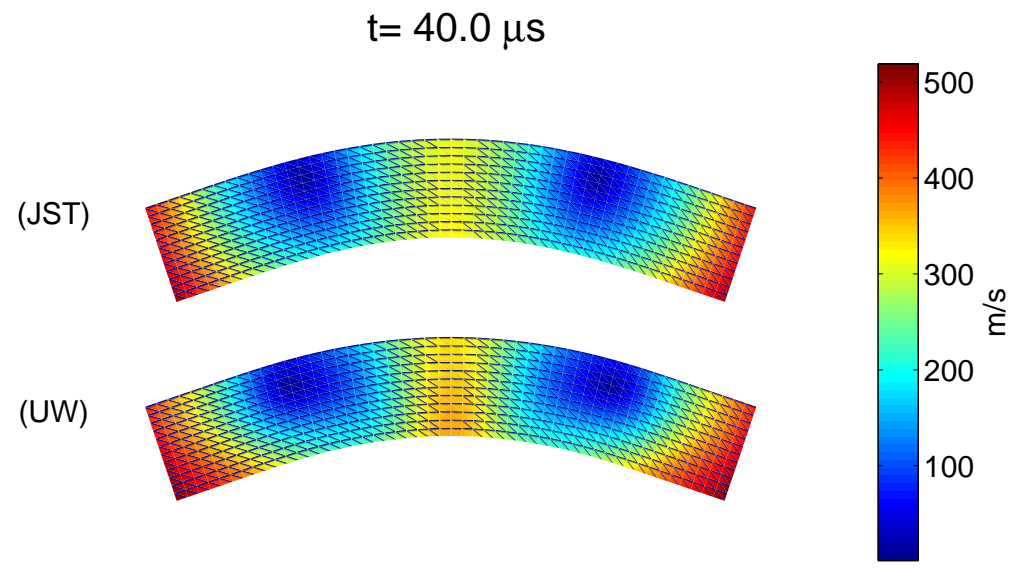

(b)

Figure 14: Beryllium plate. Material properties $\rho_{0}=1845 \mathrm{~kg} / \mathrm{m}^{3}, E=$ $3.1827 \times 10^{11} \mathrm{~Pa}, \nu=0.05390$ and yield strength $Y^{0}=1 \times 10^{11} \mathrm{~Pa}$. Evolution in time of the deformed shaped. The contour plot represents the norm of the velocity vector. Solutions obtained using $2 \times 50 \times 12$ linear triangular elements and $\alpha_{C F L}=0.4$. 


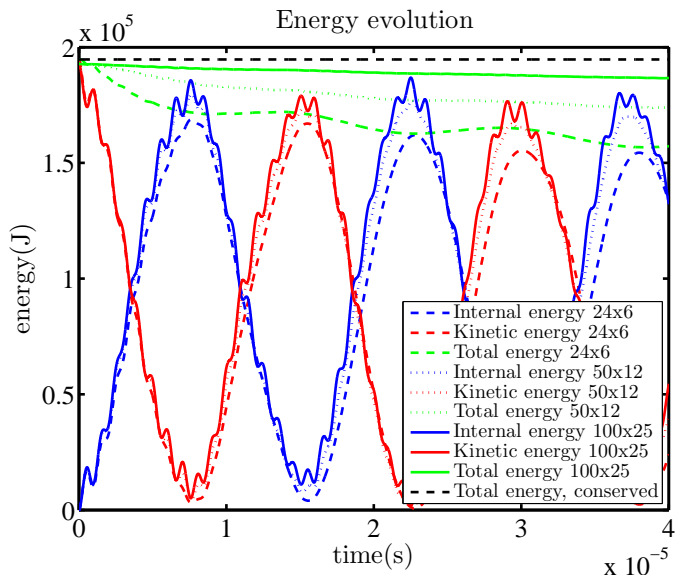

Figure 15: Beryllium plate. Material properties $\rho_{0}=1845 \mathrm{~kg} / \mathrm{m}^{3}, E=$ $3.1827 \times 10^{11} \mathrm{~Pa}, \nu=0.05390$ and yield strength $Y^{0}=1 \times 10^{11} \mathrm{~Pa}$. Evolution in time of the internal energy (blue lines), kinetic energy (red lines), summation of both (green lines) against the total conserved energy (black discontinuous line) for three different meshes of $2 \times 24 \times 6,2 \times 50 \times 12$ and $2 \times 100 \times 25$ linear triangular elements. Temporal discretisation using $\alpha_{C F L}=0.4$.

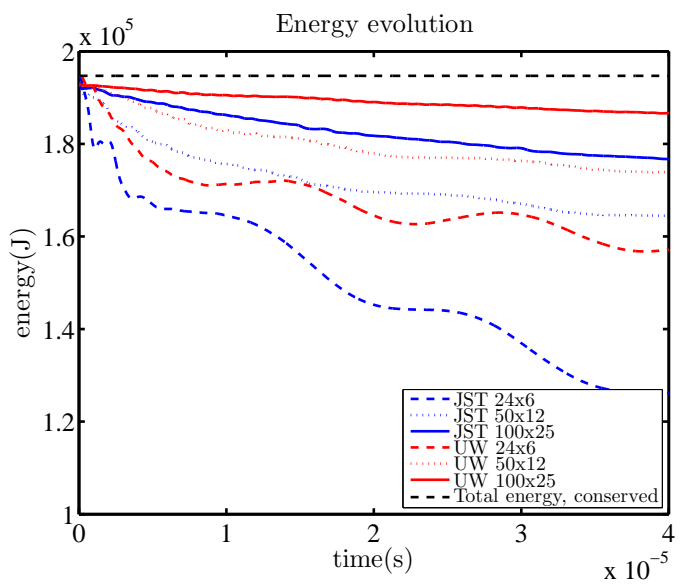

Figure 16: Beryllium plate. Material properties $\rho_{0}=1845 \mathrm{~kg} / \mathrm{m}^{3}, E=$ $3.1827 \times 10^{11} \mathrm{~Pa}, \nu=0.05390$ and yield strength $Y^{0}=1 \times 10^{11} \mathrm{~Pa}$. Comparison of the total numerical energy using both the JST and upwind FVM and for three different meshes of $2 \times 24 \times 6,2 \times 50 \times 12$ and $2 \times 100 \times 25$ linear triangular elements. 


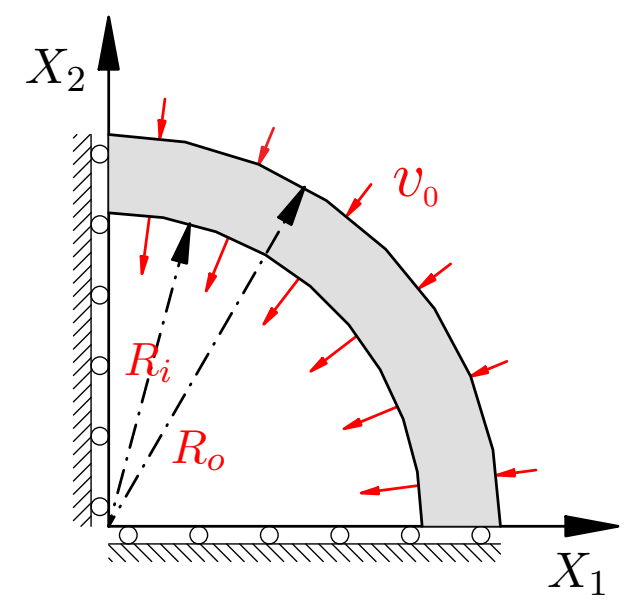

Figure 17: Beryllium shell initial configuration.

meshes $($ e.g. $2 \times(8 \times 10), 2 \times(16 \times 20)$ and $2 \times(32 \times 40)$ linear triangular elements per quarter of shell), and compared against the analytical solution provided in [52]. The time step is controlled by $\alpha_{C F L}=0.4$. The final inner and outer radii and the stopping time are shown in Table 5 . It can be clearly seen that the numerical results obtained from upwind FVM converge to the closed form solution as the mesh is refined.

It is worth mentioning that the JST solution [1] converged relatively faster than its counterpart upwind FVM. This is due to the fact that the amount of dissipation added into the JST algorithm can be tuned through the tailormade artificial dissipative parameter (see (35) in [1]). The upwind FVM, on the other hand, has a fixed physically based numerical dissipation contributed from Riemann solver, making it less convenient for problems where physical diffusion is present.

In addition, the axisymmetry of the solutions is quantified as in [52]. Table 6 shows the results associated to the standard, minimum and maximum deviations of the radius for each of the layers of the mesh. It can be seen how the algorithm is capable of preserving an excellent axisymmetry, giving maximum standard deviation of the order $10^{-9}$.

\subsection{L-shaped block}

This example was proposed by Simo et al. in [93] and later used by other authors [94, 95]. Its objective is to assess the capability of the upwind $\boldsymbol{p}$ - $\boldsymbol{F}$ - $J$ 
Table 5: Numerical results for the Beryllium shell problem. The table shows the numerical inner and outer radii, the analytical inner and outer radii and the stopping time for each of the problems and mesh discretisations $\left(n_{l}\right.$ : number of layers, $n_{s}$ : number of sectors).

\begin{tabular}{ccccccc}
\hline$V_{0}$ & $n_{l} \times n_{s}$ & $R_{i}^{\text {num }}(\mathrm{mm})$ & $R_{i}^{\text {an }}(\mathrm{mm})$ & $R_{o}^{\text {num }}(\mathrm{mm})$ & $R_{o}^{\text {an }}(\mathrm{mm})$ & $t_{f}(\mu \mathrm{s})$ \\
\hline 417.1 & $8 \times 10$ & 52.47 & 50.00 & 79.65 & 78.10 & 117.07 \\
417.1 & $16 \times 20$ & 50.38 & 50.00 & 78.32 & 78.10 & 124.27 \\
417.1 & $32 \times 40$ & 50.06 & 50.00 & 78.12 & 78.10 & 125.35 \\
454.7 & $8 \times 10$ & 48.36 & 45.00 & 76.99 & 75.00 & 120.72 \\
454.7 & $16 \times 20$ & 45.53 & 45.00 & 75.29 & 75.00 & 129.64 \\
454.7 & $32 \times 40$ & 45.08 & 45.00 & 75.03 & 75.00 & 131.06 \\
490.2 & $8 \times 10$ & 44.89 & 40.00 & 74.61 & 72.12 & 122.67 \\
490.2 & $16 \times 20$ & 40.08 & 40.00 & 72.50 & 72.12 & 133.43 \\
490.2 & $32 \times 40$ & 40.04 & 40.00 & 72.17 & 72.12 & 135.32 \\
\hline
\end{tabular}

Table 6: Numerical results for the Beryllium shell problem. The table shows the standard deviation of the radius for each of the layers of the mesh. The minimum and maximum deviation among all the nodes is as well presented $\left(n_{l}\right.$ : number of layers, $n_{s}$ : number of sectors).

\begin{tabular}{ccccc}
\hline$V_{0}$ & $n_{l} \times n_{s}$ & $\begin{array}{c}\text { Standard } \\
\text { deviation } \sigma(\%)\end{array}$ & $\begin{array}{c}\text { Minimum } \\
\text { deviation }(\%)\end{array}$ & $\begin{array}{c}\text { Maximum } \\
\text { deviation }(\%)\end{array}$ \\
\hline \hline 417.1 & $8 \times 10$ & $3.08 \times 10^{-10}$ & $-9.43 \times 10^{-11}$ & $1.29 \times 10^{-11}$ \\
417.1 & $16 \times 20$ & $3.27 \times 10^{-10}$ & $-9.48 \times 10^{-12}$ & $1.62 \times 10^{-11}$ \\
417.1 & $32 \times 40$ & $4.74 \times 10^{-10}$ & $-2.71 \times 10^{-11}$ & $2.14 \times 10^{-11}$ \\
454.7 & $8 \times 10$ & $2.71 \times 10^{-10}$ & $-7.39 \times 10^{-12}$ & $9.15 \times 10^{-12}$ \\
454.7 & $16 \times 20$ & $5.31 \times 10^{-10}$ & $-1.96 \times 10^{-11}$ & $2.23 \times 10^{-11}$ \\
454.7 & $32 \times 40$ & $6.24 \times 10^{-10}$ & $-3.62 \times 10^{-11}$ & $3.15 \times 10^{-11}$ \\
490.2 & $8 \times 10$ & $4.46 \times 10^{-10}$ & $-1.32 \times 10^{-11}$ & $1.41 \times 10^{-11}$ \\
490.2 & $16 \times 20$ & $7.56 \times 10^{-10}$ & $-4.63 \times 10^{-11}$ & $5.05 \times 10^{-11}$ \\
490.2 & $32 \times 40$ & $1.10 \times 10^{-9}$ & $-5.95 \times 10^{-11}$ & $6.42 \times 10^{-11}$ \\
\hline
\end{tabular}




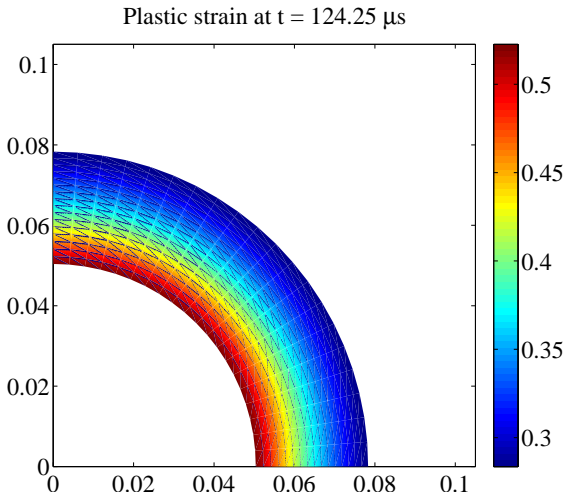

(a)

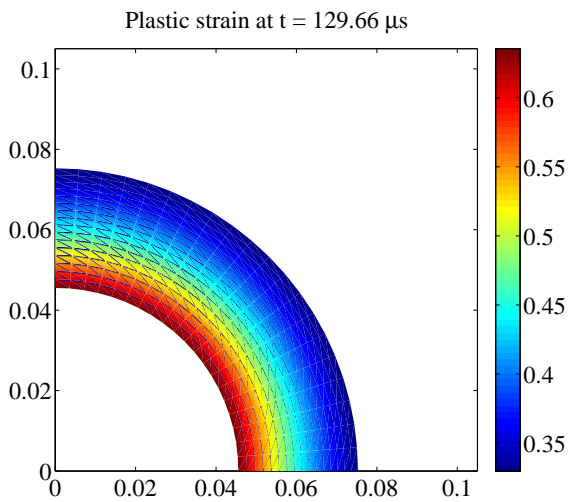

(c)

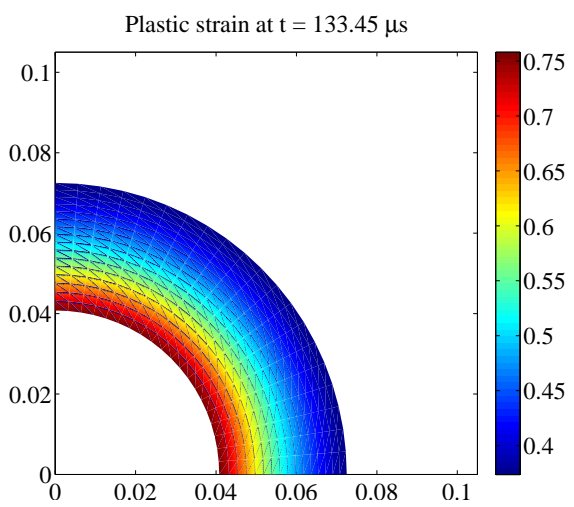

(e)

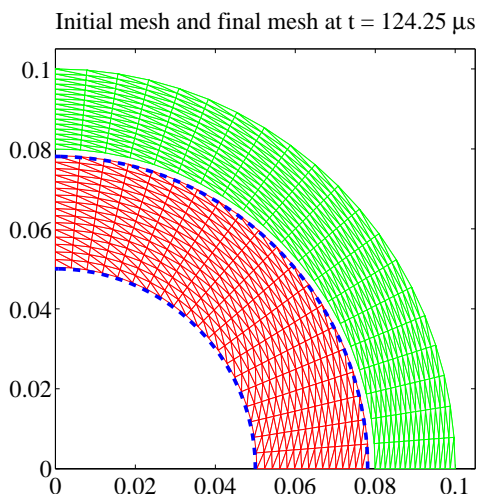

(b)

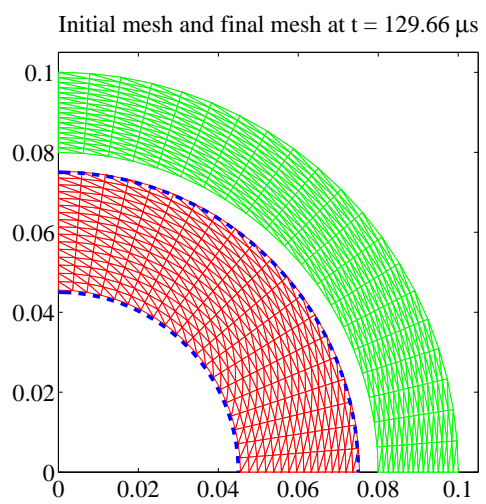

(d)

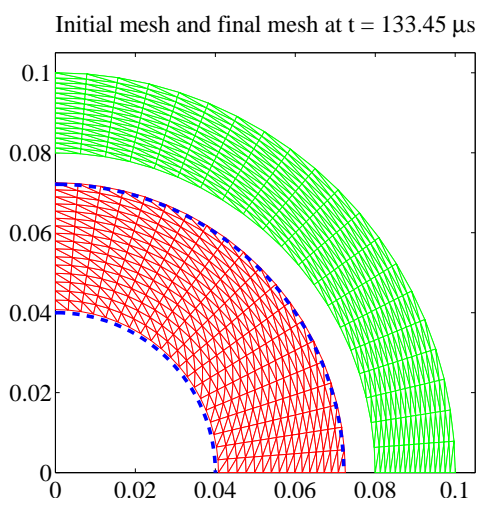

(f)

Figure 18: Beryllium shell problem. Hyperelastic-plastic constitutive model and Mie-Gruneisen equation of state. Material parameters: $\rho_{0}=1845 \mathrm{~kg} / \mathrm{m}^{3}$, $\Gamma_{0}=2, c_{0}=12870 \mathrm{~m} / \mathrm{s}, s=1.124, \mu=151.9 \times 10^{9} \mathrm{~Pa}, Y^{0}=330 \times 10^{6}$ $\mathrm{Pa}$ and $H=0 \mathrm{~Pa}$. Mesh of $2 \times 405_{0} 32$ linear triangular elements. From top to bottom rows, results are shown for initial velocities $\boldsymbol{v}_{0}=417.1 \mathrm{~m} / \mathrm{s}$, $\boldsymbol{v}_{0}=454.7 \mathrm{~m} / \mathrm{s}$ and $\boldsymbol{v}_{0}=490.2 \mathrm{~m} / \mathrm{s}$ at their stopping time. Plastic strain is shown in the left column. Initial mesh (green) and final mesh (red) against the analytical solution (thick blue line) are shown in the right column. 
mixed FVM in preserving the angular momentum within a system. A threedimensional L-shaped block is subjected to time varying forces at both of its sides (see Figure 19) and is imposed traction-free condition at the rest of the boundaries. The forces are described by the following equations

$$
\boldsymbol{F}_{1}(t)=-\boldsymbol{F}_{2}(t)=(150,300,450) p(t), \quad p(t)= \begin{cases}t, & 0 \leq t<2.5 \\ 5-t, & 2.5 \leq t<5 \\ 0, & t \geq 5\end{cases}
$$

In this particular case, a Neo-Hookean model is chosen and its material properties are: $\mu=1.925 \times 10^{4} \mathrm{~Pa}, \lambda=2.885 \times 10^{4} \mathrm{~Pa}$ and $\rho_{0}=1.0 \times 10^{3} \mathrm{~kg} / \mathrm{m}^{3}$. The simulation is run using 388 elements for the spatial discretisation and $\alpha_{C F L}=0.4$. Figure 20 shows a series of snapshots of the deformed configuration at different times. Figure 21 demonstrates the ability of the algorithm to preserve the angular momentum (once the external forces are released) and linear momentum (the external torque is applied at the centre of mass of the block).

It is important to note that the correction in the internal tractions is very small, as compared to the actual value of these tractions. This is shown in Figures 22 and 23. Figure 22 shows the distribution of the ratio between the norm of the modification of the edge forces against the norm of the actual edge forces, that is $\frac{\left\|\hat{\boldsymbol{f}}_{k}-\boldsymbol{f}_{k}\right\|}{\left\|\boldsymbol{f}_{k}\right\|}$. Figure 23 shows the time history of the $L^{2}$ norm of the edge forces, $\boldsymbol{f}_{k}$, against the $L^{2}$ norm of the modification of the edge forces $\hat{\boldsymbol{f}}_{k}-\boldsymbol{f}_{k}$.

Finally, in order to prove the convergence of the method, the problem is run with a finer mesh of 1178 nodes. Figure 24 shows the deformed shape for different time instants, where clearly more detailed pressure distribution patterns can be observed. Figure 25 compares the numerical total energy (summation of the linear momentum and internal energy) against the preserved total energy (obtained from solving equation (1d)) for the two different meshes. It can be seen that, as the mesh is refined, the dissipation of the solution is clearly reduced.

\subsection{Bending column}

The following example assesses the behaviour of the numerical method in bending dominated scenarios $[1,5]$. A rubber-like material column with a cross section of $1 \times 1 \mathrm{~m}^{2}$ and $6 \mathrm{~m}$ tall (see Figure 26) is clamped on its 


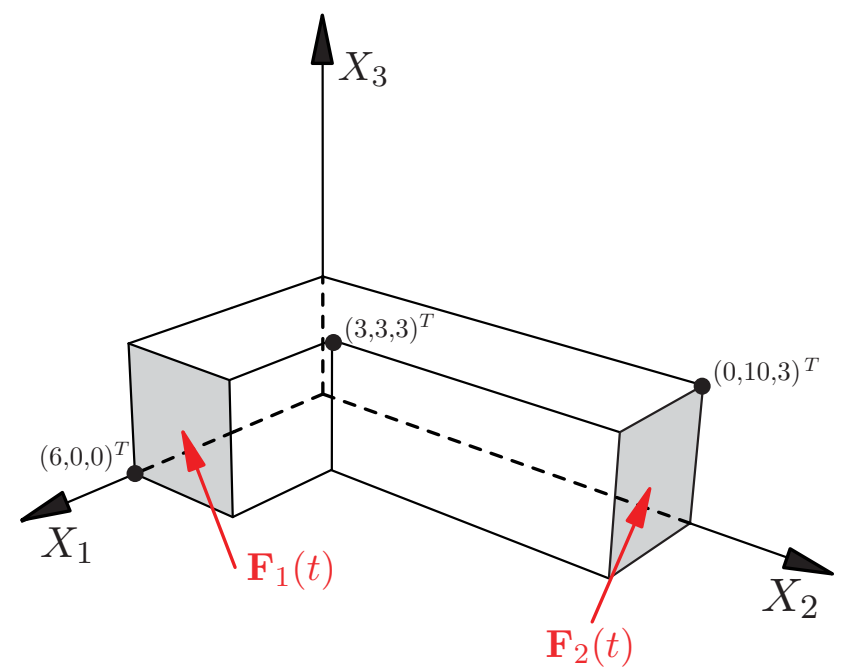

Figure 19: L-shaped block.

bottom face $\left(X_{3}=0\right)$ and has an initial uniform velocity given by

$$
\boldsymbol{v}(\boldsymbol{X}, t=0)=10\left(\frac{\sqrt{3}}{2}, \frac{1}{2}, 0\right) \mathrm{m} / \mathrm{s} \quad \text { if } X_{3}>0 .
$$

The bar is left oscillating freely in time and a Neo-Hookean material is chosen such that Young's modulus $E=1.7 \times 10^{7} \mathrm{~Pa}$ and density $\rho_{0}=1.1 \times 10^{3}$ $\mathrm{kg} / \mathrm{m}^{3}$. To assess the ability of the algorithm in near incompressibility limit, a range of Poisson's ratios will be examined.

Figure 27 shows the evolution in time of the deformed shape for a mesh discretisation of $2 \times(2 \times 2 \times 12)$ elements, $\alpha_{C F L}=0.4$ and a Poisson's ratio $\nu=0.495$. Figure 27 a shows the solution simulated using the upwind $\boldsymbol{p}-\boldsymbol{F}$ FVM, in which non-physical hydrostatic pressure modes are clearly seen. As it is proven in [5], this can be overcome by adding the volume map conservation law (65c) into the existing two-field $\boldsymbol{p}$ - $\boldsymbol{F}$ formulation. The flexilibity associated with the volumetric deformation is further enhanced by adding extra velocity stabilisation (65c). This will alleviate the hydrostatic pressure oscillations typically appeared in nearly incompressible deformations. However, this was not the case with $\boldsymbol{p}-\boldsymbol{F}$ formulation, since no velocity correction (see equation (66)) can be added with the aims at satisfying the involutions. For this reason, a sequence of locking-free deformed states modelled 

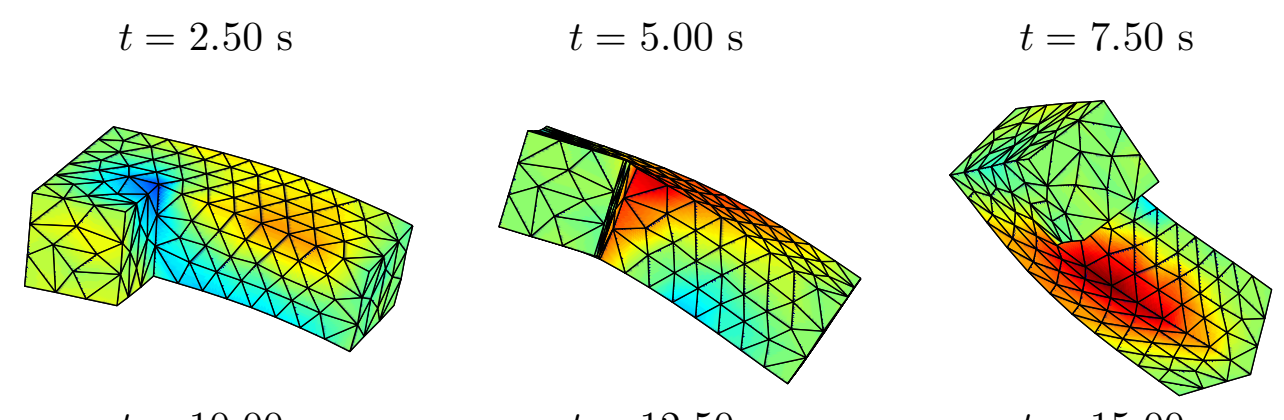

$t=10.00 \mathrm{~s}$

$t=12.50 \mathrm{~s}$

$$
t=15.00 \mathrm{~s}
$$
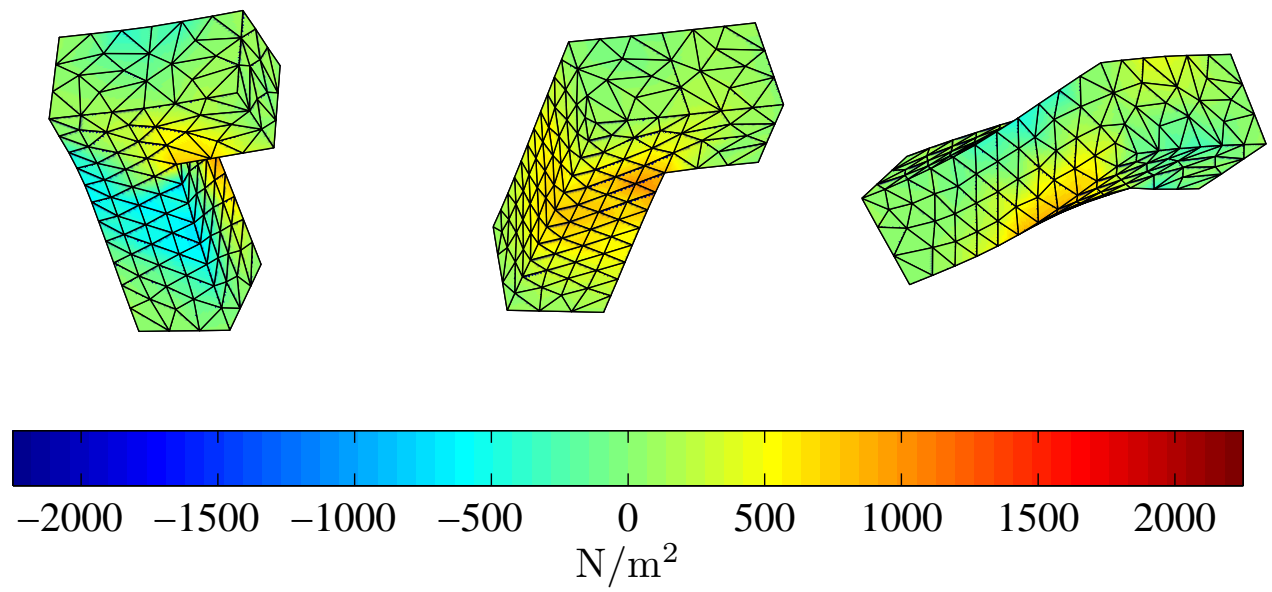

Figure 20: L-shaped block. Evolution in time of deformation and pressure distribution. Neo-Hookean material with material properties $\mu=1.925 \times$ $10^{4} \mathrm{~Pa}, \lambda=2.885 \times 10^{4} \mathrm{~Pa}$ and $\rho_{0}=1.0 \times 10^{3} \mathrm{~kg} / \mathrm{m}^{3}$. Upwind spatial discretisation using a linear tetrahedral mesh of 388 nodes and $\alpha_{C F L}=0.4$. 


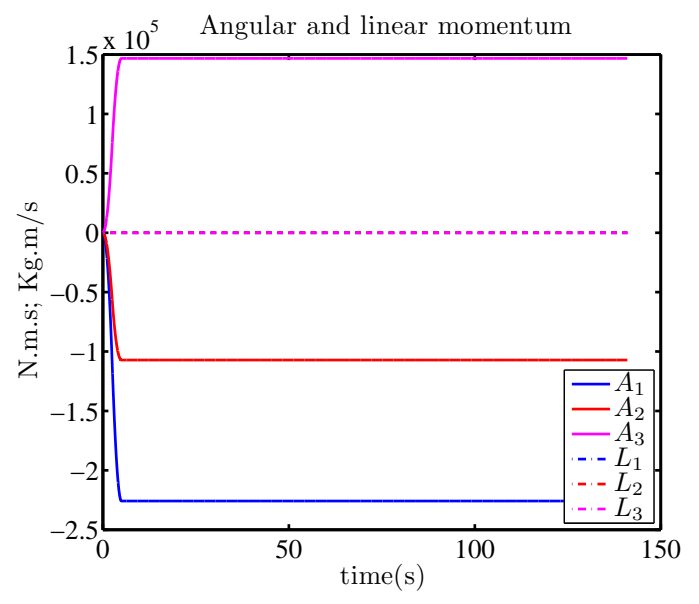

Figure 21: L-shaped block. Neo-Hookean material with material properties $\mu=1.925 \times 10^{4} \mathrm{~Pa}, \lambda=2.885 \times 10^{4} \mathrm{~Pa}$ and $\rho_{0}=1.0 \times 10^{3} \mathrm{~kg} / \mathrm{m}^{3}$. Upwind $\mathrm{FVM}$ and $\alpha_{C F L}=0.4$. Conservation of linear momentum $\boldsymbol{p}=\left[L_{1}, L_{2}, L_{3}\right]^{T}$ and angular momentum $\boldsymbol{A}=\left[A_{1}, A_{2}, A_{3}\right]^{T}$ for a mesh of 388 nodes.

using the enhanced $\boldsymbol{p}$ - $\boldsymbol{F}-J$ upwind FVM is depicted in Figure 27b, showing smooth pressure distribution without spurious modes. Figure 28 shows the robustness of the $\boldsymbol{p}-\boldsymbol{F}-J$ upwind FVM formulation by comparing the solution at time $t=2.45 \mathrm{~s}$ (i.e. two and a half cycles) with three different values of Poisson's ratios: $\nu=0.45, \nu=0.495$ and $\nu=0.4995$ and the same spatial and temporal discretisations as in the previous example. It is worth pointing out that these solutions are obtained with only two elements across the thickness, without exhibiting detrimental locking difficulties. This would not be possible with either the JST discretisation [1] or any classical displacement based formulations in bending dominated nearly incompressible deformations.

\subsection{Twisting column}

This example examines the robustness of the proposed $\boldsymbol{p}-\boldsymbol{F}-J$ upwind FVM when dealing with problems involving highly nonlinear large deformations [5]. A squared section column of $1 \mathrm{~m}$ side length and $6 \mathrm{~m}$ height is clamped on its bottom and subjected to an initial rotational velocity field given by

$$
\boldsymbol{v}(\boldsymbol{X}, t=0)=\boldsymbol{\omega}_{0} \times \boldsymbol{X} ; \quad \boldsymbol{\omega}_{0}=\left[0,0, \Omega \sin \left(\frac{\pi X_{3}}{2 L}\right)\right]^{T}
$$



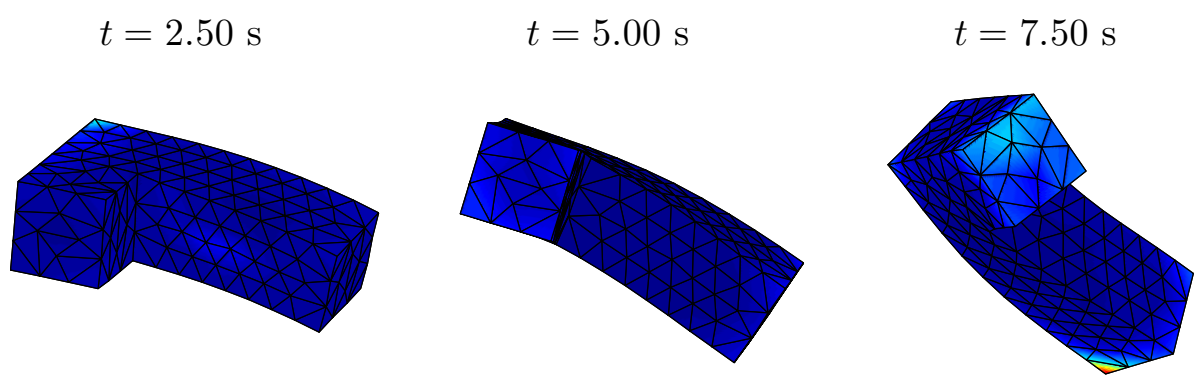

$$
t=10.00 \mathrm{~s}
$$

$t=12.50 \mathrm{~s}$

$$
t=15.00 \mathrm{~s}
$$
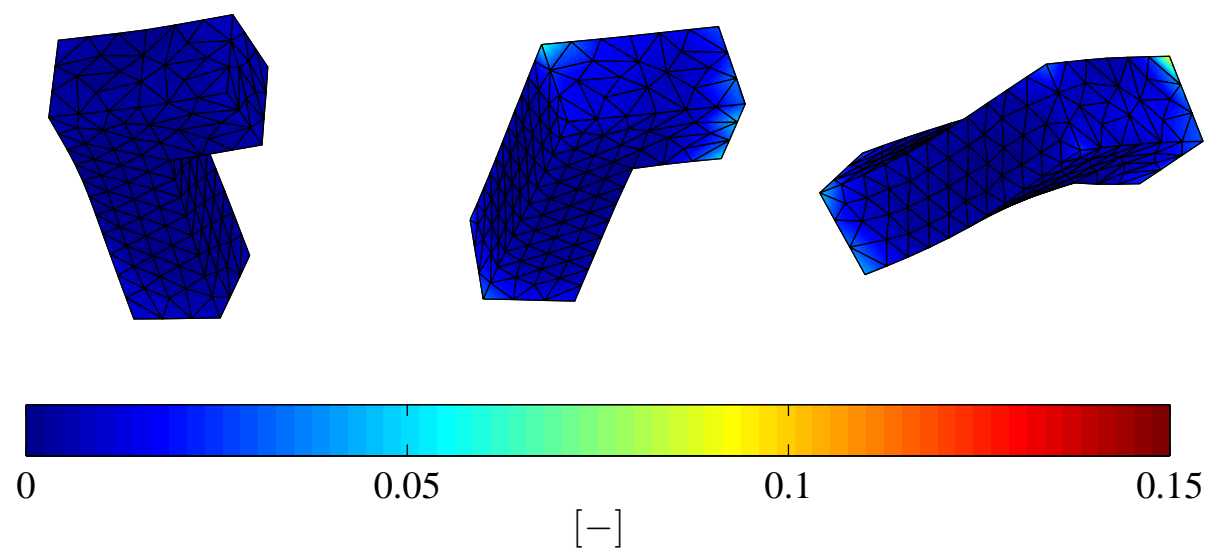

Figure 22: L-shaped block, distribution of the normalised norm of the vector resulting from the difference between the modified edge forces, and the original edge forces, $\frac{\left\|\left(\boldsymbol{f}_{k}-\hat{\boldsymbol{f}}_{k}\right)\right\|}{\left\|\boldsymbol{f}_{k}\right\|}$. The plot shows the average at the nodes from its surrounding edge values. Neo-Hookean material with material properties $\mu=1.925 \times 10^{4} \mathrm{~Pa}, \lambda=2.885 \times 10^{4} \mathrm{~Pa}, \rho_{0}=1.0 \times 10^{3} \mathrm{~kg} / \mathrm{m}^{3}$. Upwind spatial discretisation using a linear tetrahedral mesh of 388 nodes and $\alpha_{C F L}=0.4$. 

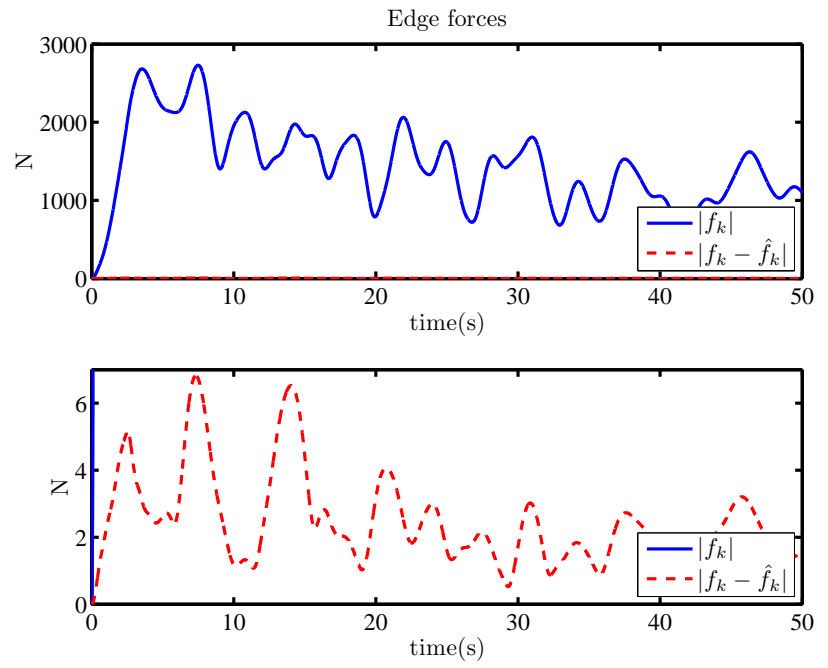

Figure 23: L-shaped block, evolution of the $L^{2}$ norm of the edge forces (blue) against the $L^{2}$ norm of the modification of the edge forces (red). The bottom plot shows a zoomed version of the above plot. Neo-Hookean material with material properties $\mu=1.925 \times 10^{4} \mathrm{~Pa}, \lambda=2.885 \times 10^{4} \mathrm{~Pa}, \rho_{0}=1.0 \times$ $10^{3} \mathrm{~kg} / \mathrm{m}^{3}$. Upwind spatial discretisation using a linear tetrahedral mesh of 388 nodes and $\alpha_{C F L}=0.4$. 

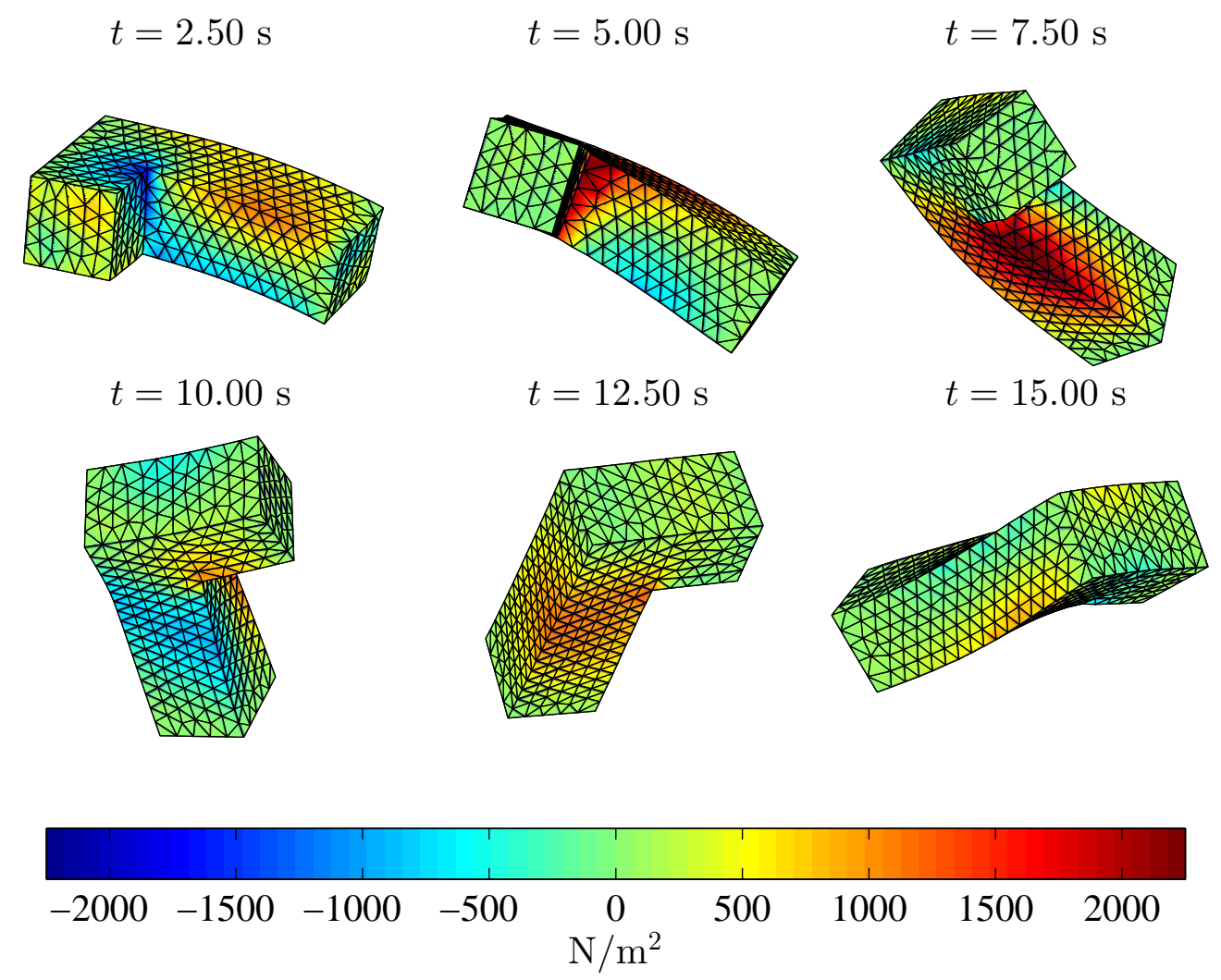

Figure 24: L-shaped block. Evolution in time of deformation and pressure distribution. Neo-Hookean material with material properties $\mu=1.925 \times$ $10^{4} \mathrm{~Pa}, \lambda=2.885 \times 10^{4} \mathrm{~Pa}$ and $\rho_{0}=1.0 \times 10^{3} \mathrm{~kg} / \mathrm{m}^{3}$. Upwind spatial discretisation using a linear tetrahedral mesh of 1178 nodes and $\alpha_{C F L}=0.4$. 


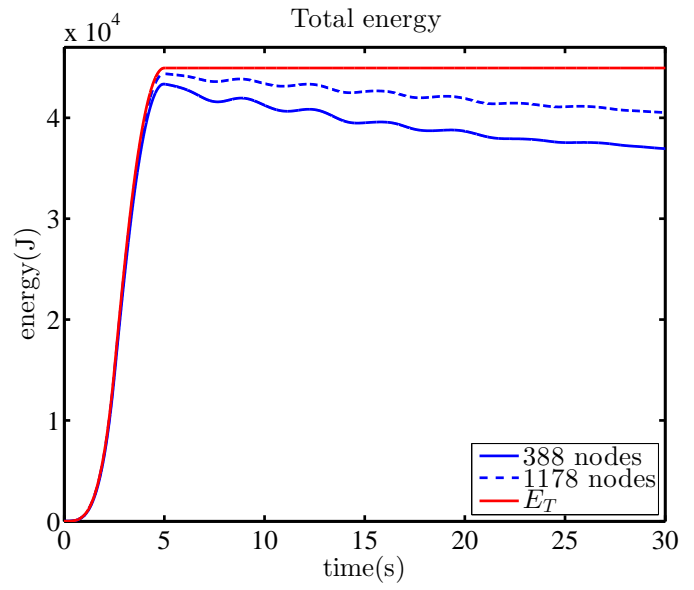

Figure 25: L-shaped block. Neo-Hookean material with material properties $\mu=1.925 \times 10^{4} \mathrm{~Pa}, \lambda=2.885 \times 10^{4} \mathrm{~Pa}$ and $\rho_{0}=1.0 \times 10^{3} \mathrm{~kg} / \mathrm{m}^{3}$. Comparison of the total numerical energy using the upwind FVM for two different meshes of 388 and 1178 nodes.

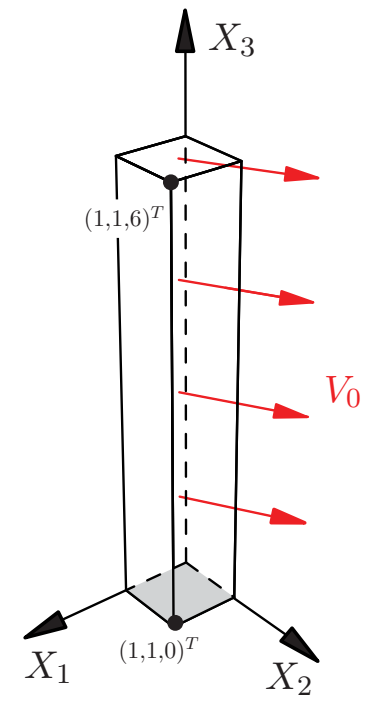

Figure 26: Three dimensional bending column. 

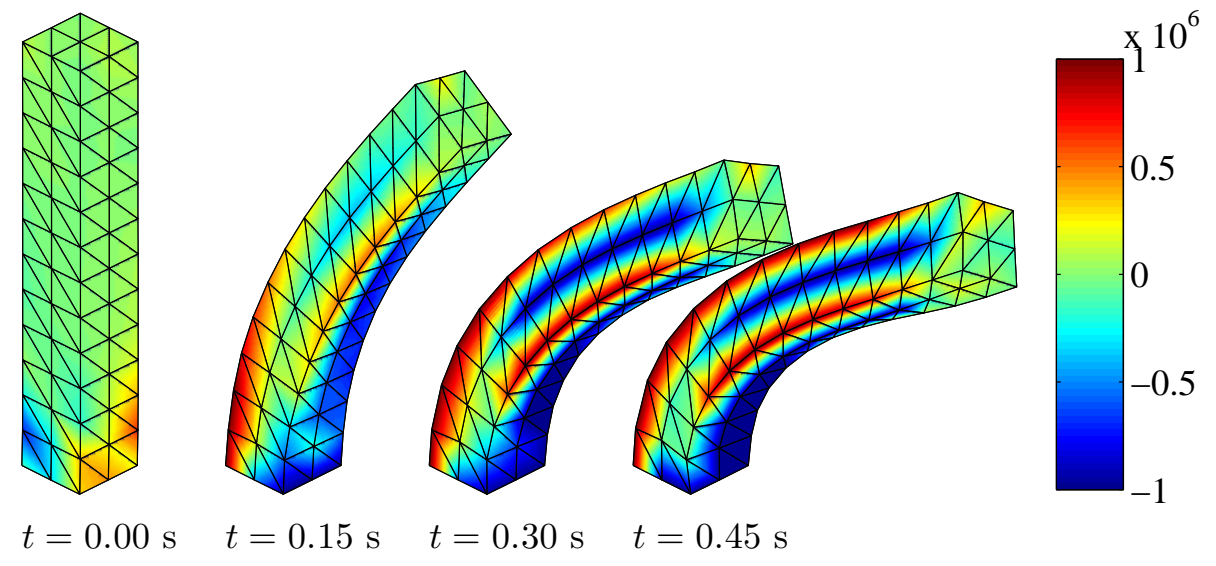

$t=0.00 \mathrm{~s}$

$t=0.15 \mathrm{~s}$

(a) Upwind $\boldsymbol{p}-\boldsymbol{F}$ formulation.
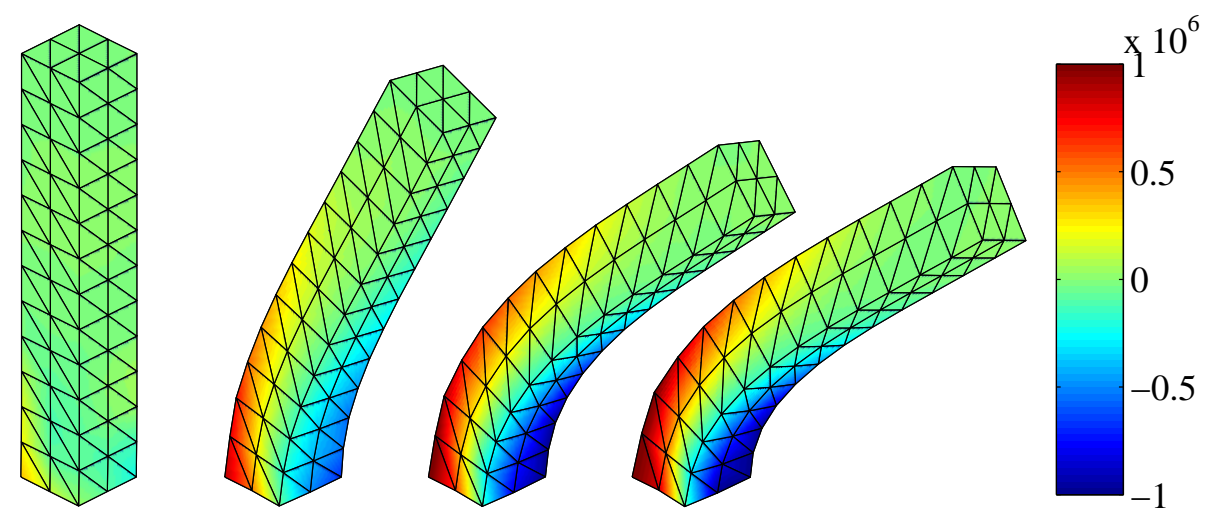

$t=0.00 \mathrm{~s}$

$t=0.15 \mathrm{~s}$

$t=0.30 \mathrm{~s} \quad t=0.45 \mathrm{~s}$

(b) Upwind $\boldsymbol{p}-\boldsymbol{F}-J$ formulation.

Figure 27: Bending column. Evolution in time of deformation and pressure distribution: (a) Upwind $\boldsymbol{p}-\boldsymbol{F}$ formulation; and (b) Upwind $\boldsymbol{p}-\boldsymbol{F}-J$ formulation. Neo-Hookean material with material properties $E=1.7 \times 10^{7} \mathrm{~Pa}$, density $\rho_{0}=1.1 \times 10^{3} \mathrm{Kg} / \mathrm{m}^{3}$ and $\nu=0.495$. Linear tetrahedral mesh of $6 \times 2 \times 2 \times 12$ and $\alpha_{C F L}=0.4$. 


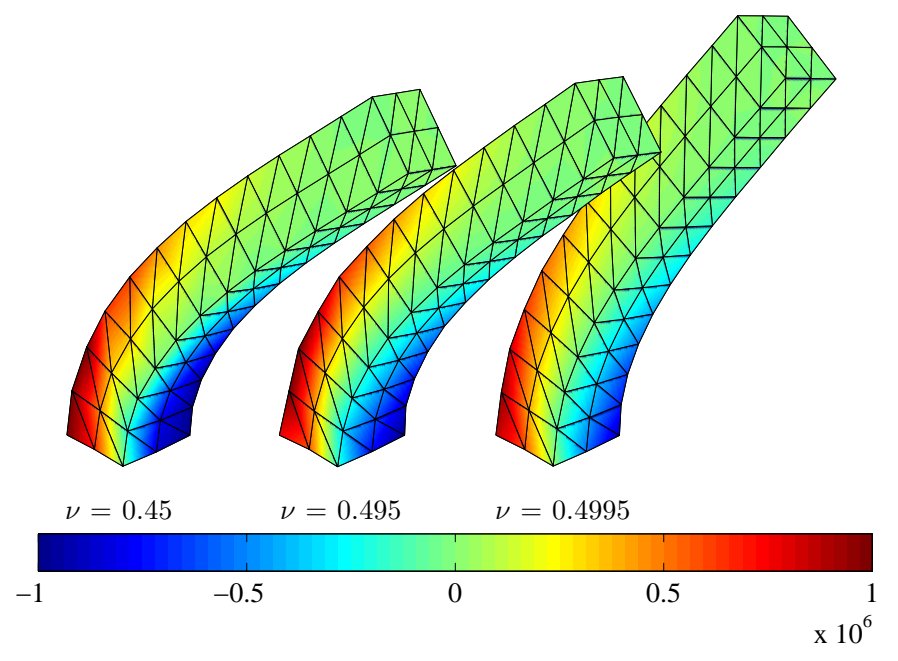

Figure 28: Bending column. Comparison of the deformed shape at time $t=$ $2.45 \mathrm{~s}$ for three different Poisson ratios. Neo-Hookean material with material properties $E=1.7 \times 10^{7} \mathrm{~Pa}$ and density $\rho_{0}=1.1 \times 10^{3} \mathrm{~kg} / \mathrm{m}^{3}$. Upwind spatial discretisation of the $\boldsymbol{p}-\boldsymbol{F}$ - $J$ formulation using a linear tetrahedral mesh of $6 \times 2 \times 2 \times 12$ and $\alpha_{C F L}=0.4$.

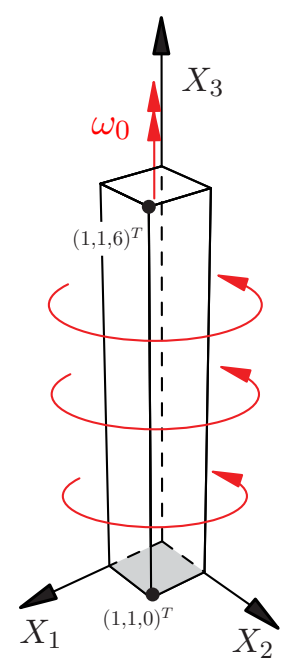

Figure 29: Setup of the column twist problem. 
This problem is modelled using a compressible Mooney-Rivlin material with parameters $\alpha=\beta=\frac{\mu}{4}$ (see equation (9)). Material properties are Young's modulus $E=1.7 \times 10^{7} \mathrm{~Pa}$, material density $\rho_{0}=1.1 \times 10^{3} \mathrm{~kg} / \mathrm{m}^{3}$ and Poisson's ratio $\nu=0.3$.

For benchmarking purposes, we simulate the exact same problem using available methodologies, namely the JST $\boldsymbol{p}$ - $\boldsymbol{F}-J$ formulation [1] and the stabilised $\boldsymbol{p}-\boldsymbol{F}-J$ Petrov-Galerkin formulation [5]. Figure 30 shows a series of snapshots using three different discretisation techniques: JST, stabilised Petrov-Galerkin and Upwind FVM. It can be clearly seen that, with the same number of meshes $(6 \times 6 \times 6 \times 36$ elements $)$, the upwind FVM solution resembles the one obtained using the Petrov-Galerkin formulation. In the case of the JST algorithm, a finer mesh of $6 \times 12 \times 12 \times 72$ linear tetrahedral elements is required in order to obtain similar results, due to the higher numerical dissipation introduced by the scheme. All examples are simulated using $\alpha_{C F L}=0.4$

\section{Conclusions}

In this paper, a new computational framework adapted from CFD has been presented for the numerical analysis of shock and nearly incompressible bending dominated scenarios for both isothermal and non-isothermal materials. The methodology is based on a system of first order conservation laws, where the linear momentum $\boldsymbol{p}$, the deformation gradient $\boldsymbol{F}$, the Jacobian of the deformation $J$ and the total energy $E$ are regarded as four primary conservation variables in this mixed approach. To complete the above system, a Mie-Grüneisen equation of state associated with volumetric deformation is presented for thermo-elastic materials. A thorough study of the eigenstructure analysis of the full system is presented ensuring the satisfaction of the rank one convexity condition.

From the spatial discretisation point of view, a second order edge-based upwind Finite Volume Method (FVM) typically used in the field of fluid dynamics is presented. In this paper, an acoustic Riemann solver derived from the associated Rankine-Hugoniot jump conditions is used. To this end, a comprehensive list of numerical examples in one, two and three dimensions has been presented. The overall methodology shows excellent behaviour in both shock-related hydrodynamics problems [55, 89, 96-98] and bending dominated nearly incompressible solids. 

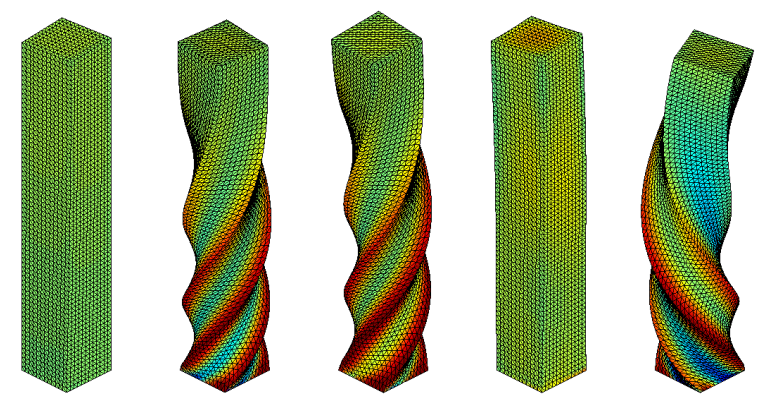

$t=0.00 \mathrm{~s}$

$t=0.06 \mathrm{~s}$

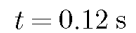

$t=0.18 \mathrm{~s}$

$t=0.24 \mathrm{~s}$

(a) JST $\boldsymbol{p}-\boldsymbol{F}-J$ formulation
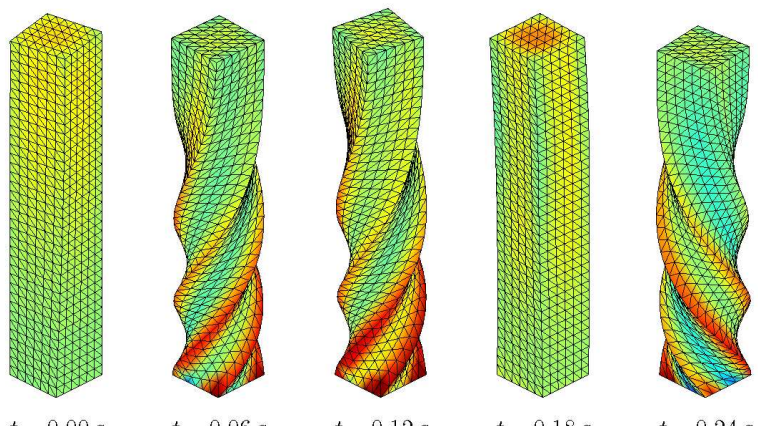

$t=0.00 \mathrm{~s}$

$t=0.06 \mathrm{~s}$

$t=0.12 \mathrm{~s}$

$t=0.18 \mathrm{~s}$

$t=0.24 \mathrm{~s}$

(b) Stabilised $\boldsymbol{p}-\boldsymbol{F}-J$ Petrov-Galerkin formulation
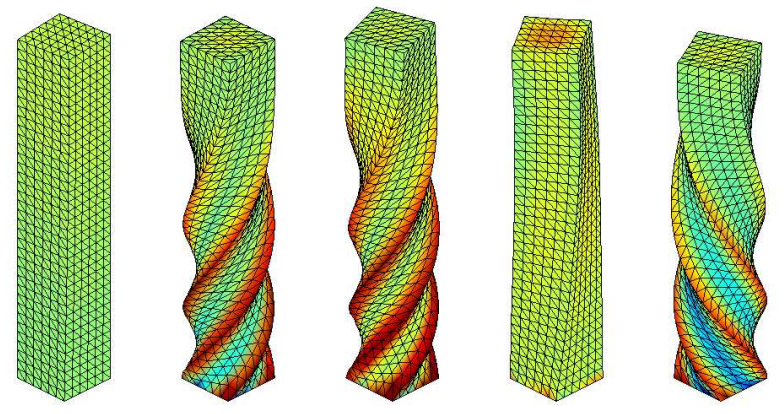

$t=0.00 \mathrm{~s}$

$t=0.06 \mathrm{~s}$

$t=0.12 \mathrm{~s}$

$t=0.18 \mathrm{~s}$

$t=0.24 \mathrm{~s}$

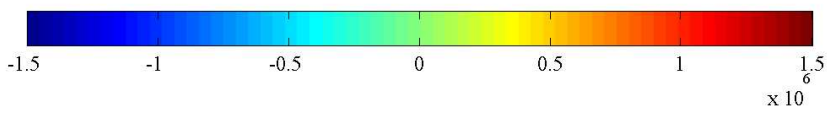

(c) Upwind $\boldsymbol{p}-\boldsymbol{F}-J$ FVM

Figure 30: Twisting column. Evolution in time of deformation and pressure distribution: (a) JST $\boldsymbol{p}$ - $\boldsymbol{F}-J$ formulation $(6 \times 12 \times 12 \times 72)$; (b) Stabilised $\boldsymbol{p}$ - $\boldsymbol{F}$ - $J$ Petrov-Galerkin formulation $(6 \times 6 \times 6 \times 36)$; and (c) Upwind $\boldsymbol{p}$ - $\boldsymbol{F}$ - $J$ FVM $(6 \times 6 \times 6 \times 36)$. Compressible Mooney-Rivlin material with parameters $\alpha=\beta=\frac{\mu}{4}, E=1.7 \times 10^{7} \mathrm{~Pa}, \rho_{0}=1.1 \times 10^{3} \mathrm{~kg} / \mathrm{m}^{3}$ and $\nu=0.3$. 
The consideration of an iterative Riemann solver [99] to further enhance the robustness of the formulation is the next step of our work along with an alternative Runge-Kutta time integrator tailor-made to preserve angular momentum without the need to employ a posteriori projection procedure.

\section{Acknowledgements}

The first author acknowledges the financial support of the EU FP7 framework, through the Initial Training Network "Advanced Techniques in Computational Mechanics", grant agreement 238548. The second author would like to acknowledge the financial support received through "The Leverhulme Prize" awarded by The Leverhulme Trust, United Kingdom. The third and fourth authors acknowledge the financial support provided by the Sêr Cymru National Research Network for Advanced Engineering and Materials, United Kingdom.

\section{References}

[1] M. Aguirre, A. J. Gil, J. Bonet, A. Arranz-Carreño, A vertex centred finite volume Jameson-Schmidt-Turkel (JST) algorithm for a mixed conservation formulation in solid dynamics., Journal of Computational Physics 259 (2014) 672-699.

[2] G. Kluth, B. Després, Discretization of hyperelasticity on unstructured mesh with a cell-centered Lagrangian scheme, Journal of Computational Physics 229 (2010) 9092-9118.

[3] C. H. Lee, A. J. Gil, J. Bonet, Development of a cell centred upwind finite volume algorithm for a new conservation law formulation in structural dynamics, Computers and Structures 118 (2013) 13-38.

[4] F. Vilar, P.-H. Maire, R. Abgrall, A discontinuous galerkin discretization for solving the two-dimensional gas dynamics equations written under total lagrangian formulation on general unstructured grids, Journal of Computational Physics 276 (2014) 188 - 234.

[5] A. J. Gil, C. H. Lee, J. Bonet, M. Aguirre, A stabilised Petrov-Galerkin formulation for linear tetrahedral elements in compressible, nearly incompressible and truly incompressible fast dynamics, Computer Methods in Applied Mechanics and Engineering 276 (2014) 659-690. 
[6] N. P. Weatherill, O. Hassan, Efficient three-dimensional Delaunay triangulation with automatic point creation and imposed boundary constraints, International Journal for Numerical Methods in Engineering 37 (1994) 2005-2039.

[7] K. Miller, G. Joldes, D. Lance, A. Wittek, Total Lagrangian explicit dynamics finite element algorithm for computing soft tissue deformation, Communications in Numerical Methods in Engineering 23 (2007) 121134.

[8] C. Hesch, P. Betsch, A mortar method for energy-momentum conserving schemes in frictionless dynamic contact problems, International Journal for Numerical Methods in Engineering 77 (2009) 1468-1500.

[9] A. J. Gil, A. A. Carreño, J. Bonet, O. Hassan, The Immersed Structural Potential Method for haemodynamic applications, Journal of Computational Physics 229 (2011) 8613-8641.

[10] A. J. Gil, A. A. Carreño, J. Bonet, O. Hassan, An enhanced Immersed Structural Potential Method for fluid-structure interaction, Journal of Computational Physics 250 (2013) 178-205.

[11] C. Hesch, A. J. Gil, A. A. Carreño, J. Bonet, P. Betsch, A mortar approach for Fluid-Structure interaction problems: Immersed strategies for deformable and rigid bodies, Computer Methods in Applied Mechanics and Engineering 278 (2014) 853-882.

[12] C. Hesch, A. J. Gil, A. A. Carreño, J. Bonet, On continuum immersed strategies for Fluid-Structure Interaction, Computer Methods in Applied Mechanics and Engineering 247-248 (2012) 51-64.

[13] D. J. Benson, Computational methods in Lagrangian and Eulerian hydrocodes, Computer Methods in Applied Mechanics and Engineering 99 (1992) 235-394.

[14] T. J. R. Hughes, The Finite Element Method. Linear Static and Dynamic Finite Element Analysis, Dover Publications, 2000.

[15] D. J. Payen, K. J. Bathe, Improved stresses for the 4-node tetrahedral element, Computers and Structures 89 (2011) 1265-1273. 
[16] D. J. Payen, K. J. Bathe, A stress improvement procedure, Computers and Structures 112-113 (2012) 311-326.

[17] H. M. Hilber, T. J. R. Hughes, R. L. Taylor, Improved numerical dissipation for time integration algorithms in structural dynamics, Earthquake Engineering and Structural Dynamics 5 (1977) 283-292.

[18] W. L. Wood, M. Bossak, O. C. Zienkiewicz, An alpha modification of Newmark's method, International Journal for Numerical Methods in Engineering 15 (1980) 1562-1566.

[19] J. Chung, G. M. Hulbert, A time integration algorithm for structural dynamics with improved numerical dissipation: The generalized- $\alpha$ method, Journal of Applied Mechanics 60 (1993) 371-375.

[20] D. D. Adams, W. L. Wood, Comparison of Hilber-Hughes-Taylor and Bossak ' $\alpha$-methods' for the numerical integration of vibration equations, International Journal for Numerical Methods in Engineering 19 (1983) 765-771.

[21] Y. Onishi, K. Amaya, A locking-free selective smoothed finite element method using tetrahedral and triangular elements with adaptive mesh rezoning for large deformation problems, International Journal for Numerical Methods in Engineering 99 (2014) 354-371.

[22] F. M. A. Pires, E. A. de Souza Neto, J. L. de la Cuesta Padilla, An assessment of the average nodal volume formulation for the analysis of nearly incompressible solids under finite strains, Communications in Numerical Methods in Engineering 20 (2004) 569-583.

[23] T. Belytschko, J. S. Ong, W. K. Liu, J. M. Kennedy, Hourglass control in linear and nonlinear problems, Computer Methods in Applied Mechanics and Engineering 43 (1984) 251-276.

[24] D. P. Flanagan, T. Belytschko, A uniform strain hexahedron and quadrilateral with orthogonal hourglass control, International Journal for Numerical Methods in Engineering 17 (1981) 679-706.

[25] T. J. R. Hughes, Generalization of selective integration procedures to anisotropic and nonlinear media, International Journal for Numerical Methods in Engineering 15 (1980) 1413-1418. 
[26] J. Bonet, R. D. Wood, Nonlinear Continuum Mechanics for Finite Element Analysis, Cambridge University Press, 2nd edition, 2008.

[27] T. Elguedj, Y. Bazilevs, V. M. Calo, T. J. R. Hughes, $\bar{B}$ and $\bar{F}$ projection methods for nearly incompressible linear and nonlinear elasticity and plasticity using higher-order NURBS elements, Computer Methods in Applied Mechanics and Engineering 197 (2008) 2732-2762.

[28] A. J. Gil, P. D. Ledger, A coupled $h p$-finite element scheme for the solution of two-dimensional electrostrictive materials, International Journal for Numerical Methods in Engineering 91 (2012) 1158-1183.

[29] D. Jin, P. D. Ledger, A. J. Gil, An $h p$-fem framework for the simulation of electrostrictive and magnetostrictive materials, Computers and Structures 133 (2014) 131-148.

[30] E. J. Kubatko, C. Dawson, J. J. Westerink, Time step restrictions for Runge-Kutta discontinuous Galerkin methods on triangular grids, Journal of Computational Physics 227 (2008) 9697-9710.

[31] F. M. Capaldi, Continuum Mechanics. Constitutive Modeling of Structural and Biological Materials., Cambridge University Press, 2012.

[32] T. A. Laursen, Computational Contact and Impact Mechanics, volume 38, Springer, 2003.

[33] J. Bonet, A. J. Burton, A simple average nodal pressure tetrahedral element for incompressible and nearly incompressible dynamic explicit applications, Communications in Numerical Methods in Engineering 14 (1998) 437-449.

[34] S. K. Lahiri, J. Bonet, J. Peraire, A variationally consistent mesh adaptation method for triangular elements in explicit Lagrangian dynamics, International Journal for Numerical Methods in Engineering 82 (2010) 1073-1113.

[35] M. W. Gee, C. R. Dohrmann, S. W. Key, W. A. Wall, A uniform nodal strain tetrahedron with isochoric stabilization, International Journal for Numerical Methods in Engineering 78 (2009) 429-443. 
[36] M. A. Puso, J. Solberg, A stabilized nodally integrated tetrahedral, International Journal for Numerical Methods in Engineering 67 (2006) 841-867.

[37] C. Dohrmann, M. W. Heinstein, J. Jung, S. W. Key, W. R. Witkowski, Node-based uniform strain elements for three-node triangular and fournode tetrahedral meshes, International Journal for Numerical Methods in Engineering 47 (2000) 1549-1568.

[38] J. Bonet, H. Marriott, O. Hassan, Stability and comparison of different linear tetrahedral formulations for nearly incompressible explicit dynamic applications, International Journal for Numerical Methods in Engineering 50 (2001) 119-133.

[39] J. Bonet, H. Marriott, O. Hassan, An averaged nodal deformation gradient linear tetrahedral element for large strain explicit dynamic applications, Communications in Numerical Methods in Engineering 17 (2001) $551-561$.

[40] C. H. Lee, A. J. Gil, J. Bonet, Development of a stabilised PetrovGalerkin formulation for conservation laws in Lagrangian fast solid dynamics, Computer Methods in Applied Mechanics and Engineering 268 (2014) 40-64.

[41] I. A. Karim, C. H. Lee, A. J. Gil, J. Bonet, A two-step Taylor Galerkin formulation for fast dynamics, Engineering Computations 31 (2014) 366-387.

[42] N. Nguyen, J. Peraire, Hybridizable Discontinuous Galerkin Methods for partial differential equations in continuum mechanics, Journal of Computational Physics 231 (2012) 5955-5988.

[43] Y. Fryer, C. Bailey, M. Cross, C.-H. Lai, A control volume procedure for solving the elastic stress-strain equations on an unstructured mesh, Applied Mathematical Modelling 15 (1991) 639-645.

[44] C. Bailey, M. Cross, A finite volume procedure to solve elastic solid mechanics problems in three dimensions on an unstructured mesh, International Journal for Numerical Methods in Engineering 38 (1995) $1757-1776$. 
[45] A. Slone, C. Bailey, M. Cross, Dynamic solid mechanics using finite volume methods, Applied Mathematical Modelling 27 (2003) 69-87.

[46] G. A. Taylor, C. Bailey, M. Cross, A vertex-based finite volume method applied to non-linear material problems in computational solid mechanics, International Journal for Numerical Methods in Engineering 56 (2003) 507-529.

[47] N. Favrie, S. Gavrilyuk, Diffuse interface model for compressible fluid compressible elastic-plastic solid interaction, Journal of Computational Physics 231 (2012) 2695 - 2723.

[48] S. K. Sambasivan, M. J. Shashkov, D. E. Burton, A finite volume cell-centered Lagrangian hydrodynamics approach for solids in general unstructured grids, International Journal for Numerical Methods in Fluids 72 (2013) 770-810.

[49] J. A. Trangenstein, P. Colella, A higher-order Godunov method for modeling finite deformation in elastic-plastic solids, Communications on Pure and Applied Mathematics 44 (1991) 41-100.

[50] G. H. Miller, E. G. Puckett, A high-order Godunov method for multiple condensed phases, Journal of Computational Physics 128 (1996) 134164 .

[51] H. S. Tang, F. Sotiropoulos, A second-order Godunov method for wave problems in coupled solid-water-gas systems, Journal of Computational Physics 151 (1999) 790-815.

[52] B. P. Howell, G. J. Ball, A Free-Lagrange augmented Godunov method for the simulation of elastic-plastic solids, Journal of Computational Physics 175 (2002) 128-167.

[53] P.-H. Maire, R. Abgrall, J. Breil, R. Loubère, B. Rebourcet, A nominally second-order cell-centered Lagrangian scheme for simulating elasticplastic flows on two-dimensional unstructured grids, Journal of Computational Physics 235 (2013) 626-665.

[54] A. J. Gil, J. Bonet, J. Silla, O. Hassan, A discrete geometric conservation law (DGCL) for a cell vertex finite-volume algorithm on moving 
domains, International Journal for Numerical Methods in Biomedical Engineering 26 (2010) 770-779.

[55] G. Scovazzi, Lagrangian shock Hydrodynamics on tetrahedral meshes: A stable and accurate variational multiscale approach, Journal of Computational Physics 231 (2012) 8029-8069.

[56] G. Scovazzi, E. Love, M. Shashkov, Multi-scale Lagrangian shock Hydrodynamics on Q1/P0 finite elements: Theoretical framework and twodimensional computations, Computer Methods in Applied Mechanics and Engineering 197 (2008) 1056-1079.

[57] G. Scovazzi, E. Love, A generalized view on Galilean invariance in stabilized compressible flow computations, International Journal for Numerical Methods in Fluids 64 (2010) 1065-1083.

[58] J. Bonet, A. J. Gil, C. H. Lee, M. Aguirre, R. Ortigosa, A first order hyperbolic framework for large strain computational solid dynamics. part i: Total lagrangian isothermal elasticity, Computer Methods in Applied Mechanics and Engineering 283 (2015) 689 - 732.

[59] J. M. Ball, Convexity conditions and existence theorems in nonlinear elasticity, Archive for Rational Mechanics and Analysis 63 (1976) 337403.

[60] E. F. Toro, Riemann Solvers and Numerical Methods for Fluid Dynamics, Springer, 1999.

[61] O. Heuzé, General form of the Mie-Grüneisen equation of state, Comptes Rendus Mecanique 340 (2012) 679-687.

[62] C. M. Dafermos, Hyperbolic Conservation Laws in Continuum Physics, volume 44, Springer Netherlands, 2nd edition, 2009.

[63] J. Marsden, T. Hughes, Mathematical Foundations of Elasticity, Dover Publications, 1983.

[64] J. Bonet, A. J. Gil, R. Ortigosa, A computational framework for polyconvex large strain elasticity, Computer Methods in Applied Mechanics and Engineering (2014). Submitted. 
[65] M. A. Meyers, Dynamic Behavior of Materials, John Wiley and Sons, 1994.

[66] M. Arienti, E. Morano, J. Shepherd, Shock and detonation modeling with the Mie-Grüneisen equation of state, Technical Report, Graduate Aeronautical Laboratories, California Institute of Technology, 2004.

[67] S. Hiermaier, Structures under crash and impact: continuum mechanics, discretization and experimental characterization, Springer, 2008.

[68] J. Simo, Algorithms for static and dynamic multiplicative plasticity that preserve the classical return mapping schemes of the infinitesimal theory, Computer Methods in Applied Mechanics and Engineering 99 (1992) 61-112.

[69] A. J. Gil, Z. Zhang, O. Hassan, K. Morgan, Parallel multigrid detached eddy simulation algorithm for three-dimensional unsteady incompressible flows on unstructured meshes, Journal of Aerospace Engineering 19 (2006) 271-280.

[70] Z. Zhang, A. J. Gil, O. Hassan, K. Morgan, The simulation of 3D unsteady incompressible flows with moving boundaries on unstructured meshes, Computers and Fluids 37 (2008) 620-631.

[71] K. A. Sørensen, O. Hassan, K. Morgan, N. P. Weatherill, A multigrid accelerated hybrid unstructured mesh method for 3D compressible turbulent flow, Computational Mechanics 31 (2003) 101-114.

[72] A. Jameson, T. Baker, N. P. Weatherill, Calculation of inviscid transonic flow over a complete aircraft, AIAA 24th Aerospace Sciences Meeting (1986).

[73] D. J. Mavriplis, Unstructured grid techniques, Annual Review of Fluid Mechanics 29 (1997) 473-514.

[74] K. Morgan, J. Peraire, Unstructured grid finite-element methods for fluid mechanics, Reports on Progress in Physics 61 (1998) 569-638.

[75] T. J. Barth, D. C. Jespersen, The design and application of upwind schemes on unstructured meshes, AIAA 89-0366 (1989). 
[76] R. J. Leveque, Finite Volume Methods for Hyperbolic Problems, Cambridge University Press, 2004.

[77] P. L. Roe, Approximate Riemann solvers, parameter vectors, and difference schemes, Journal of Computational Physics 43 (1981) 357-372.

[78] S. Osher, F. Solomon, Upwind difference schemes for hyperbolic systems of conservation laws, Mathematics of Computation 38 (1982) pp. 339374 .

[79] R. Abedi, R. B. Haber, Riemann solutions and spacetime discontinuous Galerkin method for linear elastodynamic contact, Computer Methods in Applied Mechanics and Engineering 270 (2014) 150-177.

[80] M. Aftosmis, D. Gaitonde, T. S. Tavares, Behavior of linear reconstruction techniques on unstructured meshes, AIAA Journal 33 (1995) 2038-2049.

[81] H. Luo, J. Baum, R. Löhner, An improved Finite Volume scheme for compressible flows on unstructured grids, AIAA paper 95-0348 (1995).

[82] P. K. Sweby, High resolution schemes using flux limiters for hyperbolic conservation laws, SIAM Journal on Numerical Analysis 21 (1984) pp. 995-1011.

[83] T. Barth, D. Jespersen, The design and application of upwind schemes on unstructured meshes, in: Aerospace Sciences Meetings, American Institute of Aeronautics and Astronautics, 1989, pp. - .

[84] M. Torrilhon, Locally divergence-preserving upwind Finite Volume schemes for magnetohydrodynamic equations, SIAM Journal on Scientific Computing 26 (2005) 1166-1191.

[85] C. W. Shu, S. Osher, Efficient implementation of essentially nonoscillatory shock-capturing schemes, Journal of Computational Physics 77 (1988) 439-471.

[86] B. Cockburn, C. W. Shu, The Runge-Kutta Discontinuous Galerkin method for conservation laws V: Multidimensional systems, Journal of Computational Physics 141 (1998) 199-224. 
[87] R. Courant, K. Friedrichs, H. Lewy, On the partial difference equations of mathematical physics, Mathematische Annalem 100 (1928) 32-74.

[88] G. A. Sod, A survey of several finite difference methods for systems of nonlinear hyperbolic conservation laws, Journal of Computational Physics 27 (1978) 1-31.

[89] G. Scovazzi, M. Christon, T. Hughes, J. Shadid, Stabilized shock Hydrodynamics: I. A Lagrangian method, Computer Methods in Applied Mechanics and Engineering 196 (2007) 923-966.

[90] P. Woodward, P. Colella, The numerical simulation of two-dimensional fluid flow with strong shocks, Journal of Computational Physics 54 (1984) 115-173.

[91] W. Noh, Errors for calculations of strong shocks using an artificial viscosity and an artificial heat flux, Journal of Computational Physics 72 (1987) 78-120.

[92] S. Sambasivan, M. Shashkov, D. E. Burton, M. A. Christon, Mimetic theory for cell-centered Lagrangian finite volume formulation on general unstructured grids, Technical Report, Los Alamos National Laboratory, 2012 .

[93] J. Simo, N. Tarnow, The discrete energy-momentum method. Conserving algorithms for nonlinear elastodynamics, Zeitschrift für angewandte Mathematik und Physik ZAMP 43 (1992) 757-792.

[94] L. Noels, L. Stainier, J. P. Ponthot, An energy-momentum conserving algorithm for non-linear hypoelastic constitutive models, International Journal for Numerical Methods in Engineering 59 (2004) 83-114.

[95] S. Dong, BDF-like methods for nonlinear dynamic analysis, Journal of Computational Physics 229 (2010) 3019-3045.

[96] G. Scovazzi, Stabilized shock Hydrodynamics: II. Design and physical interpretation of the SUPG operator for Lagrangian computations, Computer Methods in Applied Mechanics and Engineering 196 (2007) 967-978. 
[97] G. Scovazzi, A discourse on Galilean invariance, SUPG stabilization and the Variational Multiscale framework, Computer Methods in Applied Mechanics and Engineering 196 (2007) 1108-1132.

[98] G. Scovazzi, J. Shadid, E. Love, W. Rider, A conservative nodal Variational Multiscale Method for Lagrangian shock Hydrodynamics, Computer Methods in Applied Mechanics and Engineering 199 (2010) 30593100 .

[99] G. H. Miller, An iterative Riemann solver for systems of hyperbolic conservation laws, with application to hyperelastic solid mechanics, Journal of Computational Physics 193 (2003) 198-225. 